UC-25

lesued: Augut 1978

\section{A Comparison of Initial Damage Rates Using Neutron and Electron Irradiations}

Joyce A. Roberts Goldstone

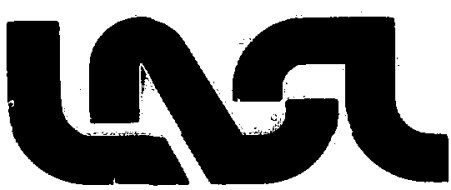

- NOTICE

This zeport was piepared as an ascount we wer the ponsoyed by the United States Gevernment. Nethen to Unted Sistes no: the United Stales Departiment of Energy, nor any of their employres, not any of thet contracturs, subrontractors, or theil calpsioyes any legal any warranty, exorsiblity for the accuracy, completeness liablity or tesponsibing formation, apparatus, product or ot usefulness of any in presents that its use woutd no process disclosed. or represents 
CONTENTS

List of Figures . . . . . . . . . . . . . . . . . . vi List of Tables . . . . . . . . . . . . . . . viii Abstract . . . . . . . . . . . . . . . . . ix

I Introduction . . . . . . . . . . . . . . . . 1

A. Defect Production . . . . . . . . . . . . 1

B. Defect Type and Size Experiments . . . . . . . . 17

C. Electrical Resistivity Experiments . . . . . . 23

D. Internal Friction Theory . . . . . . . . 31

E. Internal Friction Measurements . . . . . . . 35

F. Present Experiment . . . . . . . . . . 39

II Experimental Procedures .............. 43

A. Sample Preparation .. . . . . . . . . 43

B. Electronics ................ 47

C. Calculation of Measured Parameters . . . . . . 53

D. Measurement of the Strain Amplitude . . . . . . 54

E. Irradiations . . . . . . . . . . . . 56

F. Defect Production . . . . . . . . . . 65

III Experimental Results and Analysis . . . . . . . . 69

A. Introduction ................ 69

B. Flux Dependence in Copper ........... 75

C. Temperature Dependence in Copper . . . . . . . . 80

D. Neutron Energy Dependence in Copper . . . . . . . 81

E. Gold Data . . . . . . . . . . . . 92 
A. Flux Dependence in Copper . . . . . . . . . . . 99

B. Temperature Dependence in Copper . . . . . . . . 101

c. Free Interstitial Production Cross Section.... . 103

D. Comparison to Computer Models... . . . . . 115

V Conclusions . . . . . . . . . . . . . . . . 118

A. Summary of Results . . . . . . . . . . . . 118

B. Extensions of the Present Experiment . . . . . . 120 Acknowledgments . . . . . . . . . . . . . . . . 123 References . . . . . . . . . . . . . . . . . 124 Appendix . . . . . . . . . . . . . . . . . . . 130 


\section{FIGURES}

Figure 1: Recoil Energy Spectrum in Copper due to

$2 \mathrm{MeV}$ Neutrons . . . . . . . . . . . . 6

Figure 2: Recoil Energy Spectrum in Copper due to

$14 \mathrm{MeV}$ Neutrons ........... . 7

Figure 3: Neutron Damage Cross Section in Copper

from 1 to $24 \mathrm{MeV}$. . . . . . . . . . . . 13

Figure 4: Displacement Cross Section from 1 to 24

$\mathrm{MeV}$ in Copper............ . . 14

Figure 5: Decrement and Modulus Defect in Simpson-

Sosin Model . . . . . . . . . . . . 34

Figure 6: Sample Configuration . . . . . . . . . . . . 46

Figure 7: Sample in FM Oscillator Circuit . . . . . . . 48

Figure 8: Flow Chart of the Electronics . . . . . . . 50

Figure 9: Flow Chart of the Temperature Controller . . . . 52

Figure 10: Strain Dependence of the Damping . . . . . . . 57

Figure 11: Van de Graaff Experimenta1 Set-up . . . . . . . 61

Figure 12: Raw Data for $0.5 \mathrm{MeV}$ Electron Irradiation

of Copper . . . . . . . . . . . . . 73

Figure 13: Reduced Data for Raw Data of Figure 12 . . . . 74

Figure 14: $\mathrm{N}_{\mathrm{dy}}$ Versus Time for Electron Flux

Dependence Runs in Copper . . . . . . . . 77 
Figure 15: $\mathrm{N}_{\mathrm{dy}}$ Versus Time for Neutron Flux

Dependence Runs in Copper. . . . . . . . . 78

Figure 16: Flux Dependence of the Initial Pinning Rate . . . 79

Figure 17: $\mathrm{N}_{\mathrm{dy}}$ Versus Time for Electron Temperature

Depend ance Runs in Copper . . . . . . . . . . 82

Figure 18: $\mathrm{N}_{\mathrm{dy}}$ Vercus Time for Neutron Temperature

Dependence Runs in Copper . . . . . . . . . 83

Figure 19: Temperature Dependence of the Initial

- Pinning Rate.. . . . . . . . . . . . . 84

Figure 20: Raw Data for $6.0 \mathrm{MeV}$ Neutron Irradiation

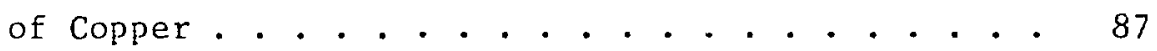

Figure 21: Reduced Data for Raw Data of Figure 20 . . . . 88

Figure 22: N $\mathrm{N}_{\mathrm{dy}}$ Versus Time for Neutron Energy

Dependence Runs in Copper . . . . . . . . . 89

Figure 23: Relative Pinning Versus Neutron Energy for

Copper with the Three Displacement Mode1s . . . . 91

Figure 24: Raw Data for 2.0 MeV Electron Irradiation

of Gold . . . . . . . . . . . . . . . 95

Figure 25: Reduced Data for Raw Data of Figure 24 . . . . . . 96

Figure 26: Raw Data for $14.3 \mathrm{MeV}$ Neutron Irradiation

of Gold.................... . . 97

Figure 27: Reduced Data Eor Raw Data of Figure 26 . . . . . 98

Figure 28: Schematic Diagram of Van de Graaff

Irradiation Geometry . . . . . . . . . . . 131 
Table 1: Recoil-Energy and Damage-Energy Spectra . . . . . 16

Tab1r 2: Typical Analyses of Copper and Gold Rod . . . . . . 44

Table 3: Experimental Neutron Energies and Fluxes . . . . . 63

Table 4: 0.5 MeV Electron Irradiations of Copper . . . . . 71

Table 5: Neutron Irradiations of Copper . . . . . . . 72

Table 6: Pinning Points per Neutron in Copper . . . . . . . 90

Table 7: Electron and Neutron Irradiations of Gold . . . . . 93

Table 8: Free Interstitial. Production Cross Section . . . . 106

Table 9: Free Interstitials per Cascade . . . . . . . . 109 


\author{
Abstract of the Dissertation \\ A Comparison of Initial Damage Rates Using \\ Neutron and Electron Irradiations \\ by \\ Joyce A. Roberts Goldstone* \\ Doctor of Philosophy \\ in \\ Materials Science \\ State University of New York at Stony Brook
}

1978

The purpose of this experiment was twofold: (1) The number of interstitials that pin dislocations was studied as a function of neutron energy. (2) By comparison with electron irradiations on the sample, a correlation between the predicted and measured numbers of defects was found.

Al1 irradiations were performed on the same high purity copper sample. The sample was machined in the form of a cantilever beam with a flexural resonant frequency of $770 \mathrm{~Hz}$. Changes in Young's modulus at constant strain amplitude were monitored continuously through changes in the resonant frequency of the sample. These changes in the modulus can be related to the number of pinning points added to dislocation lines, which are in turn related to the number of free interstitials produced. *Thesis accepted under the name Joyce Ann Roberts. 
The initial pinning rate (number of pinning points added per unit time near the onset of irradiation) was found to depend on the flux to the $0.87 \pm 0.02$ power in both neutron and electron irradiations. Temperature dependence studies from 310 to $390 \mathrm{~K}$ also showed the same dependence for neutron and electron irradiations with an activation enthalpy of $0.17 \pm 0.02 \mathrm{eV}$. Thus the free interstitial was the pinning defect in both cases.

Neutron energy dependence experiments were done from 2 to $24 \mathrm{MeV}$ on the copper sample and at $14 \mathrm{MeV}$ on a gold sample. By equating pinning rates from electron and neutron irradiations and using the free interstitial production rate obtained from electron irradiations, an estimate of the free interstitial production cross section for neutrons of 2 to $24 \mathrm{MeV}$ was made. These values were compared with several theories which predict the number of Frenkel pairs produced by neutron irradiations. Comparison with the simplest of these models (NRT model) showed good agreement with the trend of the energy. dependence of the pinning rate. Additional comparison showed that only $1 \%$ or less of the predicted number of interstitials produced survive as free interstitials. 


\section{Introduction}

\section{A. Defect Production}

A fundamental problem ir radiation damage by high energy neutrons (particularly energies around $14 \mathrm{MeV}$ ) is the relation between the energy of the incoming neutron and the number of point defects produced in the subsequent cascade. For metals, the major defect production mechanism is the displacement of an atom from its original lattice site a sufficient distance to form a stable Frenkel pair. Low energy or light particle (e.g., eler-trons) irradiations produce simple defect structures mainly composed of isolated vacancies and interstitials. Higher energy irradiations with more massive particles (neutrons, protons, ions) create more complex defect structures, displacement cascades, comprised of isolated Frenkel pairs, vacancy enriched regions (depleted zones), and areas of high interstitiai concentration. The variation from simple to complex defect structures corresponds to the increase in initial energy of the recoiling atom. If one can describe the distribution of recoil energies for a given irradiation, the problem reduces to determining the damage composition for a given recoil energy.

A qualitative assessment of damage structure due to ircreasing recoil energy(T) can be made relative to the threshold energy $\left(E_{\mathrm{d}} \cong 2.5 \mathrm{eV}\right.$ ) for stable Frenkel pair production. The threshold energy as used here is a value averaged over 
crystallographic directions and applicable to polycrystalline samples. However, not all the energy $T$ is avallable for defect production. Some of this energy goes into inelastic collisions, exclting electrons or lonizing other lattce atoms. When this portion of $T$ is subtracted, the resultant energy, $T_{\text {dam }}$ is referred to as the damage energy. At low values of damage energy, $T_{\text {dam }} \leq E_{d}$, damage can still result around defects which lower the displacement energy or along crystallographic directions which have lower threshold energies than the average value. These are referred to as subthreshold events. At $T_{\text {dam }} \geq E_{d}$, the production of one or more stable Frenkel pair is probable. As $T_{\text {dam }}$ increases so that $T_{\text {dam }}>E_{d}$, multiple displacements occur creating several Frenkel pairs per initial recoiling atom. Eventually as $T_{\text {dam }} \gg E_{d}$, cascades composed of thousands of displaced atoms develop. Given high enough values of $\mathrm{T}_{\text {dam }}$, secondary cascades result from high energy transfer events of the primary knock-on atom with other lattice atoms. In some irradiation spectra, recoil events can span the range from $T_{\text {dam }}\left\langle E_{d}\right.$ to $T_{\text {dam }} \gg E_{d}$ producing a variety of damage structures in the material.

Electron irradiations provide a particularly simple defect structure. The maximum energy transferred by a relativistic electron of energy $E_{e}$ is:

$$
T_{\max }=2 E \mathrm{e}^{\left(E_{\mathrm{e}}+2 m c^{2}\right) / M c^{2}}
$$


where $m$ is the mass of the electron $\left(5.49 \times 1 p^{-4}\right.$ amu), c is the speed of $11 \mathrm{ght}\left(c^{2}=931.5 \mathrm{MeV} / \mathrm{amu}\right)$, and $\mathrm{H}$ is the mass of the tary get atom. For an electron energy of $1.0 \mathrm{MeV}$, the maximum recoli energy of a copper atom $(\epsilon 3.54 \mathrm{amu})$ is $68 \mathrm{eV}$. If the displacement energy is between 20 and $25 \mathrm{eV}$ as suggested for copper, the number of Frenkel pairs produced per recoll will be no more than theer The recoll energies are distributed below $\mathrm{T}$ ax according to the Mott differential scattering cross section (Mc48), thus low energy recoils are more probable than those near $T$ max ${ }_{0}$ Tables of cross sections for atomic displacements by fast electrons have been computed by oen $(0 \mathrm{e} 73)$

Thermal neutron irradiations also provide low entres recoil spectra in materials by capture of the neutron ând subsequent emission of gama rays. "The kinetic energy of the recoiling atom for an emitted gamma ray energy $\mathrm{E} \mathrm{\gamma}$ is:

$$
\mathrm{T}_{\gamma}=\mathrm{E}_{\gamma}^{2} / 2 \mathrm{Mc}^{2}
$$

For most elements, the total emitted gamma ray engy per thermal

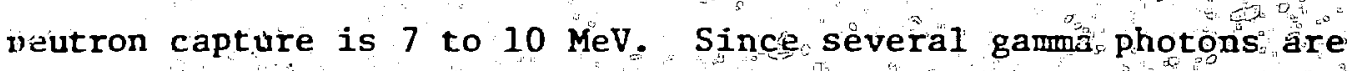
emitted per capture, the actual recoil energy of the atom depends on the time- and angular-distribution of the photons. To calculate a mean recoil energy, the assumptions are made that the emission of gamma rays is isotropic and uncorrelated. In copper, for example, the mean recoil energy is $374 \mathrm{eV}$ (Co62), so the number of defects per thermal neutron capture is roughly 7 . 
Irradiation by lons lnvolves higher recoll energies than electrons or thermal neutrons. For example, a $1 \mathrm{MeV}$ proton can cause copper atoms to recoll with energies of $62 \mathrm{keV}$ or roughly 1000 times the maximum recoil energy for a 1 MeV electron. The maximum energy transferred by an ion is:

$$
T_{\max }=4 M m E_{p} /(M+m)^{2}
$$

where $m$ is the mass of the irradiating particle and $M$ is the mass of the target atom." Over a wide range of bombardment energies, the scattering of fast ions obeys the Rutherford differential scattering cross section. The important feature of this scattering law is the dependence on $\mathrm{T}^{-2}$ producing doninately low energy recoils:

$$
\mathrm{d} \sigma / \mathrm{dT} \propto \mathrm{T}^{-2} .
$$

This coulomb cross section will not de strictly valid when the projectile is of sufficient energy to penetrate the nucleus and corrections for nuclear forces must be made. At high energies, inelastic nuclear processes can also occur. Thus the probability of some recoil energies will be enhanced and others reduced relative to the Rutherford scattering law. Ions may also behave as if they were displaced atoms rather than primary particles if their incident energy is sufficiently small (below $140 \mathrm{keV}$ for a copper ion in copper). The complexity of the defect structure in ion irradiations depends on the incident particle's mass and energy. 
With fast neutron 1rradiation, the coulomb interaction is absent and direct nuclear collisions give rise to atomic displacement6. Elastic collisions transfer a maximum energy given by equation $\mathrm{I}-3$, so a $14 \mathrm{MeV}$ neutron can impart a kinetic energy of $850 \mathrm{keV}$ to a copper.atom. In addition to elastic collisions, nonelast1c neutron processes also take place. In these processes, the neutron is absorbed by the nucleus and ralses it to an excited level. The nucleus is de-excited by emission of at least one particle. Examples of such reactions are $\left(n, n^{\prime}\right),(n, 2 n),(n, 3 n)$, $\left(n, n^{\prime} p\right),(n, p)$, and $(n, \alpha)$. Inelastic neutron scattering refers to those nonelastic events in which a neutron is reemitted and the decay of the excited nucleus is by gamma or particle emission $\left[\left(n, n^{\prime} \gamma\right),\left(n, n^{\prime} p\right)\right]$. Cross section information is available from the ENDF/B documentation $\left(\mathrm{O}_{2} 73\right)$.

The span of interesting recoil energies for primary knock-on atoms (PKAs) due to the above neutron reactions ranges from $E_{d}$ to $\sim 1 \mathrm{MeV}$. The primary-atom recoil spectrum is a function of the irradiating neutron energy. In Figures 1 and 2, exarples of the calculated recoil spectrum for copper irradiated by 2 and $14 \mathrm{MeV}$ neutrons are given. The lower energy part of the spectrum (below $20 \mathrm{keV}$ ) is almost identical in both figures. The high energy hump in the $2 \mathrm{MeV}$ spectrum is due to anisotropic elastic scattering, which produces high energy recoils because the angular distribution is forward peaked. The higher energy hump in the 14 


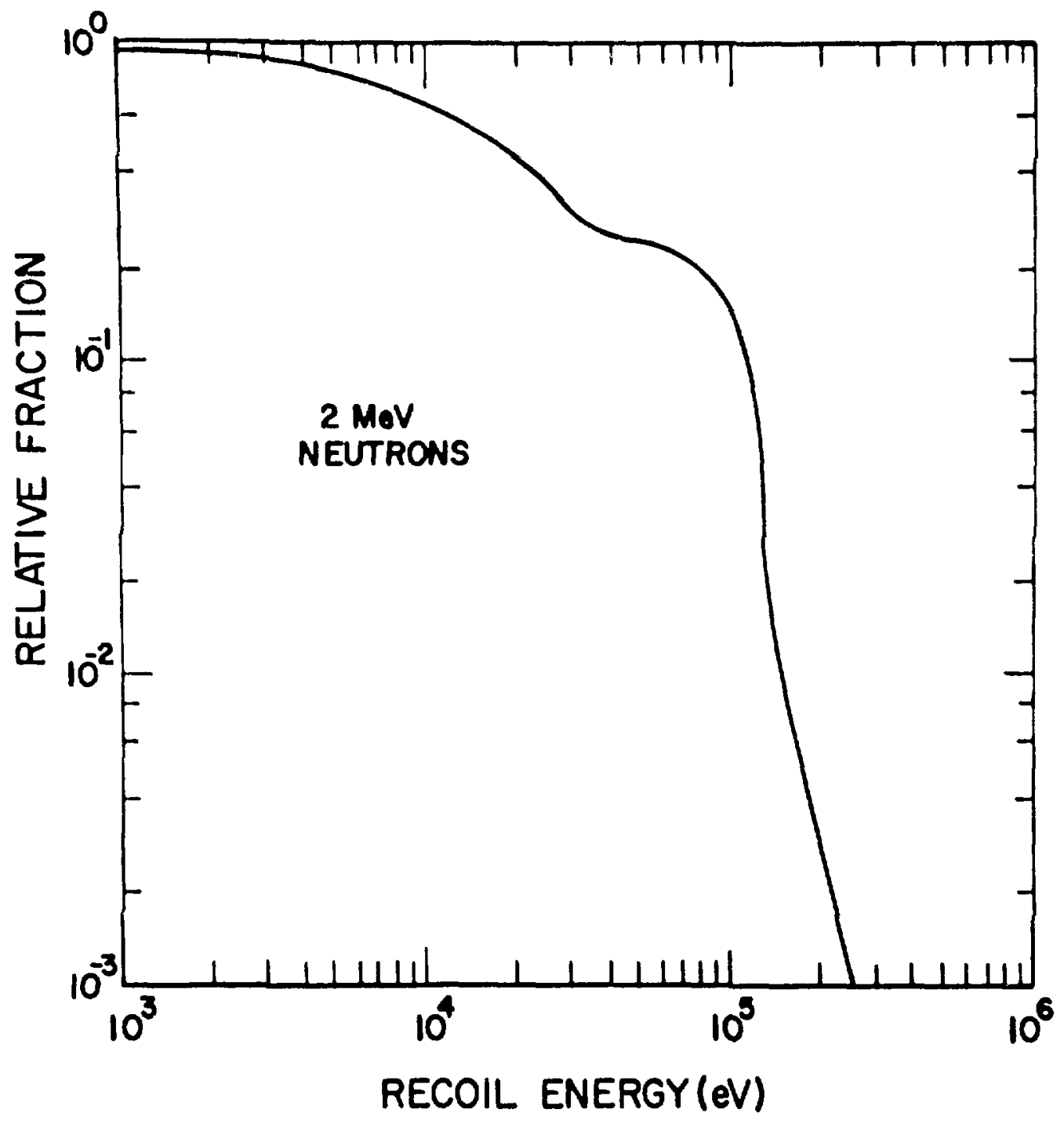

Figure 1: This figure shows the PKA recoil energy spectrum in copper irradiated with $2 \mathrm{MeV}$ neutrons. The relative fraction is given per unit energy and normalized to low energy recoils. 


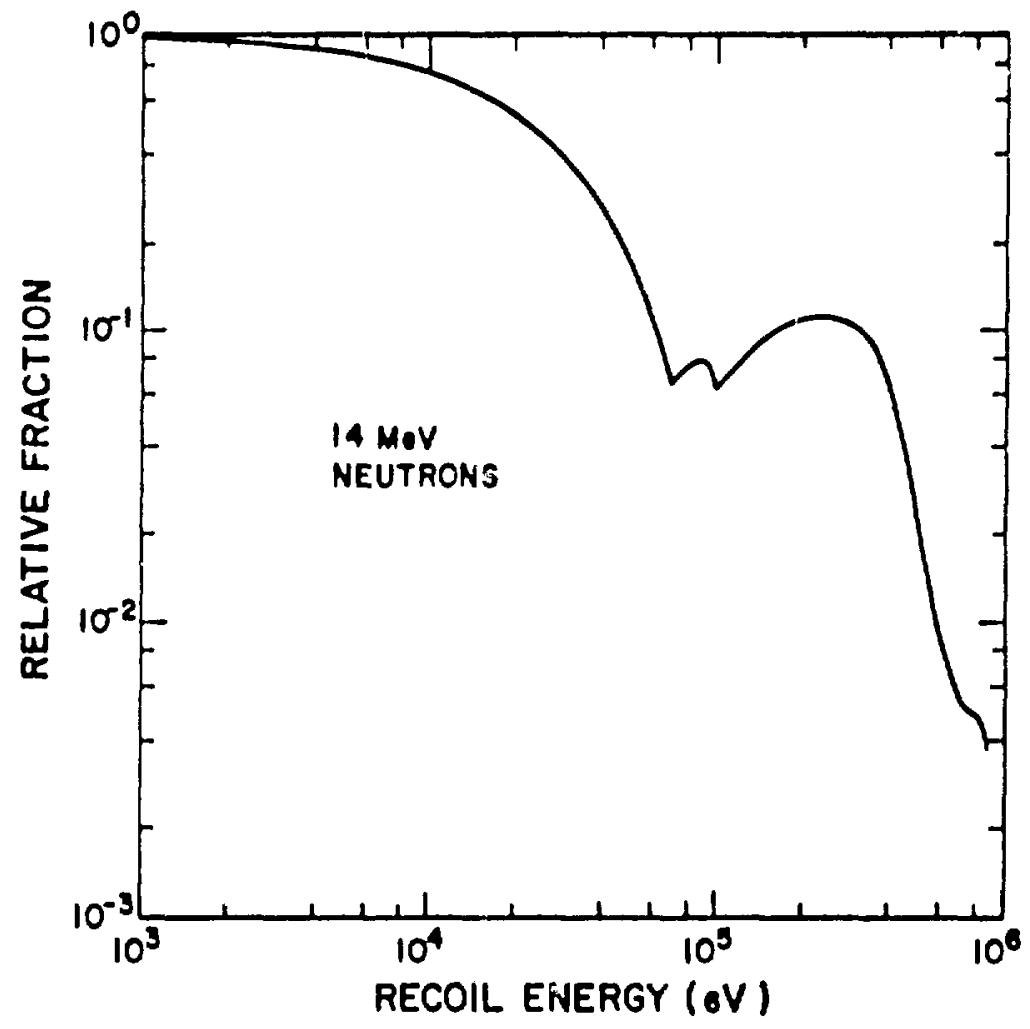

Figure 2: This figure shows the PKA recoil energy spectrum in copper irradiated with $14 \mathrm{MeV}$ neutrons. The relative fraction is given per unit energy and normalized to low energy recoils. 
$\mathrm{MeV}$ spectrum is due to nonelastic scattering, while the smaller hump is also due to antsotropic elastic scattering. The position of the hump moves to h1gher recoll energy as the neutron energy is increased. It w1ll be shown later that since the most damage comes from the higher energy recolls, the shift of this hump is more important than at first appears. The product of the probability that a neutron of energy $E_{n}$ will create a PKA energy between $T$ and $T+d T, K_{1}\left(E_{n}, T\right)$, via the $i-t h$ scattering mechanism and the cross section for the 1 -th scattering mechanism, $\sigma_{i}\left(E_{n}\right)$, is the recoil probability cross section. This cross section is the basis for computer calculations in radiation damage (Pa74, Do 76, Ga76, Fu76).

The relationship between the primary recoil spectrum and the observed property changes upon irradiation is elusive. The initial energy $T$ of a PKA is dissipated in collisions with other lattice atoms until it comes to rest at, typically, an interstitial site some distance from its corresponding vacancy. As previously discussed, the recoil energy is divided into two components: one component is the total electronic excitation energy loss and the other, $\mathrm{T}_{\mathrm{dam}}$, is the damage energy available to cause atom displacements. Commonly, LSS (Li63) theory is used to calculate the proportionment between these two processes. The result is approximated in simple analytic form by Robinson (Ro70):

$$
\begin{aligned}
T_{\text {dam }} & =T /\left[1+k_{L} f(n)\right] \\
k_{L} & =0.133745 z^{2 / 3} \mathrm{~A}^{-1 / 2}
\end{aligned}
$$




$$
\begin{aligned}
\eta & =T E_{1}^{-1} \\
E_{L} & =86.9312^{7 / 3} \\
f(\eta) & =\eta+0.40244 \eta^{3 / 4}+3.4008 \eta^{1 / 6} .
\end{aligned}
$$

Thus the damage energy depends on the atomic number, $Z$, and the mass, A, of the irradiated material. For recoil energies of 100 and $400 \mathrm{keV}$, the damage energles are 63.5 and $203.0 \mathrm{keV}$ in copper and 73.1 and $267.8 \mathrm{keV}$ in gold. As the recoil energy increases, the percentage of energy creating damage decreases.

Once the recolling atom dissipates all its energy, the condition for stability of the created Frenkel pairs determines the survival of defects. One model requires only that the atom be given an energy greater than a threshold energy, $E_{d}$, as described earlier. Another model defines a vacancy capture radius, $r_{v}$, within which the interstitial atom will be annihilated even at $0 \mathrm{~K}$. It is possible to consider $r_{v}$ as the range of a particle with energy $E_{d}$. The recombination model using $r_{v}$ is normally used in computer simulations where atoms are followed to very low energies. The first model generates a particularly simple form for the dependence of Frenkel pair production on the damage energy. This relation is called the damage energy, or modified Kinchin-Pease (Ki55), or the NRT (No75) mode1:

$$
g(T)=K T_{\text {dam }} / 2 E_{d} ; T_{d a m}>2 E_{d} / K
$$

where $k=0.8$ is the displacement efficiency and $g(T)$ is the 
recoll-dependent generallzed damage function. The displacement efflciency would be one for a hard-sphere potential as first suggested by KInchin and Pease, but Robinson (Ro70) found for a more realistic interatomic potential the effictency is less than one. This simple analytic model is accepted as the exposure index for partjcle irradiations.

The second criterion for stable Frenkel pair production has been applied in several computer simulations of defect production (Be66,Ro74). By using interatomic potentials and following all atoms in a particular cascade, cascade structure can be determined. Since the nascent cascade occurs in a few hundredths of a picosecond, this can be separated from the decay of unstable Frenkel pairs occurring in a time span of several picoseconds. Choices of how to follow cascading atoms and what position they take at rest produce the major difference between the Beeler(Be66) and Rohinson-Torrens (Ro74) models. The Beeler model, developed from cascade simulations up to damage energies of $20 \mathrm{keV}$, gives a logarithmic dependence of the defect production on the PKA recoil energy:

$$
g(T)=A\left[I-B \ln \left(T_{\text {dam }}\right)\right] T_{\text {dam }}
$$

where $A=9.65 \mathrm{keV}^{-1}$ and $B=0.0315$ for copper. The vacancy capture model of Robinson and Torrens, limited to damage energies of $9 \mathrm{keV}$ or less, yields a much stronger dependence on the damage energy: 


$$
g(T)=T_{d a m} /\left(\alpha+B T_{d a m}\right)
$$

where $\alpha=0.058 \mathrm{keV}$ and $\beta=1.25 \mathrm{keV}^{-1}$ for copper and both $\alpha$ and $B$ are dependent on the vacancy capture radius $r_{v}$. Adaltionally, Robinson and Torrens fit the1r simulations to a logarithmic function and find for copper $A=16.65 \mathrm{keV}^{-1}$ and $B=0.0401$. In the future, these models will be referred to as NRT (equation I-6), RT-b (equation I-7 with Robinson-Torrens values for $A$ and $B$ ), and RT-3 (equation I-8).

These displacement models give total numbers of defects produced per primary knock-on atom. Some annealing takes place in the cascade, other than recombination of an interstitial with its original vacancy. Doran, Simons, and McElroy (Do75), using the cascade structure input from the Robinson-Torrens simulation code, compute the number of residual defect pairs and the number of motile vacancies which escape the cascade. Interaction ire only considered within the cascade, and the effects of other cascades as sinks are ignored, and initial recoil energies are considered up to $100 \mathrm{keV}$. Since we are interested in the number of mobile defects in our experiments, only that energy dependence will be given. The number of mobile vacancies is:

$$
\begin{aligned}
g(T) & =0.519\left(T^{2}+42.52 T\right)^{I / 2} \quad 0.50 \leq T \leq 100 \mathrm{kel} \\
& =3.261 \mathrm{~T}+0.8696 \quad 0.04 \leq T \leq 0.50 \mathrm{keV}
\end{aligned}
$$

These equations can be extrapolated above $100 \mathrm{kel}$ by assuming linearity with damage energy, $T_{\text {dam }}$, and matching the value and 
slope at $100 \mathrm{keV}$. The model is referred to as the residual defect model and denoted by RDM.

By combining the total recoll probability cross sections, $\sum_{1} \pi_{1}\left(E_{n}\right) K_{1}\left(E_{n}, T\right)$, and one of the displacement models, and integrating over the recoll energies and the neutron spectrum, the displacement cross section can be caiculated

$$
\sigma_{d}=\int_{0}^{\infty} \sigma\left(E_{n}\right) \phi\left(E_{n}\right) \int_{0}^{\infty} k\left(E_{n}, T\right) g(T) d E d T
$$

where $\phi\left(E_{n}\right)$ is the neutron spectrum and $g(T)$ is given by one of the above displacement models. The dpa (displacements per atom) cross section is defined for the NRT model only and is equal to the displacement cross section for this model. If a monoenergetic neutron source is used, we need the displacement cross section at only one energy, $\sigma_{d}\left(E_{n}\right)$. The damage energy cross section for a monoenergetic source is defined by:

$$
\sigma_{D E}\left(E_{n}\right)=\int_{n}^{\infty} \sigma\left(E_{n}\right) K\left(E_{\Pi}, T\right) T_{d a m} d T
$$

In Figure 3, the damage energy cross section in copper is shown from 1 to $24 \mathrm{MeV}$. It can be seen that nonelastic collisions contribute the major portion of the total cross section at higher energies. The major contribulions to the total nonelastic cross section are shown by the dashed lines. The displacement cross sections for the four models from 1 to $24 \mathrm{MeV}$ are shown in Figure 4. The first three models, NRT, RT-b, and RT-3, presented are 


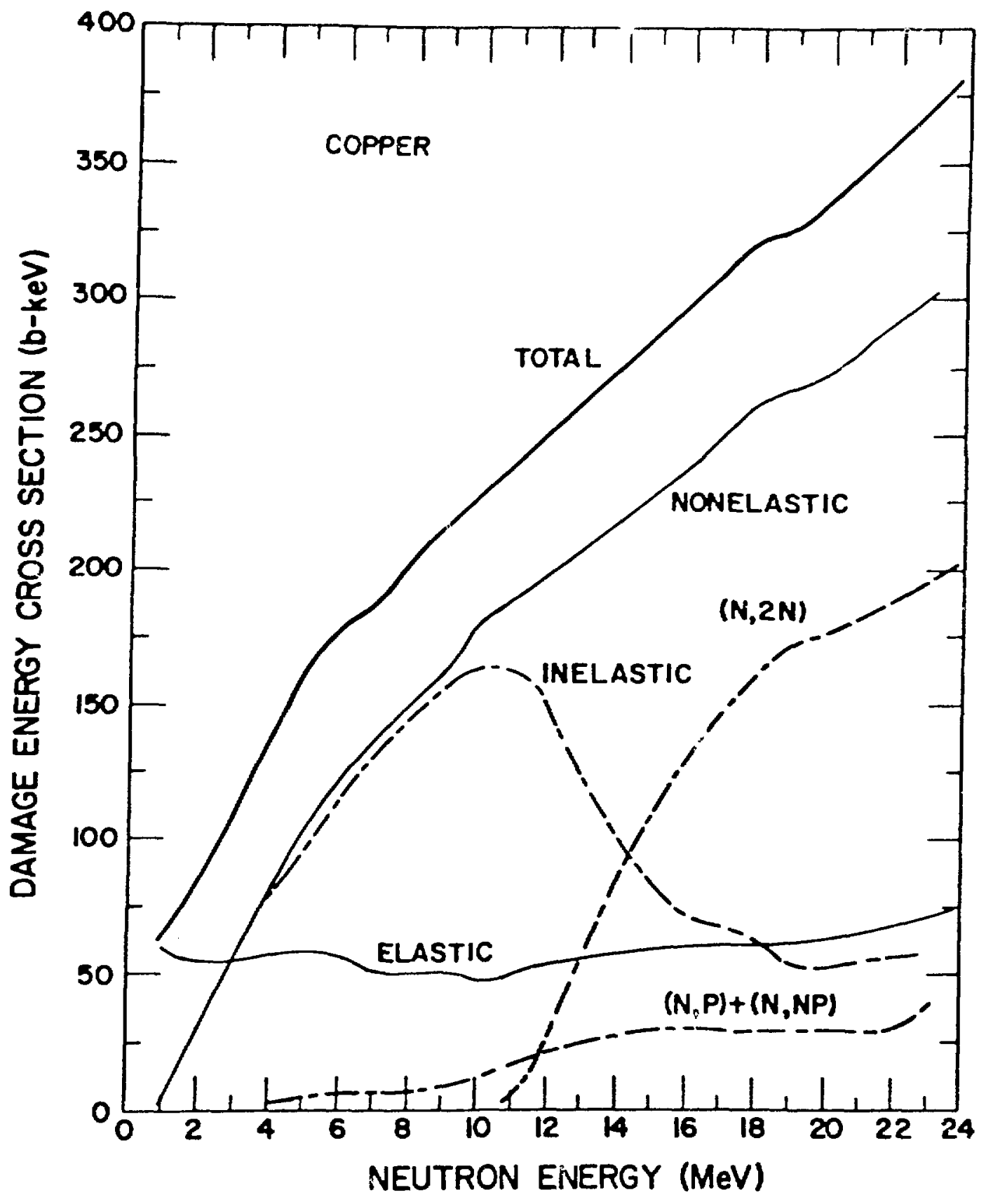

Figure 3: The damage energy cross section for copper from 1 to $24 \mathrm{MeV}$. The nonelastic contribution is the major part at higher energies. The dashed lines show the principal contributions to the total nonelastic cross section. 


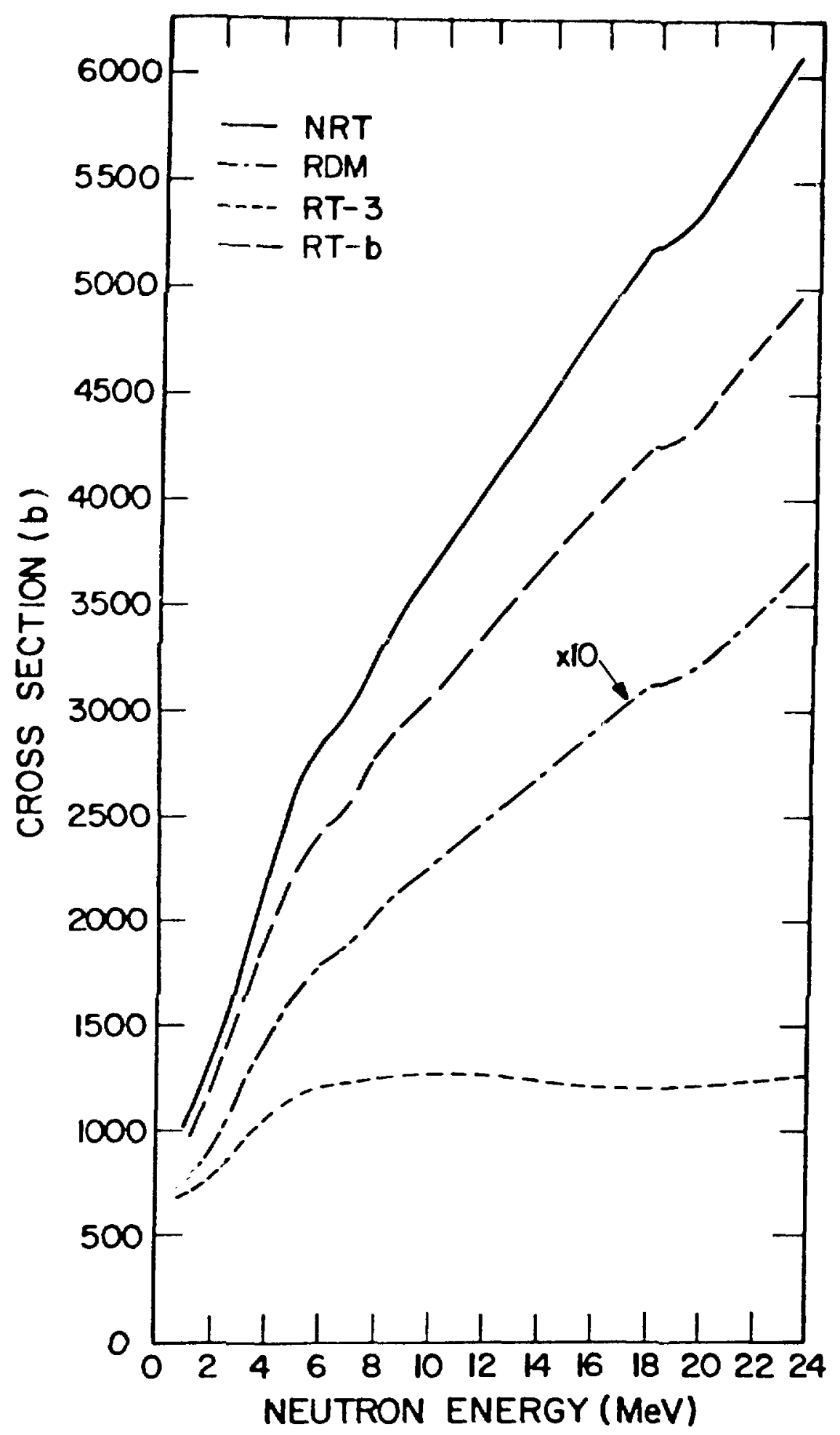

Figure 4: The displacement cross sections for the three displacement models are shown from 1 to $24 \mathrm{MeV}$. Note that the RDM cross sections have been multiplied by 10 so as to be clear. 
essentfally identical at low energies (e.g. recoll energies less than $20 \mathrm{keV}$ ) and only the high energy projections are different. The residual defect model cross section is less than $10 \%$ of the NRT cross section at $14 \mathrm{MeV}$. Technically, these models are calculated for low energy recolls typically $20 \mathrm{keV}$ or less, due to computer limitations. Extension of these models into the several hundred keV range may be unwarranted. It is very obvious that the RT-b and RT-3 models have very different high energy projections, while both equally well fit the Robinson-Torrens low energy computer simulations.

By studying the neutron energy dependence of a change in a physical property (resistivity, elastic modulus) or the number of defect clusters, a relative comparison can be made with the calculated displacement cross sections. The difference between the NRT and RT-b models is small particularly if one looks at the slope of $\sigma_{d}$ rather than absolute values. However, the separation between the energy depencience of the RT-3 model and the NRT and RT-b models should allow an experimental distinction to be made. By limiting the range of integration of equation $I-10$ to a particular recoil energy interval, the damage produced per recoil energy interval can be examined in relation to the ncrmalized recoij energy spectrum. In Table 1, the recoil energy and damage energy spectra are listed. $P(T)$ is the percentage of recoils within the energy interval and $\mathrm{T}_{\mathrm{dam}}$ is the percentage of the 
TABLE 1

\section{RECOIL-ENERGY AND DAMAGE-ENERGY SPECTRA}

\begin{tabular}{c}
$\begin{array}{c}\text { Recoil } \\
\text { Energy } \\
\text { Interval } \\
\text { (keV) }\end{array}$ \\
\hline $0-0.1$ \\
$0.1-1$ \\
$1-5$ \\
$5-10$ \\
$10-50$ \\
$50-100$ \\
$100-200$ \\
$200-400$ \\
$400-600$ \\
$600-T_{\max }$
\end{tabular}

\begin{tabular}{ll}
$\begin{array}{c}\mathrm{P}(\mathrm{T}) \\
(\%)\end{array}$ & $\frac{2 \mathrm{MeV}}{\mathrm{T}_{\text {dam }}}$ \\
\cline { 1 - 1 } 0.3 & $\frac{0}{\%)}$
\end{tabular}

\begin{tabular}{ll}
$\begin{array}{l}14 \mathrm{MeV} \\
\mathrm{P}(\mathrm{T}) \\
(\%)\end{array}$ & $\begin{array}{l}\mathrm{T}_{\text {dam }} \\
(\%)\end{array}$ \\
\hline 0.2 & 0.0
\end{tabular}

2.5

0.0

1.4

0.0

9.9

0.8

5.7

0.1

10.1

2.0

6.3

0.4

39.5

25.6

25.0

4.7

7.1

3.5

28.0

46.6

13.1

13.4

9.3

24.1

30.4

49.3

0.4

0.9

8.4

20.9

0.0

0.0

2.5

7.6 
total damage energy cross section for the same recoil energy Interval. It can be seen that low energy recolls do not contribute much to the damage. At $2 \mathrm{MeV}$, almost $50 \%$ of the damage energy cross section comes from recoils between 50 and $100 \mathrm{keV}$ where the small hump is present in Figure 1. Similarly for $14 \mathrm{MeV}$ neutrons, $50 \%$ of the damage cross section is generated by recoils between 200 and $400 \mathrm{keV}$, again in the region of the hump in Figure 2. Thus the higher energy recoils are more important in determining damage than the low energy recoils.

\section{B. Defect Type and Size Experiments}

Direct observation of the damage state is possible by using transmission electron microscopy (TEM). The number of vacancies in a particular cascade has to be sufficiently large to form a collapsed depleted zone of diameter greater than $20 \AA$ to be observed by TEM techniques. An uncollapsed depleted zone does not produce enough strain to be visible in the TEM. To study indivi-a dual cascades, the irradiation dose must be low enough so that intercascade reactions do not occur. Additionally, problems may arise from the loss of defects to the surface through image forces.

Self-ion bombardments of materials have been conducted in attempts to study the damage as a function of recoil energy (Th69, Th69a, Me74). Besides studying the defect size and type distributions, these experiments yield an estimate of the recoil energy 
necessary to consistently produce a vielble cascade and to produce vistble subcascades. Vacancy loops were the dominant defect cluster observed in self-ion irradiations of both copper and gold. For gold, Merkle (Me74) has fourd for recoll energies (self-ion bombardment energies) above $50 \mathrm{keV}$ that a visible cascade was always produced. About $T=100 \mathrm{keV}$, the possibility for subcluster formation rose to $50 \%$ in gold and all cascades showed subcluster formation above $200 \mathrm{keV}$ (Me76). The cluster size in gold increased with increasing primary energy until saturation, when splitting into subcascades occurred. Experiments on copper have not produced such definite results. It has not been determined whether the visible cascade probability goes to one with increasing bombardment energy or that the subcascade structure appears. In copper, no significant change in size distribution occurred with increasing self-ion irradiation energy, indicating possible subcascade structure developed at low recoil energies (Me74). It is believed that the defect density is too low for all depleted zones to collapse or, if collapsed, to be visible in the microscope. Some caution must be used in applying these results to predict neutron irradiation structure since the damage was produced close to the surface rather than in the bulk of the sample and surface effects may have perturbed the structure. Thus in summary, self-ion irradiations indicate that vacancy loops are the main defect cluster and that separation into subcascades 
occurred definitely in gold and possibly in copper.

Neutron irradiations produce a spectrum of recoll energies (see Figures 1 and 2), so the damage is more complex. Gold has been less comprehensively studied than copper, but several neutron Irradiation experiments have been conducted (Me74, Ea69, No69, Me65). Since self-ion irradiations determined the visible defect probability versus recoll energy in gold, knowledge of the neutron spectrum, and therefore the recoil energy spectrum, allowed comparison of the number of vistble defects with the number of theoretically predicted defects in gold. As in self-ion irradiations, the only clustered defect was reported to be of vacancy type. Merkle (Me74) also zeported that the density of clusters was a linear function of the $14 \mathrm{MeV}$ dose over the range $7 \times 10^{15} \mathrm{~cm}^{-2}$ to $2 \times 10^{16} \mathrm{~cm}^{-2}$. The cross sections for the fortation of a visible cascade frot experiments in a fission reactor (Ne65) and 14 MeT spectra (Me74) were in agreeaint with the theoretically predicted cross sections: experimental values of 0.55 and 3.3 barns compared favorably with the theoretical values of 0.60 and 3.0 barns for fission and I- $\because E Y$ speczze, respectively.

The first study o: neutron danage using Ten in copper was carrier out by Silcox and Hirsch (Si59). The size and density of Eisiocation loops were found to increase with dose until saturation at a fluence of $1.4 \times 10^{18} \mathrm{~cm}^{-2}$. rakin et al. (Na61, Ma62, 
Ma63) in a series of 1nvest1gations found that the defect size and density increased with 1ncreasing dose with no saturation for fluences between $6 \times 10^{17}$ and $4 \times 10^{18} \mathrm{~cm}^{-2}$. Small defect clusters ( $\dot{d} \leq 75 \dot{A}$ ) were found to increase linearly with dose, while largcr defects $(d \geq 75 \AA$ A) increased in density slightly less than linearly. In addition, Makin et al. found that the vistble defect size distribution was described by an expotential function:

$$
N(d)=N_{0} \exp \left(-d / d_{0}\right)
$$

where $N(d)$ is the number of defects of diameter $d$ and $N_{0}$ and $d_{0}$ are constants. This observation was later confirmed by Rühle et al. (Ru70), who found that the exact value of these constants depends on the irradiation conditions, particularly sample purity and temperature.

The observed nature of the defect clusters in copper has not been so consistent. Silcox and Hirsch (Si59) first identified the defects as dislocation loops. Makin and Manthorpe (Ma63) determined by annealing experiments that small clusters ( $d \leq 50 \AA$ ) were all of the same type (vacancy). The larger clusters $(\mathrm{C} \geq 50 \mathrm{~A}$ ) were of the opposite type, therefore interstitial. With the development of black-white contrast theory and the stereotechnique, a controversy arose as to the true nature of the defects: McIntyre (Mc67) reported the observation of only interstitial clusters, while Rühle 
and Wflkens (Ru67) reported defects only of vacancy character. Ipohorsk1 and Brown (Ip70) observed both types; of defect clusters with vacancy loops ( $\leq \leq 60 \AA$ ) comprising only $8 \%$ of the total population. In addition, wh1le Bourret and Dautreppe (B067) found that gaseous impurities were the cause of different concentrations of vacancy and interstitial loops, Ipohorski and Brown found that the mechanism for loop formation was not very sensltive to 1mpurities. Rühle and coworkers (Ru70, Ru70a) resolved the controversy by dolng careful measurements on samples irradiated at different factlities. The loops were divided into interstitial and vacancy, with vacancy loops dominating the small end $(d \leq 75 \AA$ ) of the distribution. Thus, McIntyre's data with loop diameters greater than $60 \AA$ and Rühle and Wilkens' (Ru67)

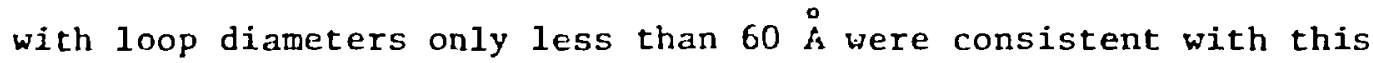
interpretation. In addition, Rühle et al. (Ru70) pointed out that dfference in the relative ratios of vacancy and interstitial clusters from one group to the other is dependent on the neutron spectrum, the irradiation temperature, and the sample purity.

One more recent application of TEM to damage structure was the comparison of numbers of retained point defects due to fission and $\operatorname{Be}(d, n)$ neutron sources. Roberto, Narayan, and Saltmarsh (Ro75a) found there were general similarities in the loop size distributions for the high energy $B e(d, n)$ and fission 
neutrons, but the number of retained defects was three times h1gher for Be(d,n) neutrons. The calculations of Fu and Ferey (Fu76) and Roberto and Robinson (Ro76) may be used to otlain the ratio of displacement cross section for $\operatorname{Be}(d, n)$ and fission neutrons ( 3.4 ) which compares favorably with the measured rat 10 of retained point defects (3.7).

$\mathrm{X}$-ray studies of defect clusters have been carried out on neutron-1rrad1ated copper (Gr70, Ba68, La71, Gr74, Th71, La75, La77, De73). These results for defect size, concentration, and type are in general agreement with TEM results. An approximately linear increase in defect density with dose has been observed (Ba68, Th71, La71, La77). Additiona11y, it has been confirmed that the dominant defects are dislocation loops with the larger defects interstitial in nature. Also it has been determined that the loops lie in [111] planes (De73, Gr74). The total number of defects stored in loops as measured by $X$-ray techniques is Iarger than that observed in IEx work. This is presumably due to the detection of small clusters $(d<20 \AA)$ by $x$-rays which were not distinguishable in TE measurements. Roberto et al. (Ro75a) Eeasured the retained deject concentratica in Ee(c,a) and fissiou neveros izraciated copper by X-ray diffuse scaterine. The ratio of retained damage Be(c,n)/fission was founc to be 3.3 , in good agreement hith the caiculated displacement cross section ratio of 3.4 
Thus the general consensus of these Irradiation experiments is that vacancy clusters are the dominant defect at these dose levels ( $10^{17} \mathrm{n}^{-2}$ ), but interstitial clusters may form under favorable condtions. The form of the defect clusters is dislocation loops on $\{111\}$ planes. Additionally, the ratio of retalned defects in copper irradiated with fission and $B e(d, n)$ neutrons is in good agreement with NRT model calculations.

\section{Electrical Resistivity Experiments}

Induced electrical resistivity is frequently used to deteraine the defect concentration during irradiation. The advantages of this parameter are the ability to make measurements during neutron irradiation and to make multiple measurements on the same sample. Furthermore, there is a simple relation between the irradiation induced resistivity and the defect concentration. The experimental damage rate can be expressed as:

$$
\rho=\rho_{F} \sigma_{d} \phi
$$

where $P_{F}$ is the Frenkel pair resistivity, $\sigma_{d}$ is the displacement cross section, and $\phi$ is the flux. The defect concentration, $c_{i}$, created by the irradiation is the rotal induced resistivity, $\rho_{i}$, divided by the Frenkel pair resistivity: $c_{i}=\rho_{i} / \rho_{F}$. One assumption maje in resistivity studies is that any defect clustering is small enough so that the cluster resistivity may be approximated by the single defect resistivity times the number of defects 
In clusters.

Burger et al. (Bu64) observed a linear decrease in the rate of change of the 1nduced resistivity with increasing fluence, except for an Initial transient, for reactor irradiated copper, gold, and several other materials at $4.2 \mathrm{~K}$. Horak and Blewitt (Ho72) studied Argonne CP-5 reactor irradiated metals and found the functional dependence suggested by Burger to be incorrect. They suggested a parabolic dependence of the damage rate on the induced resistivity, and found that several metals $(\mathrm{Al}, \mathrm{Cu}, \mathrm{Ag}$, Fe) fit this supposition. For other materials, either the data did not fit a parabolic equation ( $\mathrm{Ni}, \mathrm{Mo}, \mathrm{Co}$ ) or the scatter in the data was too large to determine if a valid fit existed ( $\mathrm{Au}$, Pt). Additionally, Horak and Blewitt observed that the saturation resistivity values were in general larger in Burger's irradiations than in their irradiations. The explanation for this difference lies in the spectrum of neutrons used. The reactor utilized by Burger had almost equal fluxes of thermal and fast ( $\mathrm{E}>0.1 \mathrm{MeV}$ ) neutrons, while the CP-5 facility had a flux of $1.3 \times 10^{12} \mathrm{~cm}^{-2} \mathrm{~s}^{-1}$ ( $\mathrm{E}>0.1 \mathrm{MeV}$ ) versus a thermal f.lux of $10^{8} \mathrm{~cm}^{-2} \mathrm{~s}^{-1}$. The number of isolated pairs and small defect clusters was larger in Burger's samples than in Horak and Blewitt's samples. The lower saturation resistivity in the samples of Horak and Blewitt was due to the breakdown of linearity of cluster size and cluster resistivity assumed in 
using equation I-13.

These studies polnted out the problem of separation of effects due to thermal and fast neutrons. Thermal neutrons produce low energy recolls from the $(n, y)$ reaction, thus producing only a few isolated Frenkel pairs. Fast neutrons create high energy cascades with the production of depleted zones and isolated defects. Horak and Blewitt (Ho73, Ho75) studied the difference in resistivity change and annealing properties of materials irradiated to the same fluences of fast and thermal neutrons. Only the results on copper and gold will be discussed here.

A distinction between the interstitial concentration observed in electrical resistivity measurements and that observed in our experiment will be discussed at this point. Irradiation induced interstitials surviving athermal recombination are available for annihilation in stage $I$. Some interstitials annihilate with their associated vacancy through close pair recombination and have practically no chance of escape. Other interstitials are sufficiently distant from their associated vacancy that recombination is likely but escape has a higher probability than in close pair recombination. This is referred to as correlated recovery and occurs in substage $I_{D}$. A third method for annihilation is uncorrelated recovery of an interstitial with a vacancy other than its associated vacancy occurring in 
substage $I_{E}$. The subset of interstitials that survive all of stage I annealing and randomly migrate in the lattice are the free interstitials measured in our experiment. To distinguish this subset from the total number of interstitials surviving athermal recombination, the adjective free will be used for the subset and the adjective "free" with the quotes w111 be used in the other case. The expression "free" is used to denote the high probability of recombination of interstitials in stage $I$. In copper, the stage I annealing for thermal neutron irradiation gave a $58 \%$ recovery with a peak at $48 \mathrm{~K}$. For fast neutron irradiation, this stage gave a $31 \%$ recovery with a peak centered at $38 \mathrm{~K}$. Stage I annealing is dependent on the number of "free" interstitials produced by the radiation. The smaller recovery and lower temperature peak of stage I in the fast neutron case indicates that fewer "free" interstitials were created than in the thermal neutron irradiation. In stage II (65 to $170 \mathrm{k}$ ) no recovery was observed for thermal neutron damage. A $30 \%$ recovery was observed for fast neutron irradiation, indicating that this stage was dependent on defect clusters absorbing or emitting interstitials. In stage III (170 to $320 \mathrm{~K}$ ) annealing, the thermal neutron peaks were at slightly higher temperatures than the fast neutron peaks with both irradiations giving $\sim 30 \%$ recovery. The defect concentration dependence of these temperatures showed again fewer 
avallable defects in the fast neutron case. Stages IV(300 to 450K) and $V($ above $450 \mathrm{~K}$ ) were both absent in thermal neutron recovery, but stage $V$ annealing did occur for fast neutron irradiation. Complete recovery of the resistivity for thermal neutrons was accomplished at $400 \mathrm{~K}$, but after annealing to $553 \mathrm{~K}$, $9 \%$ resfdual resistivity was unreccvered in fast neutron irradiated copper. The residual resistivity was not entirely due to transmutations, but probably due instead to small clusters that remain after annealing. Since stage I is due to "free" defects, we see a rough 2 to 1 ratio in the production of "free" interstitials by thermal and fast neutrons in copper. This was in agreement with the findings of Coltman et al. (Co62) that a fission neutron is $54 \%$ as efficient as an $(n, \gamma)$ event in producing resistivity change.

Gold has very different recovery stages due to thermal and fast neutrons. Stage I recovery in gold was evident for reactor irradiations with all annealing peaks occurring below $50 \mathrm{~K}(\mathrm{Sc} 69)$. These substages were confirmed using thermal neutron irradiation (C067). Horak and Blewitt (Ho73) showed that for fast neutron irradiation of gold, no distinct recovery processes were observed below $50 \mathrm{~K}$. Thus the recovery peaks in stage I were associated with the defects produced by the $(n, \gamma)$ reaction, most likely "free" interstitials or possibly small clusters. The absence of this stage in fast neutron irradiated 
goid Indicated that the "free" Interstitial concentration was below the detectability 11mit of electrical resistivity measurements $\left(-10^{-9}\right.$ af of Frenkel pairs). Stages II and III in reactor (Sc69, Ta69) and fast (kio73) neutron irradiation were comparable with shifts in the peak temperature due to defect concentration differences.

The difference between gold and copper response to fast neutron 1rradiation was the difference in cascade structure. Sigmund et al. (S167) concluded that for irradiations of this energy, the defect spacing increased in materials of smaller $z$ (higher PKA initial recoll energy). In aluminium, Sigmund suggested that essentially no self-annealing occurred, while in the heavier materials such as gold, the local defect concentrations were so high that substantial annihilation of defects occurred. Sigrand estimated the defect concentration in aluminum to be $10^{-3}$ times saturation concentration $(.005)$ wile in gold the concentration was ten times the saturation concentration. Copper was an internediate case, i.e., saturated and unsaturated cascades were both present. Since stage I annealing was eztibuted if ins -igration of defects to sinks, the more hotogeneous tins defect concentratios, the larger the stage I recover: Thus in copper, we sat a decrease from $58^{\circ}$ to $31_{n}^{\text {r. }}$ recovery in going irow thermal to fast neutron irradiation anci in gold a complete absence of stage I recovery due a smaller or absent 
"free" defect concentration during fast neutron frradiation. Additional Information is obtainable from electrical resistivity measurements. In equation I-6 of the NRT model, $K$ was defined to be the displacement efficiency and equal to 0.8 . An experimental displacement efflciency can be defined by:

$$
K_{\exp }=(d \rho / d \phi) 2 E_{d} / \sigma_{D E} \rho_{F} \cdot
$$

For thermal neutron irradiations, the experimental efficiency approached $80 \%$ (Ho75), but for fast neutron irradiations, the range of $K_{\text {exp }}$ was 20 to $50 \%$. The damage efficiencies measured at $4.2 \mathrm{~K}$ (below stage $\mathrm{I}$ in many metals) indicate that the theoretical efficiency for fast neutrons is roughly twice the experimental value.

Comparisons of the relative damage rates of thermal, fission, "14 MeV", and d-Be neutrons have also been made. Robinson (Ro75) has compiled a table of several elements which have been irradiated by both thermal and fast neutrons (fission neutrons). The initial slope of the resistivity change versus fluence is called the "damage resistivity" cross section $\rho_{\mathrm{F}} \sigma_{\mathrm{d}}$ (see equation I-13). For copper and gold, the experimental ratios of the damage resistivity cross sections for thermal and fast neutrons were 37 and 9.2 , while the theoretical ratios were 76 and 9.6. The good agreement between experiment and theory for gold was believed to be fortuitous. In general, the theoretical ratio was two times the experimental ratio. Thus good agreement 
with the NRT model was not found for the thermal to fission ratios. Roberto et al. (Ro77) have studied the rat1o of damage rate in fission and d-Be spectra and found good agreement with the NRT modeI. For copper, niobium, and plat Inum, the damage resistivity was found to be linear with dose to $4 \times 10^{15}$ neutrons $\mathrm{cm}^{-2}$. The ratio of the specific damage resistivity for copper was $3.3 \pm 0.7$ and the calculated ratio was $2.9 \pm 0.4$. This agretment was also reflected in the ratios for platinum and niobium. A comparison of "14 MeV" neutron damage at RTNS-I and danage from $d-B e$ neutrons on dilute alloys of niobium, vanadium, and molybdenum with zirconium was done by Guinan and Violet (Gu77). The damage rates were essentially constant to a total fluence of $10^{16} \mathrm{~cm}^{-2}$ for the three materials. The damage ratio of $\mathrm{d}-\mathrm{Be} / " 14 \mathrm{MeV}^{\prime \prime}$ neutrons was in good agreement with the theoretical ratios for all three materials.

From electrical resistivity measurements of neutron damage, the following conclusions can be drawn: (1) The stage I annealing indicates that less "free" interstitials are produced by a given dose of fast neutrons than thermal neutrons. (2) The damage effectiveness of high energy neutrons is less than the $80 \%$ predicted by the NRT model. (3) The NRT model prediction for the ratio of fission or " $14 \mathrm{MeV}$ " neutron damage rate to d-Be neutron damage rate is in good agreement with experimental calculations. 


\section{Internal Friction Theory}

Polnt defects produced by Irradiation can be studied by monttoring the changes in internal friction and Young's modulus. A convenient measure of internal friction (damping) is the logarithmic decrement, $\delta$, defined as one-half the fractional change in vibrational energy per cycle:

$$
\delta=\frac{\Delta W}{2 W}
$$

where $W$ is the total energy stored in the sample when the strain is a maximum. The changes in Young's modulus and the decrement are caused by the interaction of irradiation produced point defects and dislocations.

The motion of a dislocation in a solid was first modeled by Koehler (Ko52) and refined by Granato and Lücke (Gr56) as a vibrating string in a viscous medium. By use of effective mass and an effective line tension, a mathematically simple model of dislocation resonance was developed. The equation of motion is that of a damped harmonic oscillator:

$$
A \frac{\partial^{2} y}{\partial t^{2}}+B \frac{\partial y}{\partial t}-\frac{\partial^{2} y}{\partial x^{2}}=\sigma_{o} b e^{i \omega t}
$$

where: $\quad A$ is the effective mass per unit length $\left(\pi \rho b^{2}\right)$

$\rho$ is the mass density

$b$ is the magnitude of the Burger's vector

$B$ is the viscous damping constant 
C Is the effective ine tension $\left[2 G b^{2} / \pi(1-\nu)\right]$

$\sigma_{0}$ is the amplitude of the harmonic stress

$w$ is the frequency of the applied stress

$y$ is the displacement of an elemental portion of the dislocat1uni

$x$ is the distance frow a d1slocation pinning point

$t$ is the time.

The main results of this theory (KGL theory) as applied to irradiation experiments are the dependence of the damping $\delta$ and Young's modulus $Y$ on the length 1 of dislocation 1 ines that are free to move. In the usual interprecation of KGL theory appropriate at low Erequency and low strain amplitude, the damping and modulus defect $\Delta Y / Y$ have the following dependencies:

$$
\begin{aligned}
& \delta \sim \omega \Lambda B 1^{4} \\
& \Delta Y / Y \sim \Lambda^{2} \\
& 1=.1_{0} /(1+n)
\end{aligned}
$$

where 1 is the average length of dislocation line between pinniag points, $\mathrm{n}$ is the number of pinning points added to the dislocation lines of initial average length $1_{0}, \Lambda$ is the areal dislocation density, and $\Delta Y=Y_{e}-Y$ where $Y_{e}$ is the elastic (fully pinned) modulus. The above equations predict that during irradiation, the decrement and modulus defect monotonically decrease as point aefects are added to the dislocation lines. 
Additionally, these equations 1mply that the decrement decreases as the square of the modulus defect.

Experiments by Simpson et a1. (S172, S172a, S173) showed in the low frequency range ( $\omega S 1 \mathrm{kHz}$ ) that the above predictions do not always hold. At the onset of Irradiation, the decrement Increased to a maxlmum and then decreased for extended irradiations. During this time the modulus defect was monotonically decreasing. Simpson and Sosin (S172) explained this discrepancy by introducing a modification to KGL theory incorporating defect dragging during dislocation motion. Now the viscous damping term can be expressed as the sum of the inttial viscous drag prior to irradiation $\mathrm{B}_{\mathrm{o}}$ and the drag due to addition of $m$ defects added during irradiation, $\mathrm{mB}_{\mathrm{d}}$. In this theory, the decrement and modulus defect are given by:

$$
\begin{aligned}
\delta & =\frac{\pi \mathrm{Yb}^{2} \Lambda^{2}}{2 \mathrm{C}^{2}}\left[1-\frac{1}{\mu}\left(\frac{\sin \mu+\sinh \mu}{\cos \mu+\cosh \mu}\right)\right] \\
\frac{\hat{A} Y}{\mathrm{Y}} & =\frac{\mathrm{Yb}^{2} \Lambda^{2}}{2 \mathrm{C} \mu^{3}}\left(\frac{\sinh \mu-\sin \mu}{\cosh \mu+\cos \mu}\right)
\end{aligned}
$$

where $\mu^{2}=\omega B I^{2} / 2 C$. Now the effect of adding "pinners" is to increase $f$ through the viscous drag term $E$, as the disiocation drags the defects along, thus keeping the same line length 1 . In Figure 5, a graphical representation of the decrement and modulus defect are given as a function of the parameter $\mu$. If the initial value of $\mu$ before irradiation $\left(\mu_{0}\right)$ is less than the value of 


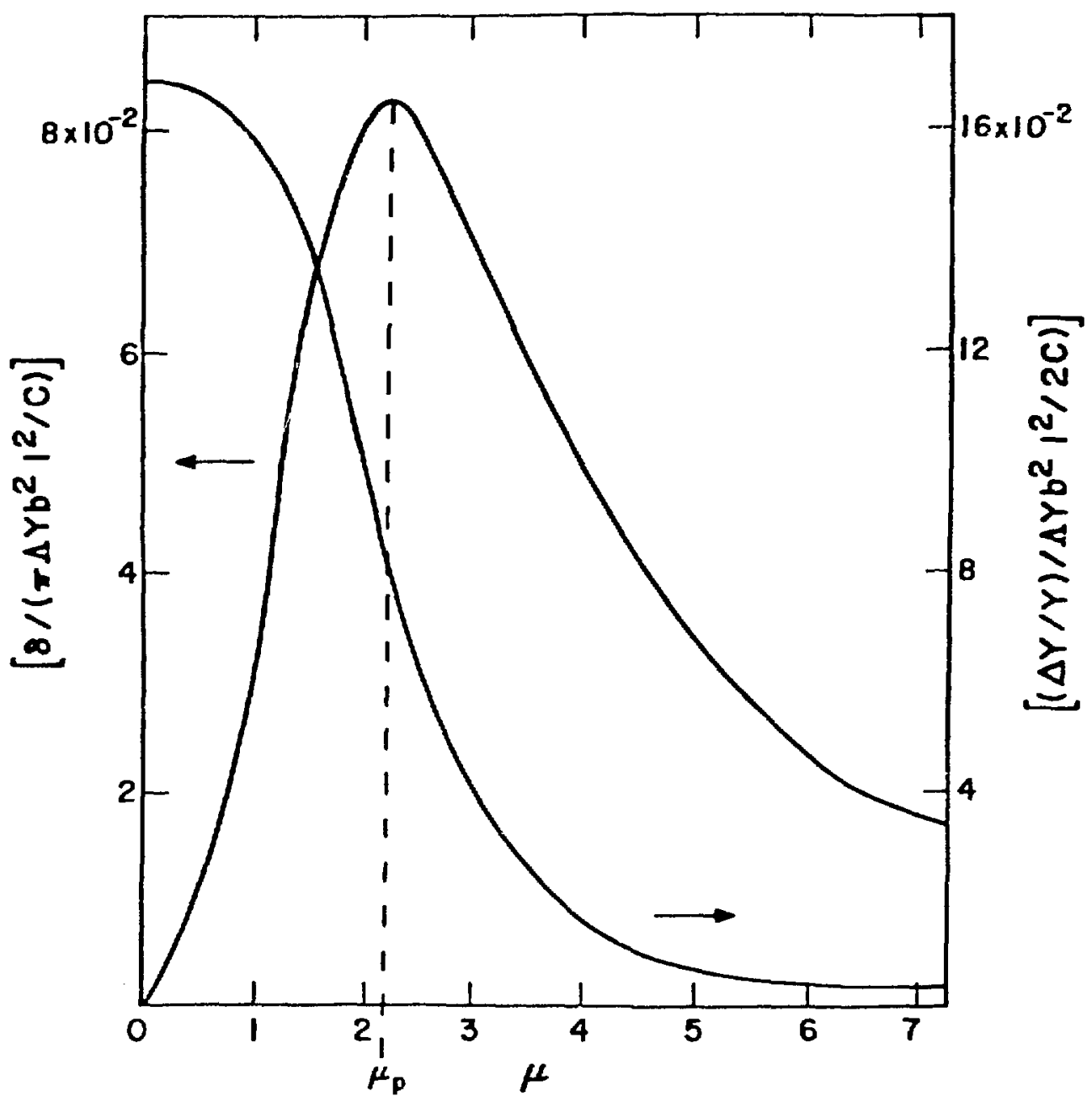

Figure 5: The decrement and modulus defect plotted as a function of the parameter $\mu$ of the Simpson-Sosin defect dragging model. 
$\mu$ at $\hat{o \partial} / \partial \mu=0\left(\mu_{p}\right)$, then the decrewent increases unt1l. the value $\mu_{p}$ is reached and then decreases. A large decrease in the modulus accompanies this decrement variation. If $\mu_{0} \geq \mu_{p}$, then both the modulus defect and the decrement monotonically decrease.

\section{E. Internal Friction Measurements}

Internal friction and Young's modulus experiments offer the most sensitive technique for the study of radiation damage. Dislocations contribute an Inelastic strair. under apjifed stress causing the measured elastic modulus to be smaller than the true value. Since the resonant frequency of a sample is dependent on the elastic modulus, a change in the strain contribution by dislocations will be reflected in a change of the resonant frequency. The decrement is associated with the energy loss and is a measure of phase difference between the applied stress and the strain. The decrement is measured through the change in the drive voltage necessary to maintain constant strain. The suppression of dislocation motion, therefore, will sause an inscease in Young's modulus and a change in the dectement.

The aim of nost internal friction experiments was not to study defect production rates but to compare results with dislocation wodels. An excellent review by Sosin and keefer (5o68) 
has been published. In general, above frequencles of $20 \mathrm{kHz}$, the damping and modulus defect were found to be proportional to dislocation line length in agreement with the string model. But varlous attempts to establish the low frequency dependence of dislocation motion in the hertz and low kllohertz range found departures from the vibrating string model. The modulus defect showed little frequency dependence, but the damping leveled off or increased at low frequenctes.

The most comprehensive study of the internal friction behavior at roughly $1 \mathrm{kHz}$ has been conducted by Dr. H. M. Simpson and co-workers on electron irradiation of a cantilevered beam sample. Electron irradiations of copper samples have shown several characteristics of the peaking effect of the decrement. Since the first proposal of the defect dragging mechanism (Si71), it was evident that the effect was dependent on the pre-irradiation annealing history of the sample. For high temperature anneals prior to irradiation, the peaking effect was absent during irradiation presumably due to an increase in $\mu$ through an increase in the dislocation line length. Later experiments (Si72a) showed the critical annealing temperature for eliminating the peaking effect in copper was about $1023 \mathrm{~K}$. Studies of the temperature dependence of the nodulus defect (Si72a, Si73) yielded information on the migration of the dragging defect. The Arrehenius plot of inverse modulus defect $\left.\left.\left[(\Delta Y / Y)_{0} / \Delta Y / Y\right)-1\right)\right]$ or the steady 
state pinning point concentration gave a value for the difference between the lattice migration enthalpy and the migration enthalpy along dislocations of $0.17 \mathrm{eV}$ in the temperature range above $300 \mathrm{~K}$. An important study was conducted by Simpson and Kerkhoff (Si74, S176) on irradiation and quenching effects on the damping and modulus defect. By studying samples that had been electron irradiated, quenched and then electron irradiated, and only quenched, they were able to determine that the Interstitial defect was responsible for dragging above room temperature. The absence of the peaking effect in only quenched samples indicated that the vacancy-dislocation interaction sufficiently limits the drag so that the peaking effect did not occur. Since samples after quenching showed a reduced, but significant, peaking effect during electron irradiation, it was evident that the interstitial must be the dragged defect. The determination that the peaking effect is an indicator of interstitial "pinning" is, for the purpose of the present experiment, the most important conclusion of the electron irradiation experiments.

Neutron irradiation studies of dislocation damping have Eainly concentrated on comparison to $K G_{L}$ theory. All experiments have been conducted in a reactor environment. Thompson and Holmes (Th56) using vibration frequencies in the 10 to $50 \mathrm{kliz}$ range first measured the variation of both damping and modulus defect during reactor irradiation. They were able to interpret their results in 
terms of the reduction of the average loop length by creation of additional pinning points. Agreement with KGL theory was good except for the frequency dependence of the decrement. Experiments using megaiertz frequencies have shown complete agreement with KGL theory (Al61, He67). An extensive study of dislocation pinning in copper by neutron irradiation has been done by Paré and coworkers (Th60, Pa62, Pa73, Pa74a) utilizing Oak Ridge Irradiat Ion factlities. The two main points of study were the temperature dependence of the pinning rate and the comparison with KGL theory. The temperature depentence data from 20 to $410 \mathrm{X}$ disagreed with similar data from electron or gamma irradiations. In particular, no decrease in the pinning rate was observed in the neighborhood of $160 \mathrm{~K}$, thus indicating that defect production may be different for neutron irradiations. To explain the discrepancies with the KGL model, Faré and Thompson (Pa62, Th60) assumed a two-component dislocation model. This was necessary to explain the difference in the number of pinning points derived from the decrement and modulus defect data. In an attempt to reconcile the $\mathrm{kHz}$-frequency data with KGL theory, Paré and Guberman (Pa73) examined the various alternates. lio proposed modification of the KGL model gave completely satisfactory results. In particular, if kú theory was used, the dislocation viscous damping coefficient had to assume an unteasonably high value in comparison with measurements from the Hz frequency range. If the damping coefficient 
was unaltered, the predicted loop length was unsatisfactory. The defect dragging model of Simpron and Sosin also had physically questionable values for either the damping due to defect drab or the amplitude of motion of the dragged defect. Paré never raported an ircrease In damping during irradiation, which might have been expected if the Simpson-Sosin model applied.

Thus the controversy over dislocation models and alternative modifications to models has not been resolved. The apflicabllity of KGL or Simpson-Sosin models to irradiation experiments using internal friction techniques appears to be limited. All experiments have shown that the modulus defect decreases monotonically indicating that the dislocations are constrained by discrete pinning points. If dislocation mechanics are assumed valid, the area swept by dislocations between pinning points must be predicted in some manner by the KGL model. The pinning points must be fairly effective since the dislocation motion can be fully restrained by fairly low pinnjing point densities. Thus, whatever the problems with KGL or Simpson-Sosin models, the modulus defect data can be used relatively confidently as a measure of pinning point density even though the decrement data would lead to serious inconsistencies.

F. Present Experiment

The main objective of this experiment was twofold: (1) to 
study the neutron energy dependence of the number of point defects that pin dislocations and (2) to study the correlation between the predicted and measured number of defects. Point defects produced by frradiation were studied by monftoring the changes in internal friction and Young's modulus of a foll specimen that was mechanically vibrating. These changes can be related to tine number of pinning points added to a dislocation line. The damage was annealed out in situ so as not to disturb the dislocation structure. In addition to neutron irradiations, electron bombardments of the same sample were conducted. Since the neutron flux levels were too low to obtain, within a reasonable length of time, eiastic (fully pinned) values of Young's modulus and the decrement necessary for the calculation of the pinning rate, electron irradiations provided this information. Also, electron irradiations were a calibration of the pinning rate during irradiation versus the number of free interstitials produced.

This technique offers several advantages over previous datage studies:

(I) The sensitivity of Young's modulus to low defect concentration. ( 1 part in $10^{13}$ ) ensures that complications due to cEEes: irterctions do not arise. This is consastec hitt. ejectricai resistivity measurements comoniy done on neviror irzadicted samples wiere concentrations of 1 pert in $10^{E}$ are necessary. Due to the lor flux: levels ( $10^{8}$ neutrons $\mathrm{cm}^{-2} \mathrm{~s}^{-1}$ ) of ponoenergetic 
neutron sources, a sensitive parameter is necessary for these measurements.

(2) The relation between Young's modulus and the number of defects on a dislocation line, $n_{d}$, is relatively simple (S172a):

$$
n_{d} \propto \frac{(\Delta Y / Y) 0}{(\Delta Y / Y)}-1
$$

where the subscript o Indicates the pre-irradiation value and $\Delta Y=Y_{e}-Y$ where the subscript e indicates the elastic (fully pianed) value.

(3) The use of monoenergetic neutron sources reduces the complexity of the experiment. One can pointwise compare theoretical damage cross sections and experimental data without having to integrate over a neutron spectrum. Furthermore, the measurement of the neutron flux by dosimetry foll techniques is simplified by use of monoenergetic neutrons.

(4) In comon with electrical resistivity measurements, continuous bonitoring of changes during neutron irradiation is possible. Furthermore, internal friction techniques have the advantage of continuous monitoring during electron irradiations.

(5) The same sample is used throughout the experiment For both electron and neutron irradiations. In order to perserve the cislocation structure and density, the sample is annealed between irradiations at $\sim .6 \mathrm{~T}_{\mathrm{m}}$ to eliminate only defects created by the irradiation. By measuring the flux and temperature 
dependence of the pinning rate by electron and neutron 1rradiations, supplemented by the observation of the peaking effect, one can determine that the same defect, the free interstitial, is responsible for pinning dיring both 1rradiations.

(6) Finally, a direct comparison of damage rates by electrons and neutrons can give estimates of the average number of free interstitials produced per cascade at a given neutron energy. 


\section{Experimental Procedures}

\section{A. Sample. Preparation}

Two materlals were studied in this experiment. The copper was $99.999 \%$ pure extruded rod purchased from Amertcan Smelting and Refining Company. The gold rod was $99.999 \%$ pure material purchased from Research Organic/Inorganic Chemical Corporation. Typical analyses of the above materials as furnished by the manufacturers are given in Table 2. The samples were machined in the form of cantilevered beams with a flexural resonant frequency around $1 \mathrm{kHz}$. After machining, the samples were hand-polished and electropolished to their final thicknesses. The base of a sample was $1.27 \mathrm{~cm}$ long, $0.64 \mathrm{~cm}$ wide, and $0.32 \mathrm{~cm}$ thick while the vibrating beam, continued from the base, was $0.64 \mathrm{~cm}$ square and $0.0076 \mathrm{~cm}$ thick. The relation between Young's modulus, $Y$, and the resonant frequency of the sample, $v_{r}$, is $(M 048):$

$$
\nu_{T}=\frac{0.55966}{1^{2}} \frac{t}{\sqrt{12}}\left(\frac{Y}{\rho_{m}}\right)^{1 / 2}
$$

where $\rho_{\mathrm{m}}$ is the mass density, 1 is the length of the sample, and $t$ is the thickness parallel to the direction of vibration. For $\mathrm{Y}=$ $4 \times 10^{11}$ dynes $/ \mathrm{cm}^{2}$ and $\rho_{\mathrm{m}}=8.9 \mathrm{gm} / \mathrm{cm}^{3}, \nu_{\mathrm{r}} \cong 650 \mathrm{~Hz}$ for the copper sample. Actual frequencies for both the gold and copper samples were around $770 \mathrm{~Hz}$.

Since the same sample was used in each set of experiments, 
TABLE 2

TYPICAL ANALYSES

\section{Copper $99.999 \%$}

$$
\begin{aligned}
& \mathrm{Mg} \text { very faint trace } \\
& \mathrm{Ag} 0.1 \mathrm{ppm} \\
& \mathrm{Se} \text { and } \mathrm{S} \quad 1 \mathrm{ppm}^{*}
\end{aligned}
$$

Not detected by spectrographic analysis:

$\mathrm{As}, \mathrm{Bi}, \mathrm{Cd}, \mathrm{In}, \mathrm{Mn}, \mathrm{N} 1, \mathrm{~Pb}, \mathrm{Sb}, \mathrm{Sn}, \mathrm{Te}, \mathrm{Tl}$

* Chemical analysis

\section{Gold $99.999 \%$}

A1 $0.2 \mathrm{ppm}$

Ca $0.4 \mathrm{ppm}$

$\mathrm{Cu} \quad 0.2 \mathrm{ppm}$

Fe $0.2 \mathrm{ppm}$

$\mathrm{Pb} \quad 0.3 \mathrm{ppm}$
$\mathrm{Mg} \quad 0.1 \mathrm{ppm}$

Pd $0.2 \mathrm{ppm}$

Si $0.2 \mathrm{ppm}$

$\mathrm{Ag} \quad 2.0 \mathrm{ppm}$ 
measuring the dislocation density by a destructive technique was Impossible. The sensitivity of $x$-ray techniques border on the estimated density $\left(\sim 10^{9} \mathrm{~cm}^{-2}\right)$ of dislocations in the sample. Since each sample was annealed at $773 \mathrm{~K}$ for 30 minutes prior to any Irradiations and this temperature was never exceeded, the dislocation structure was approximately the same for every irradiation. The analysis of the experimental data and the use of the same sample eliminates the need to know the exact dislocation parameters.

The sample and the drive plate, attached to but insulated from the sample by a thin sheet of mica, formed a capacttor and were mounted on the end of a copper cap (see Figure 6). The cap was pressed onto a thin-walled $9.5 \mathrm{~mm}$ stainless steel tube for support. Shielded nichrome heater wire was threaded onto the cap. A chromei-alumel thermocouple was attached to the cap for temperature measurement and control. A small diameter (3.2 mm) stainless steel tube ran coaxially with the larger tube, almost to the copper cap, for cooling purposes. This sample rig was mounted in a stainless steel vacuum chamber which was held at $10^{-6}$ torr or bettei by an ion pump. The vacuum system was used to eliminate air damping and prevent oxidation during annealing; and the fon pump was used to prevent extraneous vibrations. 

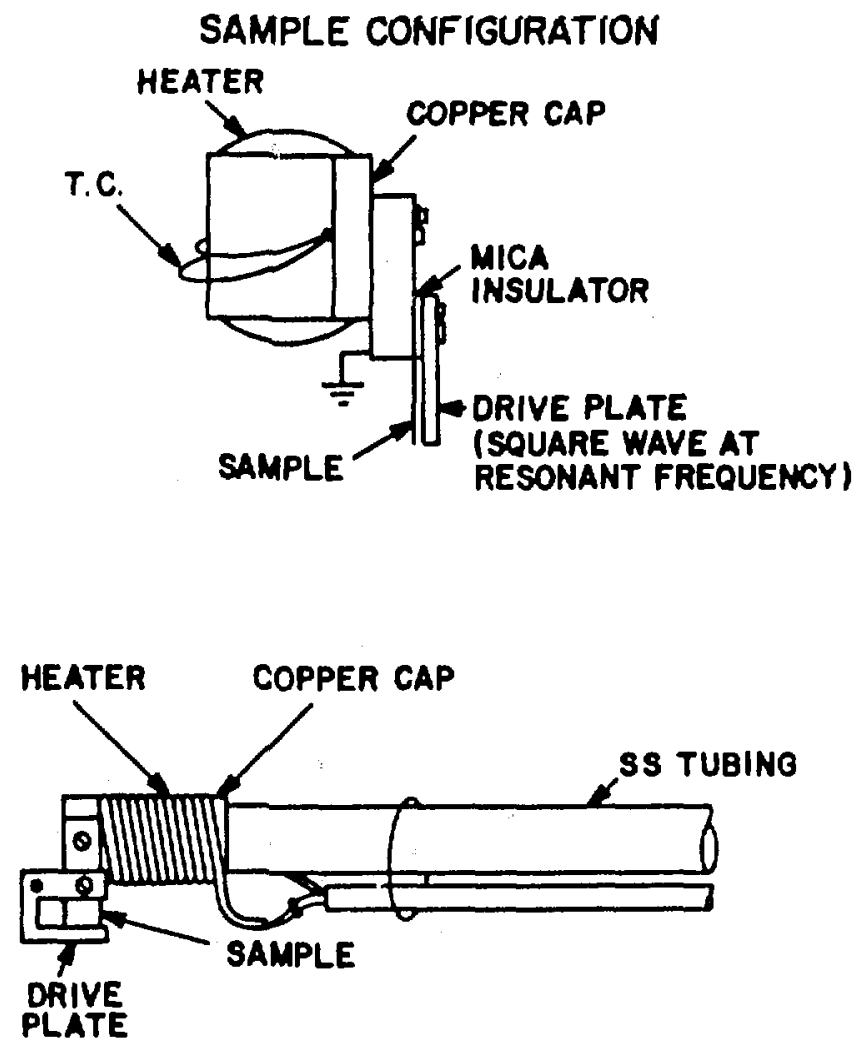

Figure 6: The sample and drive plate mounted to the copper cap at the end of a stainless steel tube. A nichrome heater was used for temperature control and annealing. 


\section{B. Electronics}

A square wave voltage of frequency $\nu_{r} / 2$ applied to the drive plate forces the sample to oscillate at frequency $\nu_{r}$. Since the sample acts as a high $Q$ filter, it sees mainly the Fourler component of the square wave at its resonant frequency. The sample is placed in the tank circuit of a FM oscillator (see Figure 7) via a half-wave coaxial cable. As the sample vibrates, the capacitance in the tank circuit changes and the FM signal is modulated. From Figure 7 , a change is $C_{s}$ due to a sample deflection $\delta d$ is:

$$
\delta C=C_{s} \frac{\delta d}{d}
$$

for a parallel plate capacitor. This change leads to a modulation of the oscillator frequency of:

$$
\delta \nu=\frac{1}{4 \pi \sqrt{L C}} \quad \frac{\delta C}{C}=\frac{\nu_{0}}{2} \frac{\delta C}{C}
$$

where $1 / C=1 / C_{1}+1 /\left(C_{2}+c_{s}\right)$. Since $c_{s} \ll c_{1}$ and $c_{1} \simeq 20 C_{2}$, then:

$$
c=c_{2}+c_{s}
$$

and

$$
\delta \mathrm{C}=\delta \mathrm{C}_{\mathrm{s}}
$$

so

$$
\delta v=\frac{v_{0}}{2} \frac{C_{s}}{C_{2}+C_{s}} \frac{\delta d}{d} .
$$

A MacIntosh MR77 FM tuner demodulates the FM signal. The output of the tuner is at the sample resonant frequency and the amplitude 


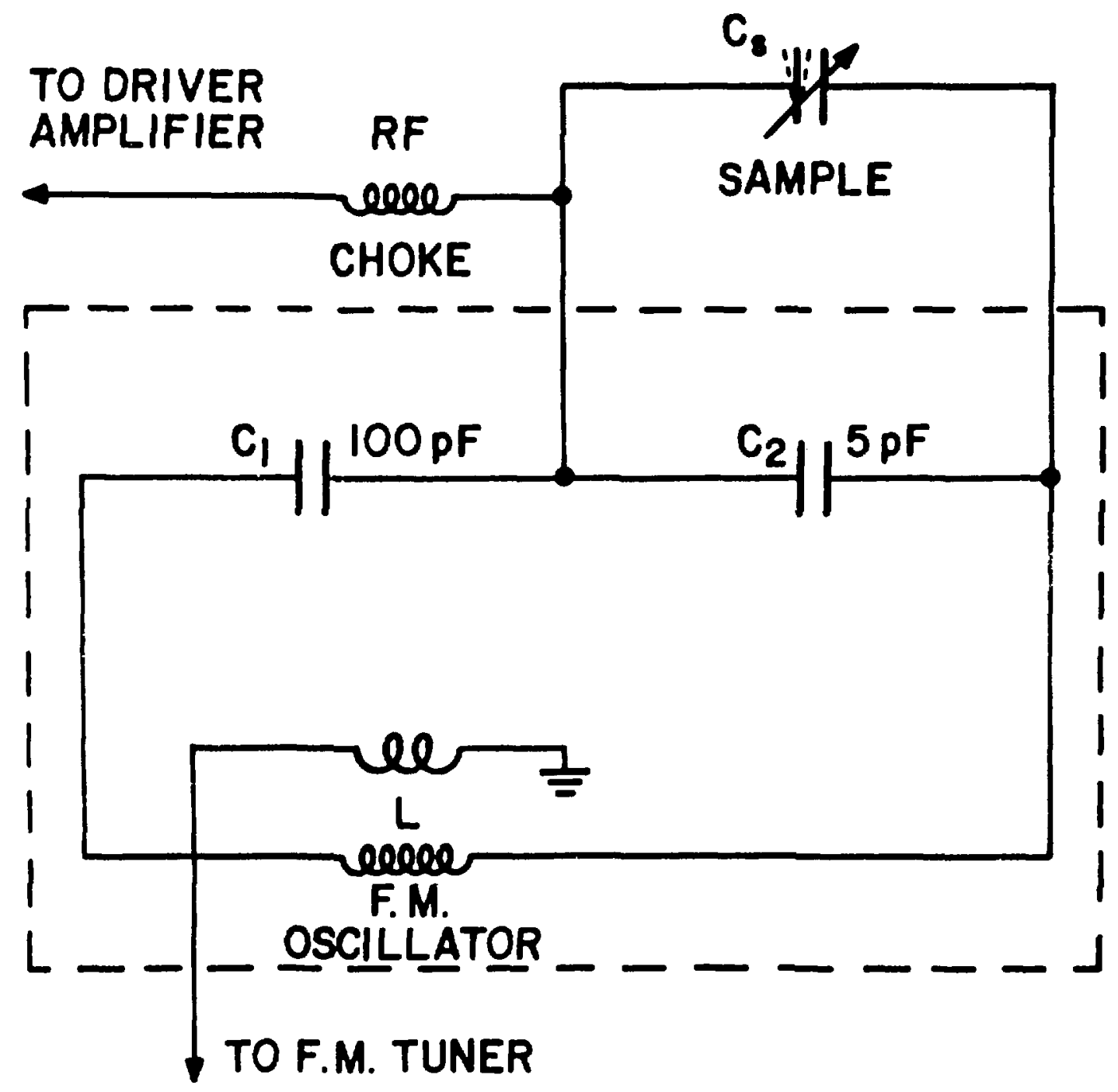

Figure 7: The sample is a variable capacitor in the tank circuit of a FM oscillator. As the plate separation changes the oscillator frequency is modulated. 
$\left(V_{t}\right)$ is proportional to the sample amplitude. The proportionality is dependent on the demodulation sensitivity $\left(S_{d}\right)$ of the FM tuner. For the MR7?, $S_{d}=2.5$ volts at $75 \mathrm{kHz}$ modulation. So:

$$
\delta v=v_{t} s_{d}
$$

Figure 8 shows the remaining electronics which monitor and record the period of vibration and the drive voltage (Si77). The period is measured rather than the frequency since by averaging over many $\left(10^{3}\right.$ to $\left.10^{4}\right)$ perfods better accuracy is obtained. The fixed output of the tuner goes to a bandpass filter (Kronhite 3103) to clean up the signal. The bandpass filter output goes into two components of the phase-locked-loop system. One section goes through an amplifier and then to a phase-locked-loop I.C. (LM565) which tracks any changes in the sample resonant frequency and modifies the driver amplifler frequency accordingly. The second filter output goes to an $A C / D C$ converter (HP 400E) which monitors the tuner output voltage. The HP $400 \mathrm{E}$ output is sent to an amplitude controller to adjust the magnitude of the driver output so that the sample vibrates at constant amplitude. The variable output of the tuner is fed into an active filter (Tektronix module AF 501) and the processed signal can be observed on an oscilloscope.

Three parameters can be measured with this system. The period is averaged by a Tektronix DC 505A universal counter/timer 


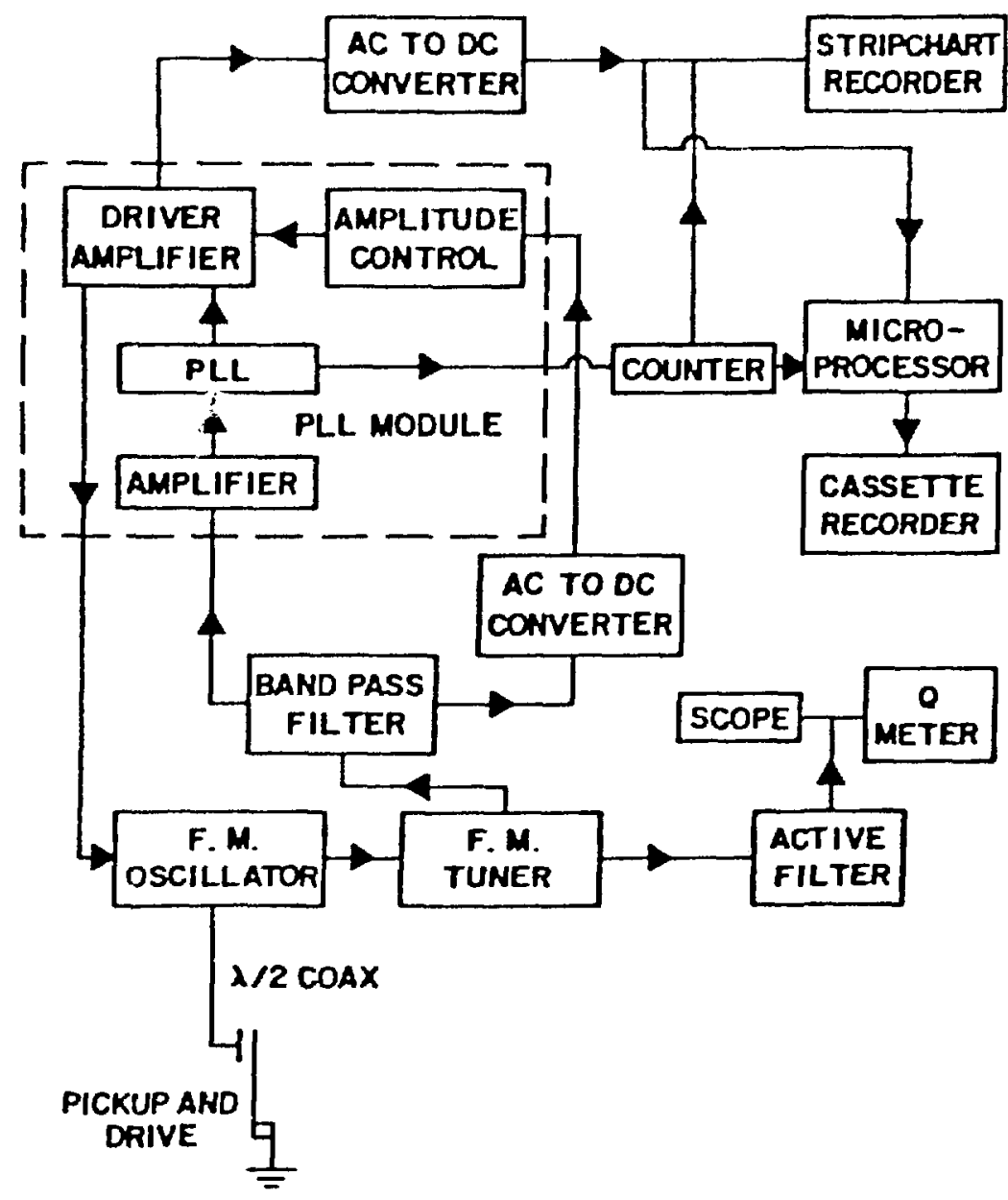

Figure 8: Flow chart of the electronics which automatically follow and record the changes in the decrement and Young's modulus. 
and an HP 5300 measurement system with a $5308 \mathrm{~A}$ universal counter/ timer and a 5311B D/A converter. Both modules average over at least $10^{3}$ periods with the DC 505A output sent to a microprocessor and HP 5300 output fed to a strip chart recorder. The drive voltage $1 E$ tracked by a Tektronix DM 501 digital voltmeter with an $A / D$ converter and an HP $400 E$ used as an AC/DC converter. The DM 501 output goes to the microprocessor and the $400 \mathrm{E}$ output goes to the strip chart recorder. The microprocessor samples the values of the DM 501 and DC 505A at intervals from 5 seconds to 5 minutes apart depending on experimental needs. The microprocessor writes the data on a cassette tape through a Texas Instruments 733 ASR terminal. The log decrement during free decay fan be measured from the digital display of the Q-meter or a photograph of the free decay from the oscilloscope.

The temperature of the sample is monitored by a chromelalumel thermocouple. The temperature controller detects the difference between the thermocouple reading and the set point on the LdN potentiometer (see Figure 9). The potentiometer output is detected with a null detector (Keithley 155) and an error signal is sent to the temperature controller. The controller attempts to null this error by increasing (or aecreasing) the current applied to the heater from an $\mathrm{HP} 6290 \mathrm{~A}$ DC power supply. The controller holds the set point temperature to less than $\pm 1 \mathrm{~K}$ over a range from room temperature to $773 \mathrm{R}$ (Si78). 


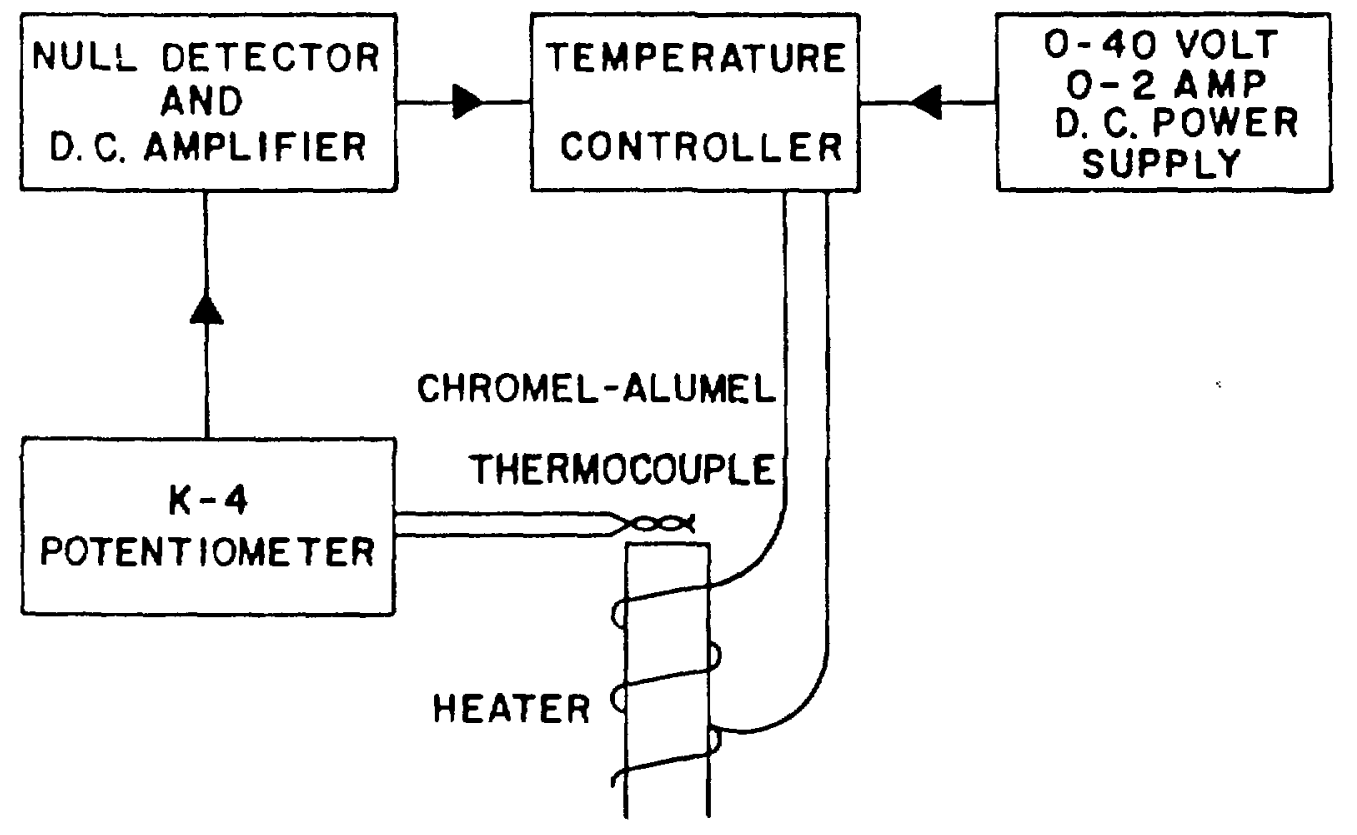

Figure 9: Flow chart of the temperature controller. 


\section{Calculation of Measured Parameters}

As the sample is driven in forced oscillation, it behaves as a damped harmonic osclllator. The equation of motion is:

$$
m \ddot{x}+B \dot{x}+m \omega_{0}^{2} x=F_{0} e^{i \omega t}
$$

where

$$
\begin{aligned}
B & =\text { damping constant } \\
m & =\text { the effective mass } \\
\omega_{0} & =\text { resonant frequency of the sample } \\
F_{0} e^{i \omega t} & =\text { driving force of anguiar frequency } \omega .
\end{aligned}
$$

Since the sample is driven at its resonant frequency, the maximum amplitude is:

$$
x_{0}=\frac{F_{0} / m}{\omega_{0}^{2} \delta_{0} / \pi}
$$

where $\delta=\pi B / \omega m$ is the decrement. Throughout the experiment, the amplitude of the sample is kept constant, so:

$$
\frac{F_{0} \pi}{\omega_{0}^{2} m \delta}=\frac{F \pi}{\omega^{2} m \delta}
$$

and

$$
\frac{\delta}{\delta_{0}}=\frac{\omega_{0}^{2}}{u^{2}} \frac{F}{F_{0}} .
$$

The sample is one plate of a parallel plate capacitor, therefore:

$$
F \propto v^{2}
$$


where $\mathrm{V}$ is the amplitude of the driving voltage. Thus:

$$
\frac{\delta}{\delta_{0}}=\frac{\omega_{0}^{2}}{\omega^{2}} \frac{v^{2}}{v_{0}}=\left(\frac{\tau v}{\tau_{0} v_{0}}\right)^{2}
$$

where $\tau$. is the period of oscillation $(1 / 2 \pi \omega)$. From equation II- 23 . we see that Young's modulus is proportional to the resonant frequency squared, so:

$$
\mathrm{Y} \propto 1 / \tau^{2}
$$

and

$$
\Delta Y / Y=\tau^{2} / \tau_{e}^{2}-1
$$

where $\Delta Y$ is equal to $Y_{e}-Y$ and $Y_{e}$ is the elastic value (fully pinned) of the modulus obtained from electron irradiations.

\section{Measurement of the Strain Amplitude}

The strain needs to be related to equation II-28 for a forced, damped harmonic oscillator. We can approximate the true situation by assuming that the force is applied only at the free end of the beam. The strain then depends on the displacement of the free end. Ignoring the effect of damping, we can approximate the vibrating sample by a bar with a static force applied. Then the strain is given by:

$$
\varepsilon=\frac{t}{2} \frac{d^{2} y}{d x^{2}}
$$

where $t$ is the thickness of the sample and $d^{2} y / d x^{2}$ is the 
approximation to the radius of curvature for small displacements:

$$
\frac{1}{R}=\frac{d^{2} y / d x^{2}}{\left[1+(d y / d x)^{2}\right]} \cong \frac{d^{2} y}{d x^{2}}
$$

where $(d y / d x)^{2} \ll 1$. The form of the fundamental mode is given by (No72):

$$
y=\frac{y_{0}}{2} \quad\left[\frac{\cosh (2 \alpha x / 1)}{\cosh \alpha}+\frac{\sin (2 \alpha x / 1)}{\sin \alpha}\right]
$$

where 1 is the length of the sample, $x$ is the distance along 1 measured from the middle of the sample, $y_{0}$ is the displacement at the free end of the sample, and $\alpha=0.29843 \pi$. Thus the strain at any point along the sample is given by:

$$
E=\frac{t}{2}\left(\frac{2 \alpha}{1}\right)^{2} \quad \frac{y_{0}}{2}\left[\frac{\cosh (2 \alpha x / 1)}{\cosh \alpha}-\frac{\sin (2 \alpha x / 1)}{\sin \alpha}\right]
$$

The maximum strain occurs at the point where the base of the sample begins, at $x=-1 / 2$ :

$$
\varepsilon_{\max }=1.76 t y_{o} / 1^{2}
$$

In equation II-26 the displacement of the sample given by $\delta d$ is shown to change the frequency of the FM oscillator. This relation can be measured. The sample is placed in the tank circuit of the oscillator and the end of the sample is displaced by means of a precision differential screw translator (resolution: $2.5 \times 10^{-6} \mathrm{~cm}$ ). As the end is displaced, the frequency is monitored on a $150-\mathrm{MHz}$ counter, so the proportionality between the sample displacement 
and the frequency modulation can be obtained. A $25 \mu \mathrm{m}$ displacement was found to change the frequency by $165 \mathrm{kHz}$ for the copper sample and $132 \mathrm{kHz}$ for the gold sample. For a tuner output of 0.05 volts and a demodulation sensitivity of 2.5 volts at $75 \mathrm{kHz}$, $\mathrm{y}_{\mathrm{o}}$ is:

$$
\begin{aligned}
y_{0} & =v_{t} s_{d} \delta \mathrm{d} / \delta \mathrm{v} \\
& =(0.05 \mathrm{~V})(75 \mathrm{kHz} / 2.5 \mathrm{~V})(25 \mu \mathrm{m} / 164 \mathrm{kHz}) \\
& =0.23 \mu \mathrm{m}
\end{aligned}
$$

and the maximum strain is:

$$
\begin{aligned}
& =1.76\left(2.3 \times 10^{-5} \mathrm{~cm}\right)(.0076 \mathrm{~cm}) /(.635 \mathrm{~cm})^{2} \\
& =7.5 \times 10^{-7}
\end{aligned}
$$

Similarly for gold, we find $\varepsilon_{\max }=5.0 \times 10^{-7}$. Thus the strain in the samples is on the order of $10^{-6}$. In Figure 10, the damping is shown as a function of the strain. $v_{t}$ is the tuner output voltage which is proportional to the strain and $V_{d}$ is the drive voltage which is proportional to $\mathrm{F}^{1 / 2}$. The operating point is shown at $v_{t}=0.05$ volts, and borders on the region of strong amplitude dependence. The strain is not quite in the amplitude independent region, but since the experiments are all done at the same strain this should not affect the results.

\section{E. Irradiations}

Electron irradiations were done at the $2.5 \mathrm{MeV}$ Van de 


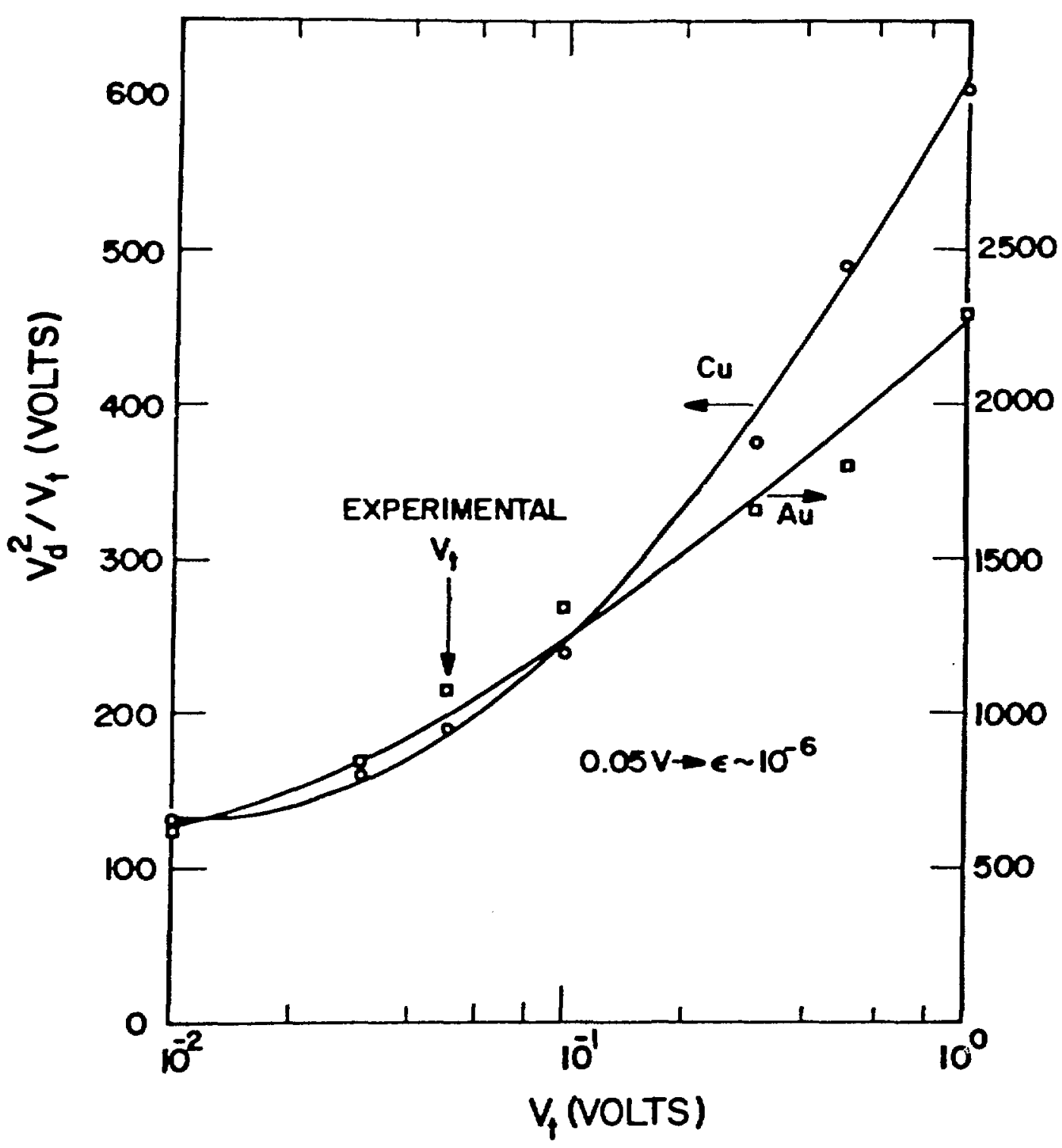

Figure 10: A plot of the driving force per unit strain versus the strain. $F \propto V_{d}^{2}$ and $\varepsilon \propto V_{t}$. The experimental value of $v_{t} 150.05$ volts which corresponds to a strain of roughly $10^{-6}$. 
Graaff at Wright State UnIversity under the supervision of Dr. H. M. Simpson of the Physics Department. For these Irradiations, the sample was mounted in the beam line in a vacuum of $10^{-6}$ torr. A square aperture divided into quadrants, which was mounted in front of the sample, monitored the uniformity of the beam and defined the beam size of $0.63 \mathrm{~cm}^{2}$. A Faraday cup was mounted behind the sample for current monttoring. The true beam current was found by grounding the entire sample chamber, including the drive plate and excluding the Faraday cup, and adding the current from the chamber to the Faraday cup current reading. The copper sample was irradiated with $0.5 \mathrm{MeV}$ electrons with intensities varying from $10^{9}$ to $10^{11} \mathrm{~cm}^{-2} \mathrm{~s}^{-1}$. Theoretical calculations of the range of $0.5 \mathrm{MeV}$ electrons in copper give values of $0.257 \mathrm{gm} / \mathrm{cm}^{2}$ (Be54). This translates to a thickness of $0.03 \mathrm{~cm}$. Experimental range values determined by Miller (Mi70) are 55 to $60 \%$ of the theoretical values or approximately $0.02 \mathrm{~cm}$ for $0.5 \mathrm{MeV}$ electrons. The difference between these values is due to Miller's extrapolation proct dure which actually gives a range value with $1.0 \%$ residual transmission. This number was actually more meaningful for this experiment since the homogeneity of the damage was important. For gold irradiated with $2.0 \mathrm{MeV}$ electrons, the theoretical range given by Berger (Bef4) is $1.56 \mathrm{gm} / \mathrm{cm}^{2}$ or $0.08 \mathrm{~cm}$ whereas the experimental result given by Miller is $0.563 \mathrm{gm} / \mathrm{cm}^{2}$ or 
$0.03 \mathrm{~cm}$. Since the sample thickness in both bases was $0.0076 \mathrm{~cm}$, the experimental range was larger and the damage was approximately homogeneous.

Neutron 1rradiations were done at three accelerators, two at Los Alamos Scientific Laboratory and one at the Mound Facility In Miamisburg, OH. The $14 \mathrm{MeV}$ neutron experiments were done at Pajarto Site, LASL, where $300 \mathrm{keV}$ deuterons produced by a Cockcroft-Walton accelerator bombard a tritiated solid target. By looking at $90^{\circ}$ to the beam direction, $14 \mathrm{MeV}$ nectrons were available to a flux of $2 \times 10^{8} \mathrm{~cm}^{-2} \mathrm{~s}^{-1}$ at the sample position, $3.2 \mathrm{~cm}$ away from the target center. Since the beam energy is very stable and small fluctuations do not change the neutron energy significantly, the major spread in energy came from the solid angle subtended by the sample. The fact that the sample sat tangentially to the equal flux surfaces produced some variation in the uniformity of the flux $(-2 \%)$. At this bombardment energy, the source was isotropic. Therefore, by using an associated alpha particle counter to measure the source strength, the flux at the sample was found by using the $1 / r^{2}$ law. The alpha particle counter had been calibrated by the Radiochemistry Group of LASL using Al dosimetry foils and found to be in agreement with the dosimetry results within $3 \%$. Since this facility offered ease of operation and on-line flux values, all flux and temperature dependence runs on copper were done there. 
Neutron energy dependence experiments with copper were conducted at the Tandem Van de Graaff at LASL w1th the ald of R. x. Smlth. Neutron energies from 2 to $24 \mathrm{MeV}$ were avallable by boubarding hydrogen and deuterium gas targets with tritons at appropriate energies. In F1gure 11, the experimental set-up for Irradiations at the Van de Graaff (VDG) 1 s shown. A $3 \mathrm{~cm}$ gas target was used so sigatficant energy 1088 of the beam occurred during its transit through the target. The scintillation counter was used to monitor any variations in the flux. The possible energy range for "clean" experiments was limited by the break-up energy of the tritoas on the gold beam stop or the stainless steel tubing. For beam energies above this critical energy, bignificant flux levels occurred at energies below the desired neutron energy; this was discovered by bombarding an empty gas target. Thus data were not taken for neutron energy dependence studies in the range of 6 to $14 \mathrm{HeV}$.

Dosimetry at the Van de Graaff was done with either indium (In) or aluminium (Al) folls. Indium was used for energies of $6 \mathrm{MeV}$ or below and aluminim was used for the $23.4 \mathrm{MeV}$ neutrons. Foll couraing was done by the Radiochemistry Group, particularly with the help of E. Bayhurst and F. Prestwood. The In ${ }^{115}\left(n, n^{\prime}\right)$ In ${ }^{175 m}$ and the $\mathrm{Al}^{27}(n, a) \mathrm{Na}^{24}$ reactions were used. For the metastable In ${ }^{115 \mathrm{~m}}$, the $0.335 \mathrm{MeV}$ gammas from the decay to stable In ${ }^{115}$ were counted in a low background $3 \times 3$ well-counter 


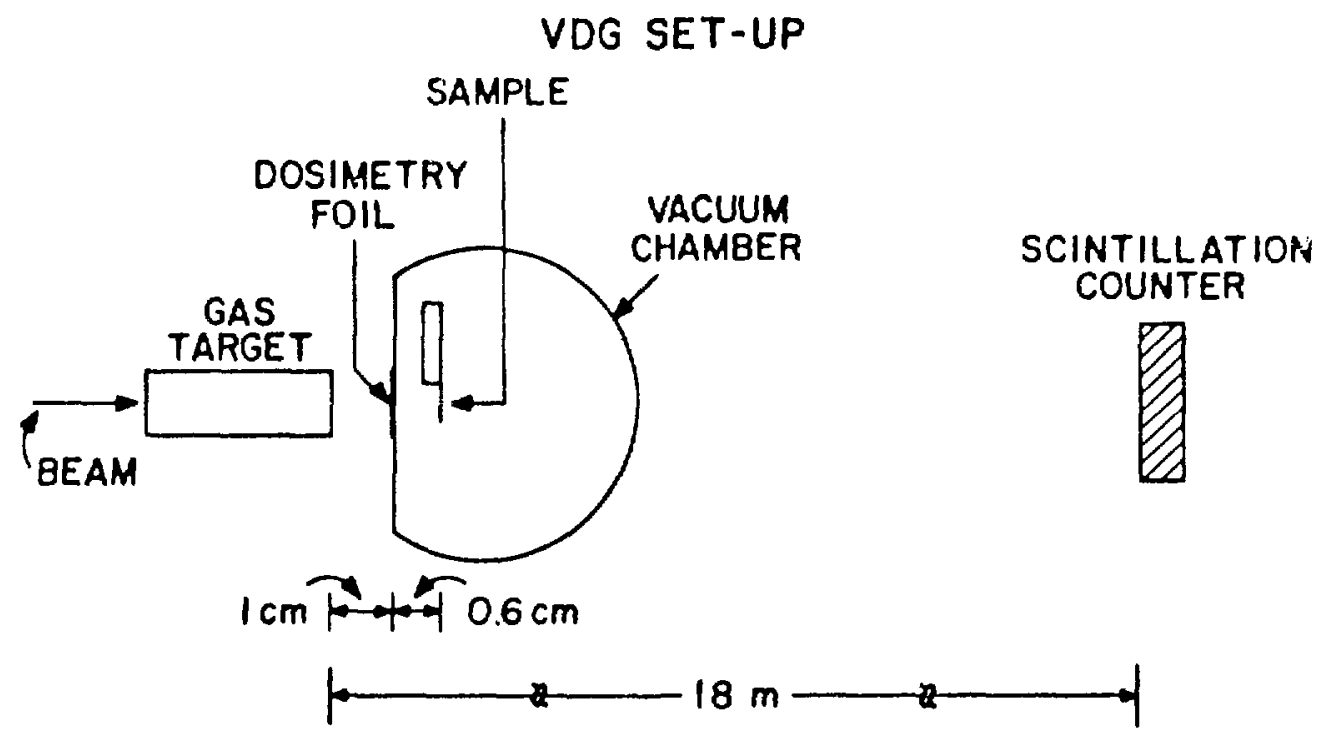

Figure 11: Experimental set-up for neutron irradiations at the Van de Graaff facility. A $3 \mathrm{~cm}$ gas target and indium or aluminum dosimetry foils were used. The scintillation counter was used for continuous monitoring of the flux. 
with a NaI Bcint11]ator and a multichannel analyzer. The $\mathrm{Na}^{24}$ was monitored by the decay to $\mathrm{Mg}^{24}$ through the emission of an electron followed by a $2.75 \mathrm{MeV}$ garma ray. The flux values, determined by gamma counting, at the dosimetry foll position were converted Into flux values at the sample position through use of a computer program called TIPI. This program, developed by the Radiochemistry Group, calculated the neutron energy and flux distribution at both the foll and sample posttion taking into account the gas target length and particle energy losses through the gas target. The average flux in the sample position was calculated with an accuracy of $10 \%$ primar1ly due to error in the measurement of distance (see appendix for flux calculation details). The deviation of the flux over the sample was found to be less than $4 \%$ from the average. Table 3 lists the experimental neutron energies and fluxes.

Experiments on the irradiation of gold by neutrons were done at the Mound Facility with the help of Dr. M. E. Anderson. Since the gold sample showed little response when irradiated with $2 \times 10^{8}$ neutrons $\mathrm{cm}^{-2} \mathrm{~s}^{-1}$ at Pajarito Site, a stronger neutron source was needed. A small KAMAN generator producing $14.3 \mathrm{MeV}$ neutrons at a source strength of $10^{11} \mathrm{~s}^{-1}$ at the sample was available at the Mound Facility. The sample was mounted in line with the beam and fluxes of $2 \times 10^{9} \mathrm{~cm}^{-2} \mathrm{~s}^{-1}$ at the sample produced visible results. Four copper pellets were placed in the dosimetry 
TABLE 3

EXPERIMENTAL NEUTRON ENERGIES AND FLUXES

\begin{tabular}{|c|c|c|c|}
\hline $\begin{array}{l}\text { Incoming } \\
\text { Particle } \\
\text { Energy } \\
\text { (MeV) }\end{array}$ & $\begin{array}{l}\text { Neut ron } \\
\text { Energy } \\
(\mathrm{MeV}) \\
\end{array}$ & Dosimetry & $\begin{array}{l}\text { Neutron } \\
\text { Flux } \\
\left(\mathrm{cm}^{-2} \mathrm{~s}^{-1}\right)\end{array}$ \\
\hline $4.4 \pm 0.2$ & $1.9 \pm 0.1$ & In foil. & $1.2 \pm 0.1 \times 10^{8}$ \\
\hline $6.9 \pm 0.1$ & $3.9 \pm 0.1$ & In foil & $1.4 \pm 0.1 \times 10^{8}$ \\
\hline $9.6 \pm 0.1$ & $5.9 \pm 0.1$ & In foil & $1.4 \pm 0.1 \times 10^{8}$ \\
\hline $0.30 \pm 0.03$ & $14.1 \pm 0.1$ & $\alpha$-monitor & $1.0 \pm 0.1 \times 10^{8}$ \\
\hline $6.0 \pm 0.2$ & $23.4 \pm 0.1$ & Al foil & $2.1 \pm 0.2 \times 10^{7}$ \\
\hline
\end{tabular}


foll position during irradiation. Two reactions, $\mathrm{Cu}^{63}(\mathrm{n}, \gamma) \mathrm{Cu}^{64}$ and $\mathrm{Cu}^{65}(\mathrm{n}, 2 \mathrm{n}) \mathrm{Cu}^{64}$, result in the production of $1.34 \mathrm{MeV}$ gammas and had to be taken into account when calculating fluxes. By noting the position of the four pellets and analyzing their flux values, it was found that the sample was not centered around the beam axis. Fortunately, for an essentially isotropic source, the conversion to flux at the sample was not difficult (Va74). Continuous monftoring of the flux was performed by use of a long counter (parafin around boron) set in the Irradiation room. Two possible sources of error during irradiations are transmutation reactions and scattered neutrons. Transmutation reactions might be important if the created impurities became additional pinning points on the dislocations. The atom fraction (af) of transmutations produced is given by the fluence times the cross section. Using copper for an example, the cross section for various reactions are: (1) $\mathrm{Cu}(n, 2 n) \mathrm{Cu}, 609 \mathrm{mb} ; \quad(2) \mathrm{Cu}(n, p) \mathrm{Ni}$, $79 \mathrm{mb}$; and (3) $\mathrm{Cu}(\mathrm{n}, \alpha) \mathrm{Co}, 36.7 \mathrm{mb}$. A typical irradiation was at a flux of $10^{8} \mathrm{~cm}^{-2} \mathrm{~s}^{-1}$ for at most 3600 seconds, yielding a total fluence of $3.6 \times 10^{11} \mathrm{~cm}^{-2}$. The total af of transmutations is $2.6 \times 10^{-13}$. Since the number of dislocated sites is roughly $2.4 \times 10^{-5}$, only a small fraction of transmutations would be on dislocations. Additionally, since the product of the $(n, 2 n)$ reaction is still a copper atom this reaction should not contribute to impurity pinning. Since the sample values are always monitored 
prior to irradiation and following post-irradiation annealing, a significant number of additional pinning points created in this manner would be noticed in the change of resonant frequency. Since no significant change in resonant frequency was noticed, it may be determined that this and any other mechanisms have not produced many additional pinning points.

The problem of scattered neutrons arises from the neutrons hitting the sample chamber, the copper cap, and other material near the sample. These neutrons could be scattered back through the sample and not through the dosimetry foil or be too low in energy to activate the foil. A Monte Carlo neutron scattering problem was set up by Luba Kmetyk to estimate the change in flux due to scattering. This code was run for 2 and $15 \mathrm{MeV}$ neutrons and the maximum number of additional neutrons due to scattering was $2 \%$ or less. This error can be folded into the flux error, but the largest error in the flux is still due to uncertainties in the distance measurements.

F. Defect Production

The defect production rate during irradiation is given by:

$$
\frac{d n}{d t}=\sigma_{d A} N_{A} \phi f
$$

where $\mathrm{n}$ is the concentration of Frenkel pairs, $\sigma_{d}$ is the displacement cross section, $N_{A}$ is the atomic number density, $\phi$ is the flux, and $f$ is the survival fraction. For electrons, $\sigma_{d}$ can be obtained 
from low temperature resistivity measurements of $\Delta \rho / \Delta \phi$ versus

electron energy. At $0.5 \mathrm{MeV}, \sigma_{d}$ for copper 185.9 barns (Ju73) assuming a Frenkel pair resistivity of $1.7 \times 10^{-4} \mathrm{~s}$-ca. Gold irradiated with $2 \mathrm{MeV}$ electrons has a 42 barn cross section (Ba64) using $2.2 \times 10^{-4} \Omega$-cm as the Frenkel pair resistivity (Lu75). Since irradiations were performed at room temperature and above, the number of defects surviving recombination processes at room temperature is estimated (from resistivity annealing studies) to be between $1 / 4$ and $1 / 3(\mathrm{Sc} 70)$. This is the survival fraction, $f$, for electrons. Thus for copper at $330 \mathrm{k}$, irradiated with $0.5 \mathrm{MeV}$ electrons at a flux of $10^{10} \mathrm{~cm}^{-2} \mathrm{~s}^{-1}$, the number of free Frenkel pairs produced is:

$$
\begin{aligned}
& \frac{\mathrm{dn}}{\mathrm{dt}}=\left(5.9 \times 10^{-24} \mathrm{~cm}^{2}\right)\left(8.45 \times 10^{22} \mathrm{~cm}^{-3}\right)\left(10^{10} \mathrm{~cm}^{-2} \mathrm{~s}^{-1}\right)(.25) \\
& \frac{\mathrm{dn}}{\mathrm{dt}}=1.3 \times 10^{9} \mathrm{~cm}^{-3} \mathrm{~s}^{-1} .
\end{aligned}
$$

This is the number of free interstitials available to pin dislocations.

For high energy neutrons, the defect production is not as simple and clusters can form directly in the cascade region. Thus the absolute value of $\sigma_{d}$ is difficult to obtain from electrical resistivity measurements. The displacement cross section can be calculated as discussed in the introduction, but it does not directly give the number of free interstitials produced above room temperature. The survival fraction or number of defects 
escaping the cascade is different from that for electrons. It Is expected that the same equation II-4I can be used with $\sigma_{d}$ replaced by the calculated NRT displacement cross section and $f$ for neutrons can be estimated from the data. The relation between the Frenkel pair production rate and the measured pinning rate is therefore needed.

The number of pinning points, $n_{d}$, added to a dislocation line is proportional to the number of free interstitials produced. This assumes that only interstitials are pinning points and that the number of pinners produced directly on a dislocation line is negligible. Simpson and Sosin (Si72a) have shown that

$$
\mathrm{n}_{\mathrm{d}} \propto \frac{(\Delta Y / Y)_{\mathrm{o}}}{(\Delta Y / Y)}-1 \equiv \mathrm{N}_{\mathrm{dy}}
$$

where $\Delta Y=Y_{e}-Y_{e}$ is the elastic cr fully pinned value of Young's modulus) and the subscript o refers to the pre-irradiation values. Since $Y \propto 1 / \tau^{2}$ (equation $I I-34$ ) and $\Delta Y / Y=\tau^{2} / \tau_{e}^{2}-1$ (equation II-35), then:

$$
N_{d y}=\frac{\tau_{0}^{2}-\tau^{2}}{\tau^{2}-\tau_{e}^{2}}
$$

The value of $\tau$ is measured prior to irradiation $\left(\tau_{0}\right)$, during irradiation $(\tau)$, and in the fully-pinned state ( $\tau_{\mathrm{e}}$; measured during electron irradiations only). The value of $\mathrm{N}_{\mathrm{dy}}$ can be calculated versus irradiation time; the change of the slope of 
$N_{d y}$ at the onset of irradiation ts proportional to the pinning rate. By equating pinning rates for neutron and electron experiments and using equation II-41 the survival fraction for neutrons can be estimated. 


\section{Experimental Results and Analysis}

\section{A. Introduction}

Electron Irradiations were done at the Wright State University electron accelerator. The beam energy was $0.5 \mathrm{MeV}$ for copper irradiations and $2.0 \mathrm{MeV}$ for gold irradiations. For flux dependence measurements in copper, the beam current was varied from 0.1 to $10 \mathrm{nA}$ and the sample held at $330 \mathrm{~K}$ during irradiations. This gave a flux variation of two orders of magnitude from $8.9 \times$ $10^{8} \mathrm{~cm}^{-2} \mathrm{~s}^{-1}$ to $9.5 \times 10^{10} \mathrm{~cm}^{-2} \mathrm{~s}^{-1}$. Temperature dependence measurements on copper were done at a constant flux (constant defect production rate) of $3 \times 10^{10} \mathrm{~cm}^{-2} \mathrm{~s}^{-1}$; the temperature was varied from $310 \mathrm{~K}$ to $410 \mathrm{~K}$ in $20 \mathrm{~K}$ steps. The gold sample was irradiated at $330 \mathrm{~K}$ with $1.2 \times 10^{10}$ and $4.0 \times 10^{10}$ electrons $\mathrm{cm}^{-2} \mathrm{~s}^{-1}$. No flux or temperature depe adence studies were done on the gold sample. In order to distinguish between electron and neutron irradiations, electron runs are herein denoted with no letter in front of the run number.

Neutron irradiations on the copper sample were done at Pajarito Site (P-nn runs) and at the Tandem Van de Graaff (VDG-nn runs) at the Los Alamos Scientific Laboratory. Flux and temperature dependences were studied at Pajarito Site with 14.1 MeV neutrons. With the sample held at $330 \mathrm{~K}$, the flux was varied from $2 \times 10^{7} \mathrm{~cm}^{-2} \mathrm{~s}^{-1}$ to $2 \times 10^{8} \mathrm{~cm}^{-2} \mathrm{~s}^{-1} ;$ and the temperature 
dependence was measured over a range of 310 to $410 \mathrm{~K}$ at a constant flux of $1 \times 10^{8} \mathrm{~cm}^{-2} \mathrm{~s}^{-1}$. Neutron energy dependence data were taken at the Van de Graaff where neutron energles of $2,4,6$, and 23.5 MeV were avaflable at the same beam line. Flux values ranged from $2 \times 10^{7} \mathrm{~cm}^{-2} \mathrm{~s}^{-1}$ for the $23.5 \mathrm{MeV}$ neutrons to roughly $1.5 \times 10^{8} \mathrm{~cm}^{-2} \mathrm{~s}^{-1}$ for the lower energies. The gold sample was Irradiated at the Mound Facllity w1th $14.3 \mathrm{MeV}$ neutrons at a flux of $2 \times 10^{9} \mathrm{~cm}^{-2} \mathrm{~s}^{-1}$ while again the sample temperature was held at $330 \mathrm{~K}$. Tables 4,5 , and 7 list the various run numbers, temperatures, fluxes, total irradiation times, neutron energies, and initial pinning rates for copper and gold irradiations.

The data taken during all runs consisted of the period and drive voltage measured as a function of time before and after irradiation was begun. Figure 12 is a typical example of the raw data ror the copper sample irradiated with $0.5 \mathrm{MeV}$ electrons at $3.2 \times 10^{10} \mathrm{~cm}^{-2} \mathrm{~s}^{-1}$ (run 2). After the irradiation was begun, a definite change in the slope of the period and drive voltage occurred. The period decreased monotonically with time, while the drive voltage increased to sote peak value. In Figure 13, the data of Figure 12 has been reduced according to equations II-33 for the decrement and II-43 for $\mathbb{N}_{\mathrm{dy}}$. The $\mathrm{N}_{\mathrm{dy}}$ data versus time was fitted to a quadratic equation in time $\left(a+b t+c t^{2}\right)$ as expected from the equations given by keefer et al. (Ke65) for the dependence of $\triangle Y / Y$ on flux for a one dislocation model. The data 
TABLE 4

$0.5 \mathrm{MeV}$ ELECTRON IRRADIATIONS OF COPPER

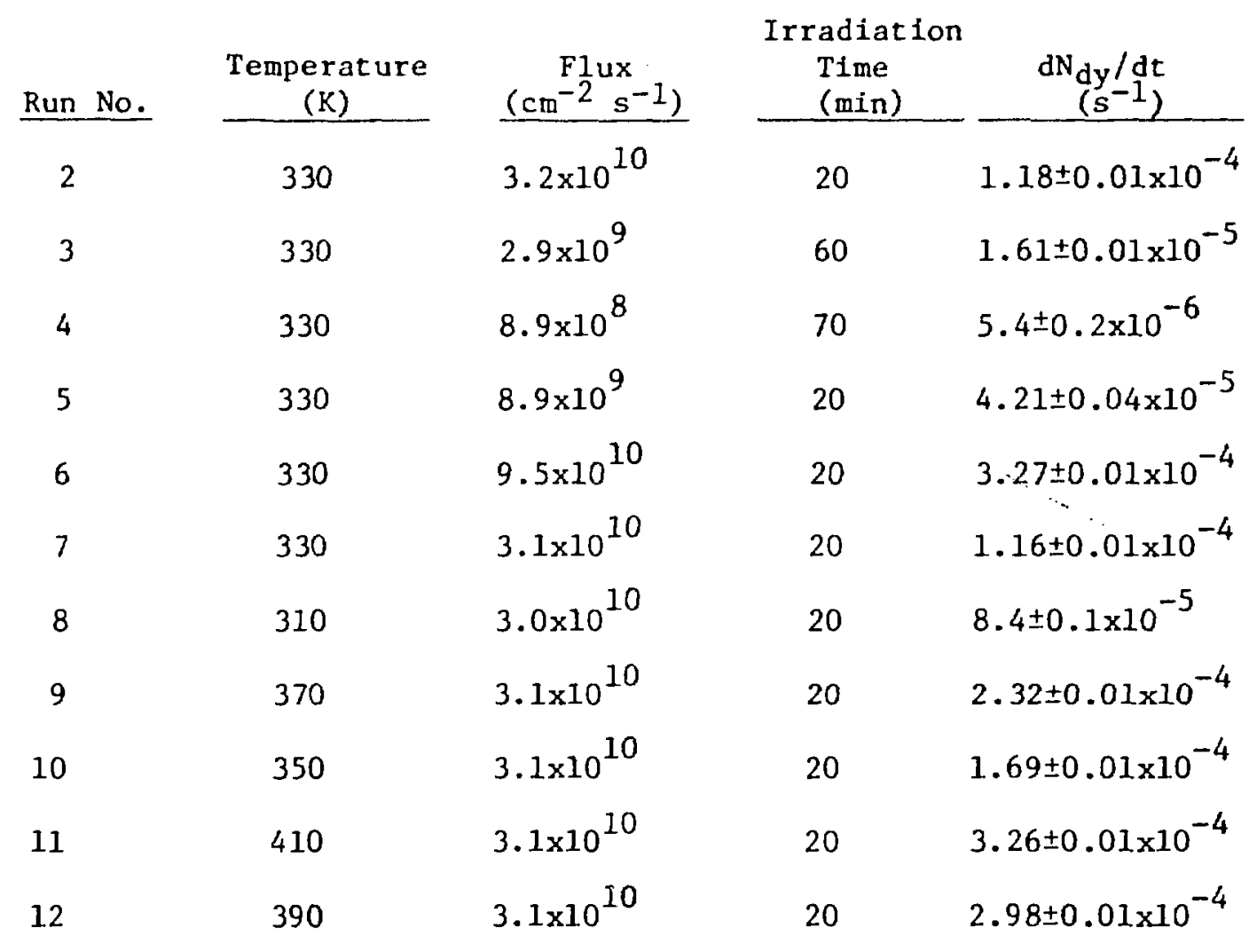


TABLE 5

NEUTRON IRRADIATIONS OF COPPER

\begin{tabular}{|c|c|c|c|c|c|}
\hline Run No. & $\begin{array}{c}\text { Temperature } \\
\text { (K) } \\
\end{array}$ & $\begin{array}{l}\text { Neutron } \\
\text { Energy } \\
\text { (MeV) } \\
\end{array}$ & $\left(\mathrm{cm}^{-2} \mathrm{~s}^{-1}\right)$ & $\begin{array}{l}\text { Irradiatio } \\
\text { Time } \\
\text { (min) }\end{array}$ & $\left.\mathrm{dN}_{\mathrm{dy}} / \mathrm{s}^{-1}\right)$ \\
\hline P-8 & 330 & $14.1 \pm 0.1$ & $2.1 \pm 0.2 \times 10^{8}$ & 57 & $2.7 \pm 0.2 \times 10^{-5}$ \\
\hline P-9 & 400 & $14.1 \pm 0.1$ & $2.4 \pm 0.2 \times 10^{8}$ & 27 & $1.1 \pm 0.1 \times 10^{-4}$ \\
\hline P-11 & 330 & $14.1 \pm 0.1$ & $1.6 \pm 0.2 \times 10^{7}$ & 178 & $2.5 \pm 0.1 \times 10^{-6}$ \\
\hline P-12 & 330 & $14.1 \pm 0.1$ & $7.2 \pm 0.7 \times 10^{7}$ & 72 & $9.1 \pm 0.8 \times 10^{-6}$ \\
\hline P-13 & 330 & $14.1 \pm 0.1$ & $4.7 \pm 0.5 \times 10^{7}$ & 62 & $6.1 \pm 0.5 \times 10^{-6}$ \\
\hline$P-14$ & 330 & $14.1 \pm 0.1$ & $2.0 \pm 0.2 \times 10^{7}$ & 141 & $2.8 \pm 0.2 \times 10^{-6}$ \\
\hline$P-15$ & 330 & $14.1 \pm 0.1$ & $3.0 \pm 0.3 \times 10^{7}$ & 86 & $4.6 \pm 0.4 \times 10^{-6}$ \\
\hline$P-17$ & 330 & $14.1 \pm 0.1$ & $1.3 \pm 0.1 \times 10^{8}$ & 45 & $1.4 \pm 0.1 \times 10^{-5}$ \\
\hline P-18 & 390 & $14.1 \pm 0.1$ & $1.3 \pm 0.1 \times 10^{8}$ & 20 & $3.6 \pm 0.1 \times 10^{-5}$ \\
\hline P-19 & 310 & $14.1 \pm 0.1$ & $1.2 \pm 0.1 \times 10^{8}$ & 93 & $9.6 \pm 0.5 \times 10^{-6}$ \\
\hline$P-20$ & 370 & $14.1 \pm 0.1$ & $1.3 \pm 0.1 \times 10^{8}$ & 31 & $3.1 \pm 0.2 \times 10^{-5}$ \\
\hline$P-21$ & 350 & $14.1 \pm 0.1$ & $1.2 \pm 0.1 \times 10^{8}$ & 41 & $2.1 \pm 0.1 \times 10^{-5}$ \\
\hline$P-22$ & 330 & $14.1 \pm 0.1$ & $1.2 \pm 0.1 \times 10^{8}$ & 72 & $1.4 \pm 0.1 \times 10^{-5}$ \\
\hline$P-23$ & 410 & $14.1 \pm 0.1$ & $1.3 \pm 0.1 \times 10^{8}$ & 20 & $4.4 \pm 0.3 \times 10^{-5}$ \\
\hline $\mathrm{P}-28$ & 330 & $14.1 \pm 0.1$ & $2.3 \pm 0.2 \times 10^{8}$ & 31 & $2.2 \pm 0.1 \times 10^{-5}$ \\
\hline VDG-25 & 330 & $23.4 \pm 0.1$ & $2.1 \pm 0.2 \times 10^{7}$ & 104 & $3.5 \pm 0.1 \times 10^{-6}$ \\
\hline VDG-26 & 330 & $1.9 \pm 0.1$ & $1.2 \pm 0.1 \times 10^{8}$ & 60 & $6.2 \pm 0.1 \times 10^{-6}$ \\
\hline VDG-27 & 330 & $3.9 \pm 0.1$ & $1.4 \pm 0.1 \times 10^{8}$ & 57 & $9.3 \pm 0.6 \times 10^{-6}$ \\
\hline VDG-28 & 330 & $5.9 \pm 0.1$ & $1.4 \div 0.1 \times 10^{8}$ & 45 & $1.04 \pm 0.01 \times 10^{-5}$ \\
\hline
\end{tabular}




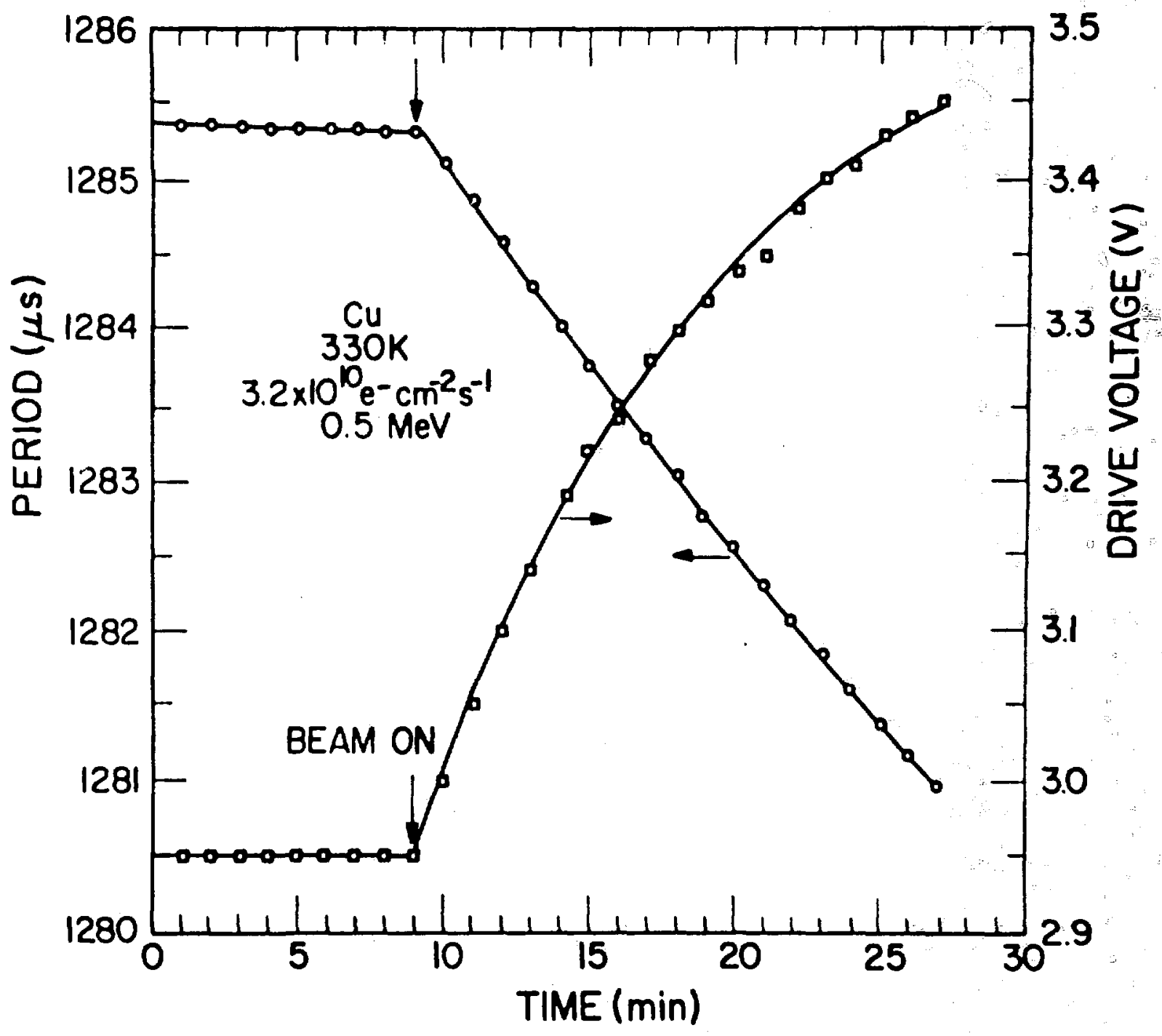

Figure 12: A typical example of the raw data versus time for a $0.5 \mathrm{MeV}$ electron irradiation of the copper sample held at $330 \mathrm{~K}$. The irradiation was begun at the beam-on pointer. 


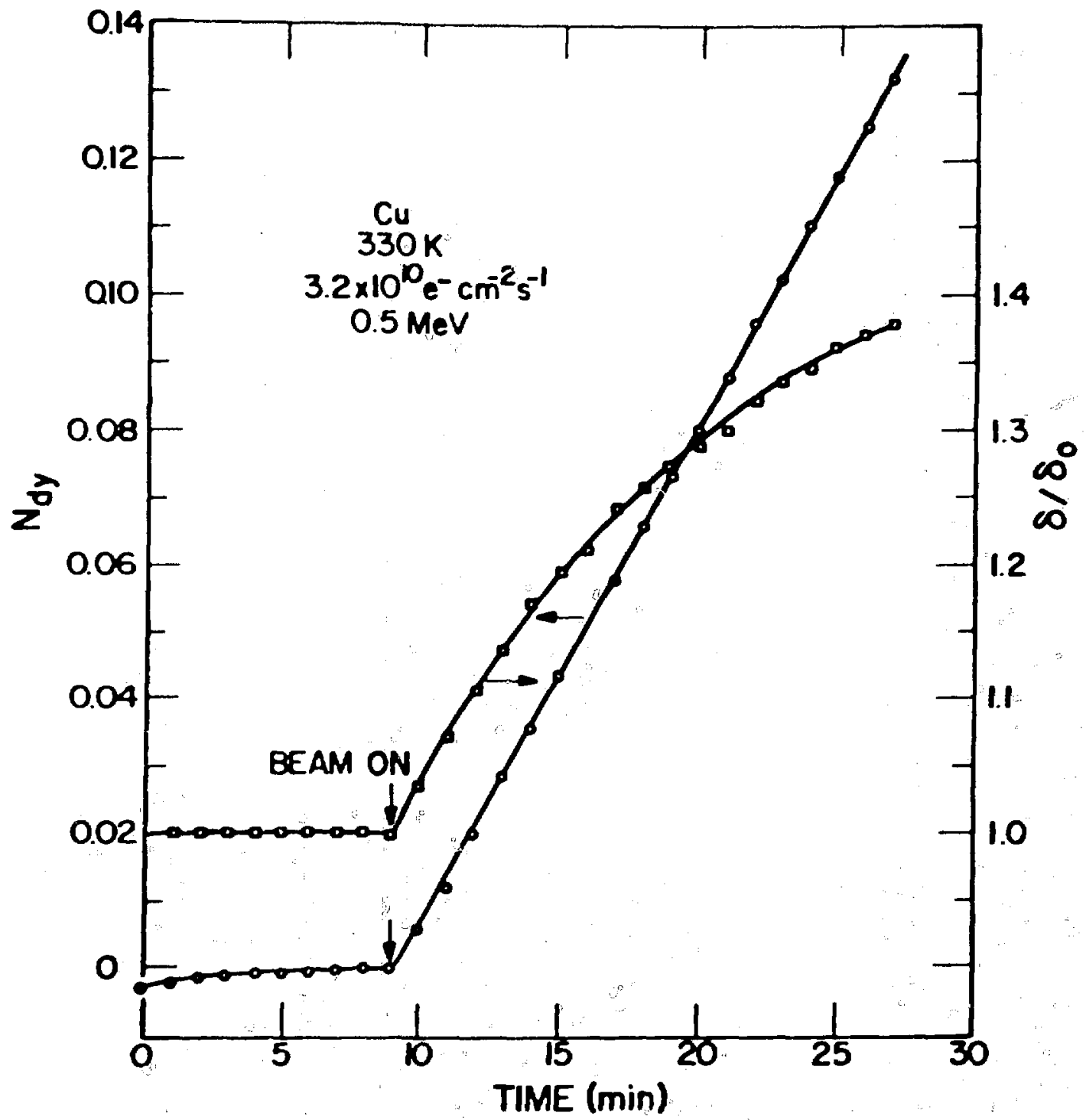

Figure 13: The reduced data from the points recorded in

Figure 12 by using equations $I I-33$ and II-43. 
prior to lrradiation and during irradiation were fitted separately; thus the Initlal pinning rate due to radiation induced defects was the charge 1n slope at the onset of irradiation. The values of the Initfal pinning rates are given in Tables 4 and 5 for the copper data and Table 7 for gold irradiations.

\section{B. Flux Dependence in Copper}

The kinetics of accumulation of defects on a dislocation line are based on the following equations (Si73):

$$
\begin{aligned}
& \frac{d n}{d t}=P-n D \Lambda \\
& \frac{d n_{d}}{d t}=n D(1-\Delta)-K_{d} n_{d}+K_{N} N \\
& \frac{d N}{d t}=n D \Delta+K_{d} n_{d}-K_{N} N
\end{aligned}
$$

where $n$ is the concentration per unit volume of interstitials in the lattice (not at dislccations) at time $t, P$ is the creation rate of defects in the lattice (proportional to the flux), D is the temperature-dependent diffusion coefficient, $\Lambda$ is the areal density of dislocations of average length $1, \mathrm{n}_{\mathrm{d}}$ is the number of point defects on dislocations excluding a short segment $\Delta$ at the ends associated with nodes, $N$ is the number of interstitials at nodal points, $\mathrm{K}_{\mathrm{d}}$ is the jump rate of interstitials along the dislocation line and not at nodes, and $k_{N}$ is the rate at which point defects return to the dislocation lines from nodal points. 
It 1s assumed in writing these equations that (1) interstitials are the moblla defect, (2) the dislocations are pinned only at nodes prior to frradlation, (3) no significant number of defects are created on the dislocation lines, and (4) no defect-defect Interactions occur on the dislocation line. The solution of these equations for extended 1rradlation times 1s:

$$
n_{d}=P_{d D K}{ }^{-1} t
$$

with the additional assumption that $\mathrm{K}_{\mathrm{N}} \ll \mathrm{K}_{\mathrm{d}}$. For a constant temperature ( 1 and $\mathrm{K}_{\mathrm{d}}$ constant), the time rate of charpes of $\mathrm{n}_{\mathrm{d}}$ is linearly dependerit on the creation rate or equivalently the flux. The flux dependence of the pinning $x$ ate in copper was measured at $330 \mathrm{~K}$ over two orders of magnitude in electron flux and one order of magnitude in neutron flux. Figures 14 and 15 show the variation of $\mathrm{N}_{\mathrm{dy}}$ with time for several electron and neutron irradiations, respectively. As the flux increased, the rate of change of $N_{d y}$ increased for both electron and neutron irradiations. The initial radiation induced pinning rate as a function of electron and neutron flux is shown in Figure 16. The electron and neutron data points were separately fit to the following equation:

$$
\frac{d N}{d t}=b \phi^{a}
$$

Both sets of data showed a non-linear dependence on the flux and 


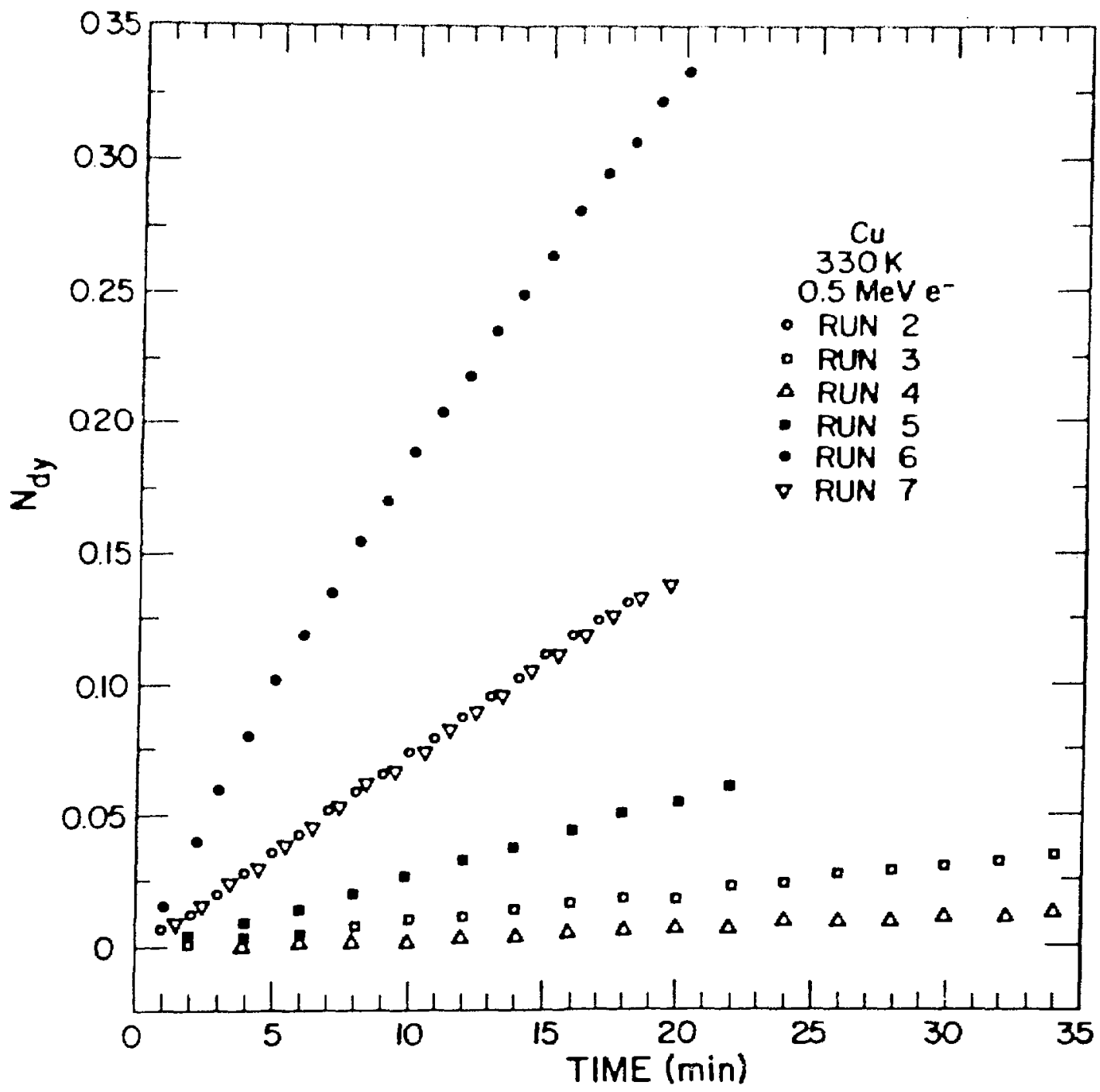

Figure 14: $\mathrm{N}_{\mathrm{dy}}$ versus time for the flux dependence studies of electron irradiation on copper. Zero time is the onset of irradiation. Run numbers refers to values given in Table 4. 


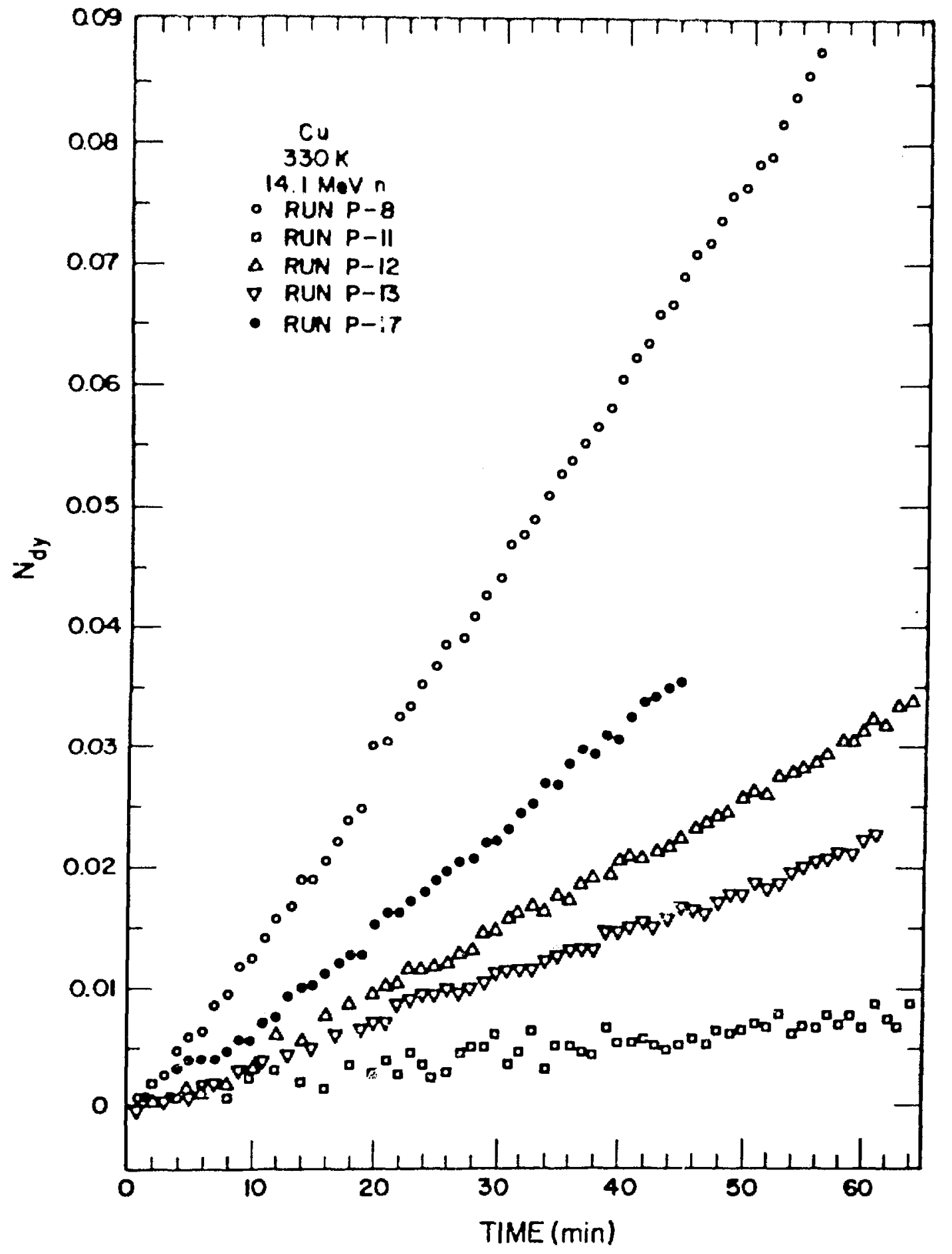

Figure 15: $\mathbb{N}_{d y}$ versus time for neutron flux dependence runs. Zero time is marked from the onset of irradiation. Run numbers refer to Table 5 . 


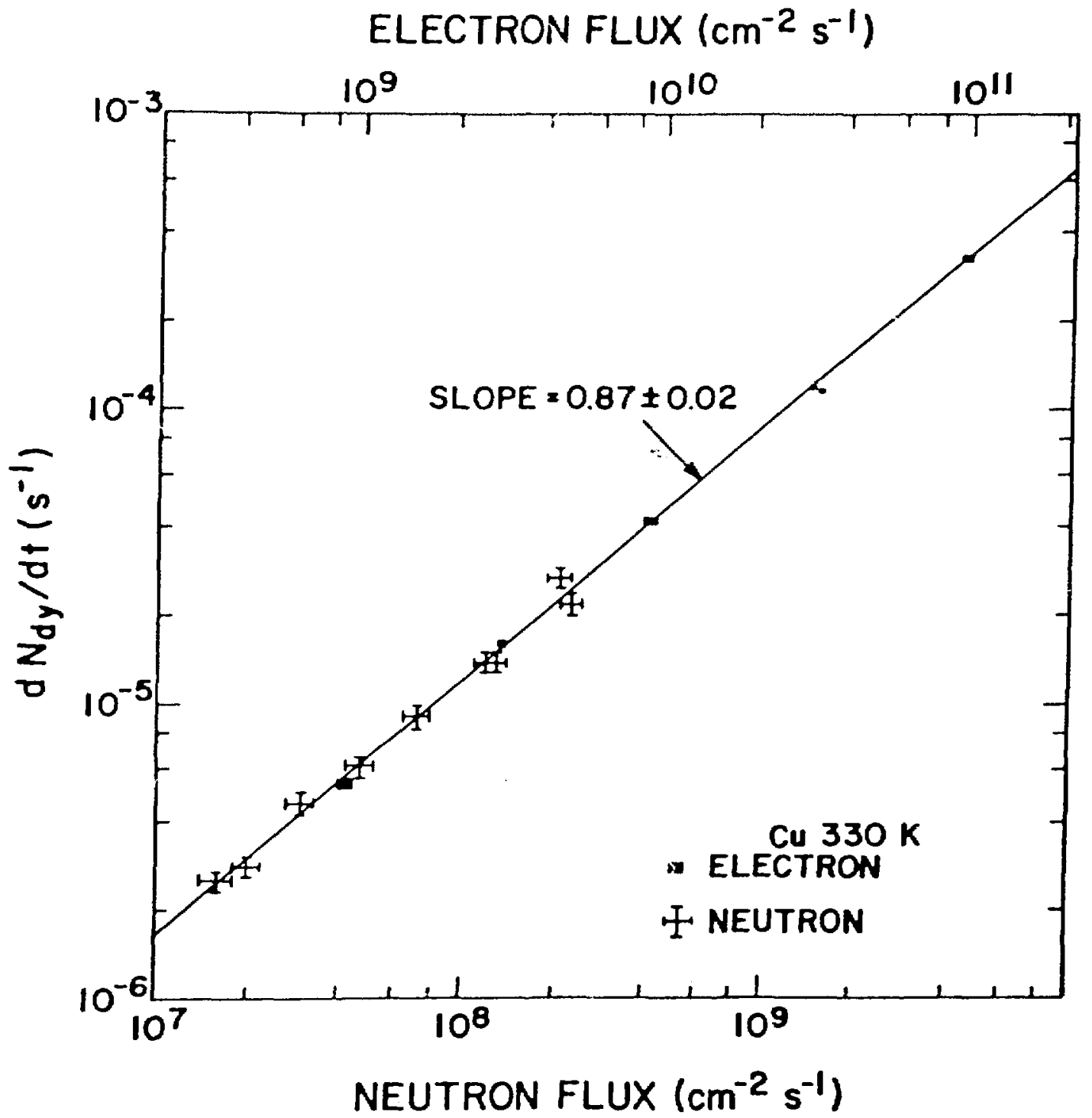

Figure 16: Initial pinning rate versus electron and neutron flux. Contrary to the prediction of equation III-47, the slope of the straight line fit is not 1 but 0.87 . Both electron and neutron data points exhibit the same flux dependence. 
the value of a in both rases was 0.87 . AJ alternat 1 ve and 1 mportant way to examine the data was to geale che electron data to the neutron daca so that al] the data fit on a comon sitralght Ine as, shown in figure 16. Runs $2, P-13$, and $p-15$ had close inftlal pinning rates so that overlap of the data caisted. Agatn, as expected, the best fit vilue was a $=5.87 \pm 0.02$. In addition, the factor uscd to sicale the relectron flux to the reutron flux that produced the samp inital pluning rate was found to the $21.7 \pm 0.7$ (where the uncertainty was determined by varying the reduced $x^{2}$ from its minimum of 1.00 to a valur of 1.05 ).

\section{Temperature Hependenec in copper}

Equation III-47, repeated below, can be used to analyze the temperature dependence of the pinning rate at constant flux:

$$
n_{\mathrm{d}}=P I D K_{\mathrm{d}}^{-1} \mathrm{t}
$$

Now $D$ and $k_{d}$ are temperature dependent diffucior coefficients. If $\mathrm{H}_{\mathrm{m}}$ is the migration enthalpy of an interstitial to the dislocation lines and $\mathrm{H}_{\mathrm{d}}$ is the migration enthalpy of an interstitial along the dislocation Iine, then:

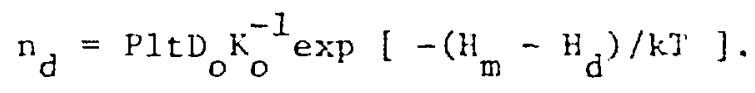

The slope of the logarithm of the initial pinning rate versus inverse temperature gives the value of $\mathrm{H}_{\mathrm{m}}-\mathrm{H}_{\mathrm{d}}$. 
The terperature dependence of the plondng rate wiss ratesured at $3.3 \times 10^{10}$ electrons $\mathrm{cm}^{-2} \mathrm{~s}^{-1}$ and $1.3 \times 10^{8}$ neutrons $\mathrm{cm}^{-2} \mathrm{~s}^{-1}$ over the range 310 to $410 \mathrm{~K}$. Flgures 17 and 18 show the vartation of Nidy versus time ior several ejectron and neutron trradiations. As the cemperature was incrased from $310 \mathrm{~K}$ in $20 \mathrm{k}$ seps, the Initlal pinning rate increased wat il a temperature of $410 \mathrm{k}$ was reached. At $410 \mathrm{k}$ signdficant depinntng of the dislocation lines occurred so that equation III-49 wast no longer valid. The data, thertiore, was only fit to $390 \mathrm{~K}$ in both electron and neutron cases. In Figure 19, the logarithm of dN dy /de versus 1000/T is shown for both electron and neut ron irradiations. The electron data has been scaled according to couation III-48 with a $=0.87$ so it overlapped the neutron data by using the scale factor 21.7 . Separatedy, ihe clectron data poines yielded a value of $\mathrm{H}_{\mathrm{m}}-\mathrm{H}_{\mathrm{d}}$ of $0.17 \pm 0.01 \mathrm{eV}$ and the neutron data gave $0.18 \pm 0.02 \mathrm{cV}$ over the temperature range of 310 to $390 \mathrm{~K}$. The combined data as shown in Figure 19 gave a composite value of $0.17 \pm 0.02 \mathrm{eV}$ for $\mathrm{H}_{\mathrm{DS}}-\mathrm{H}_{\mathrm{d}}$.

i). Pieutron Energy Dependence in Copper

The neutron energy dependence of the pinning rate should icllow one of the three models discussed in section $A$ of the introduction: 


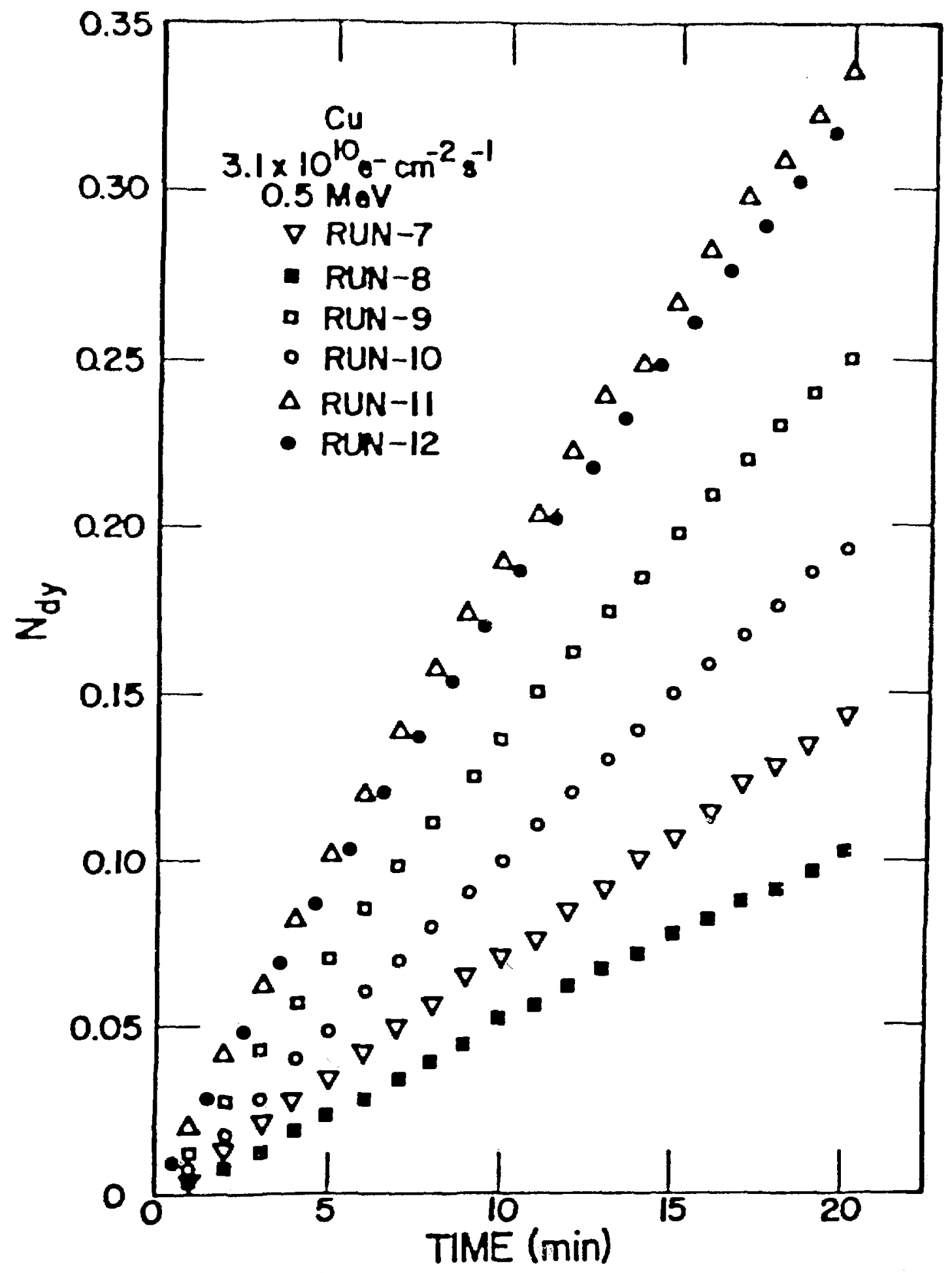

Figure 17: $\mathrm{N}_{\mathrm{dy}}$ versus time for electron temperature dependence runs. Zero time is marked from the onset of irradiation. Run numbers refer to Table 4. 


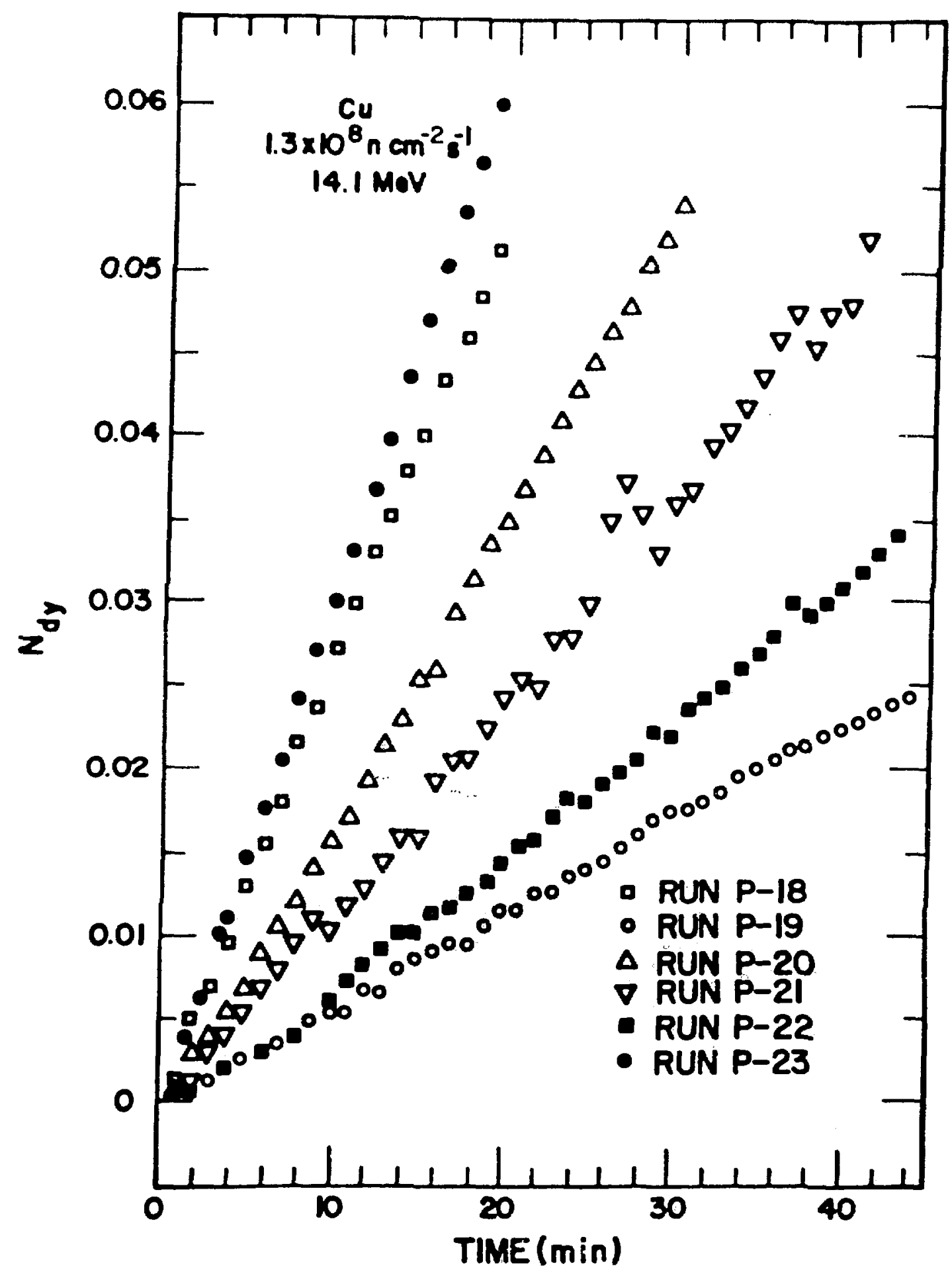

Figure 18: $\mathrm{N}_{\mathrm{dy}}$ versus time for neutron temperature dependence runs. Zero time coincj.des with the onset of irradiation. Run numbers refer to Table 5. 


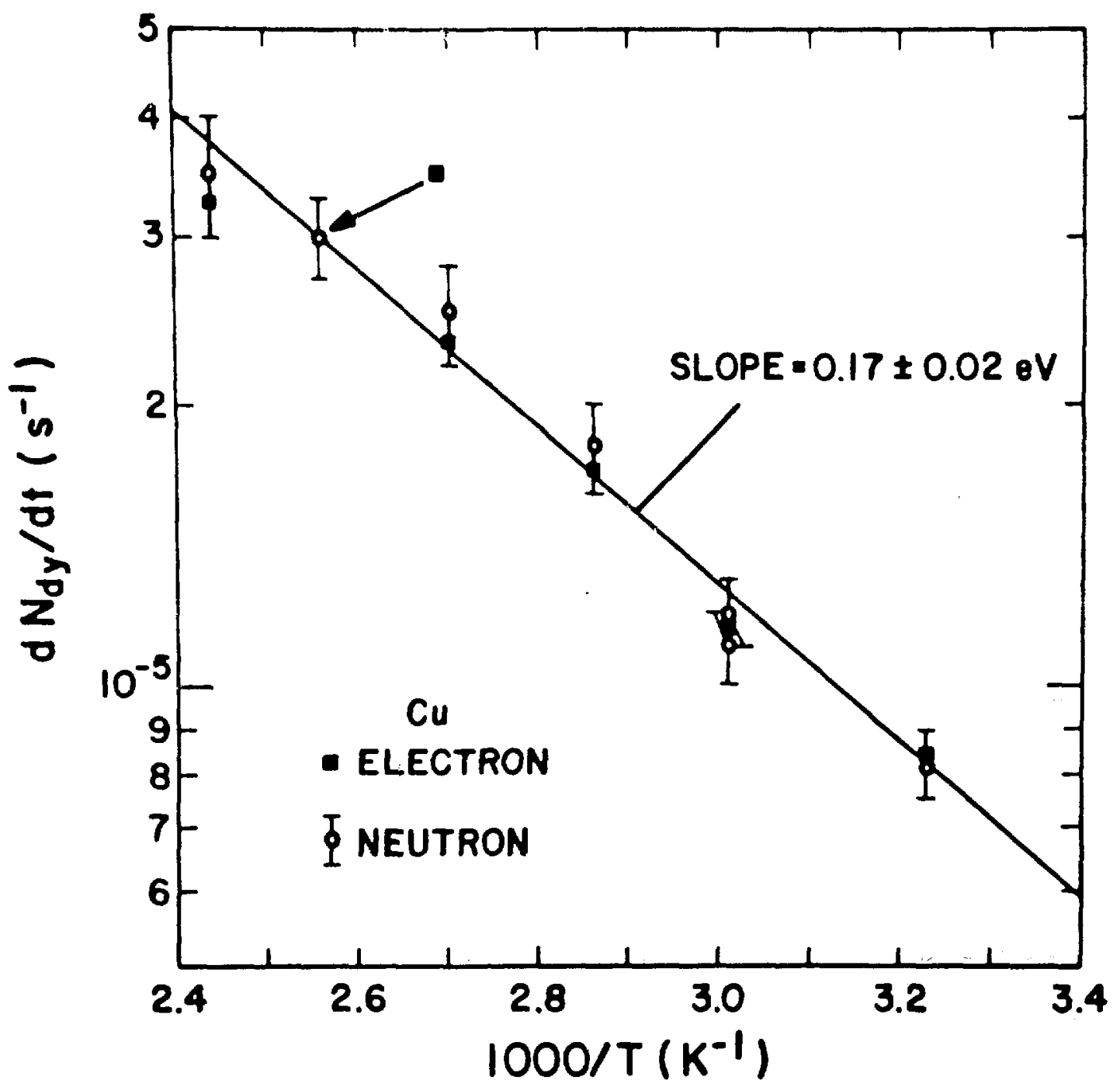

Figure 19: Initial pinning race versus temperature for both electron and neutron irradiations. The slope of the line gives the value of $\mathrm{H}_{\mathrm{m}}-\mathrm{H}_{\mathrm{d}}$ of equation III-49. The electron data has been scaled by using equation III-48 with $a=0.87$ and $\phi_{1} / \phi_{2}=21.7$ 


$$
\begin{aligned}
g(T) & =K T_{d a m} / 2 E_{d} \\
& =A\left[1-B I n\left(T_{d a m}\right)\right] T_{d a m} \\
& =T_{\text {dam }} /\left(a+B T_{\text {dam }}\right)
\end{aligned}
$$

where the constants are defined for copper in the introduction and $T_{\text {dam }}$ is given by equation $1-5$. The above statement is true if the fraction of free interstitials escaping from the displacement cascade is nearly constant with increaslng primary recoll energy, for the range of recoll energles important in this experiment. An argument for the validity of this assumption w111 be presented in the discussion of the free interstitlal production cross sections. As pointed out earlier, the difference between the functions of damage energy given by $\mathrm{I}-6$ and $\mathrm{I}-7$ (NRT and RT-b models) is not significant when the slope of the displacement cross section is examined over the energy range covered in this experiment. However, the difference between either of these models and the energy dependence of equation I-8 (RT-3 model) should allow differentiation among the theories. Doran's residual defect model (RDM) given by equation I-9 is also very similar to the RT-b model of equation I-7. The ratio of the displacement cross section at $2 \mathrm{MeV}$ to that at $20 \mathrm{MeV}$ for copper is $0.25,0.27$, and 0.28 for the NRT, RT-b, and RDM expressions while for the RT-3 the ratio is 0.64 .

The energy dependence was measured by experiments with 2 , 
4, 6, 14, and $23.5 \mathrm{MCV}$ neutrons. F1gures 20 and 21 show the raw and reduced data, respectively for run VDG-28 with $6.0 \mathrm{MeV}$ neutrons at a flux of $1.4 \times 10^{8} \mathrm{~cm}^{-2} \mathrm{~s}^{-1}$. Agaln the drive voltage and the decrement increased with the while the period decreased ( $N_{\text {dy }}$ increased). Figure 22 shows the values of $N_{d y}$ versus $t+m e$ for the neutron energy dependence runs done at the Van de Graaff. Since the runs were not done at the same $f 1 x x$, the data in Figure 22 cannot be directly compared. The important parameter in this measurement was the number of neutrons $\mathrm{cm}^{-2} \mathrm{~s}^{-1}$ at a given neutron energy it took to produce a given rate of property change. In this case, equation III-48 was used to determine from the data the flux at each energy that would produce a selected pinning rate:

$$
\frac{\phi_{1}}{\Phi_{2}}=\left(\frac{s_{1}}{s_{2}}\right)^{1 / a}
$$

where $\phi$ refers to the flux and $s$ to the initiai pinning rate. Thus $s_{2}$ and $\phi_{2}$ were the measured initial pinning rate and flux at a given energy and $\phi_{1}$ is the flux calculated to produce the standard pinning rate $5_{1}$. Table 6 gives the flux versus neutron energy. for a pinning rate of $2 \times 10^{-5} \mathrm{~s}^{-1}$. The effect per neutron was found by dividing the chosen pinning rate by the calculated neutron flux. These values are given in the last colum of Table 6. It can be seen that the number of pinning points per neutron increases with increasing neutron energy. In Figure 23, the data 


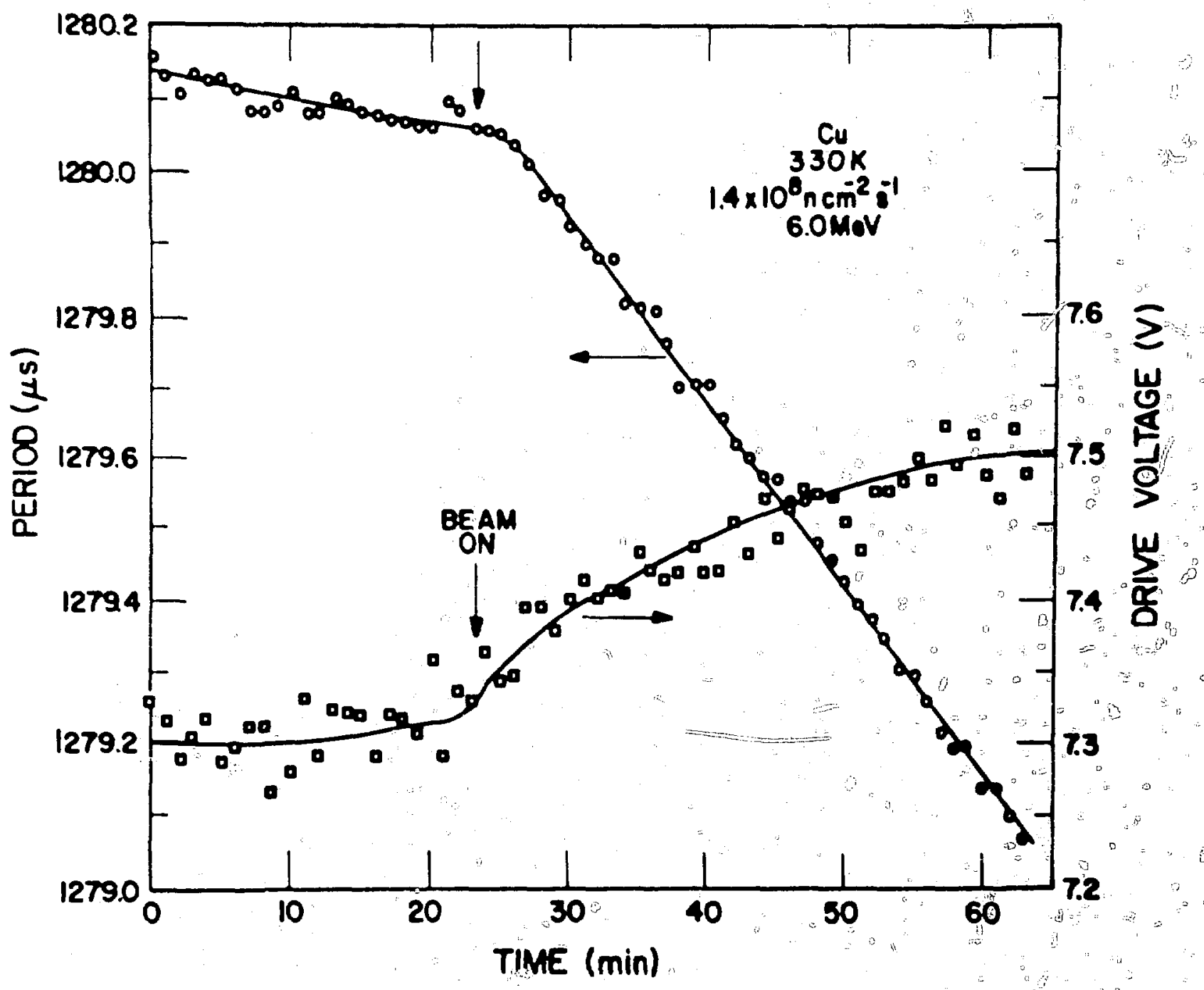

Figure 20: An example of ráw data versus time for a neutron 1rradiation. The data corresponds to run VDG-28 with 60 $\mathrm{MeV}$ neutrons at a flux of $1.4 \times 10^{8} \mathrm{~cm}^{-2} \mathrm{~s}^{-1}$. The sample bas at $330 \mathrm{~K}$. Note the slight delay in any detectable change in the period after the irradiation was begun. 


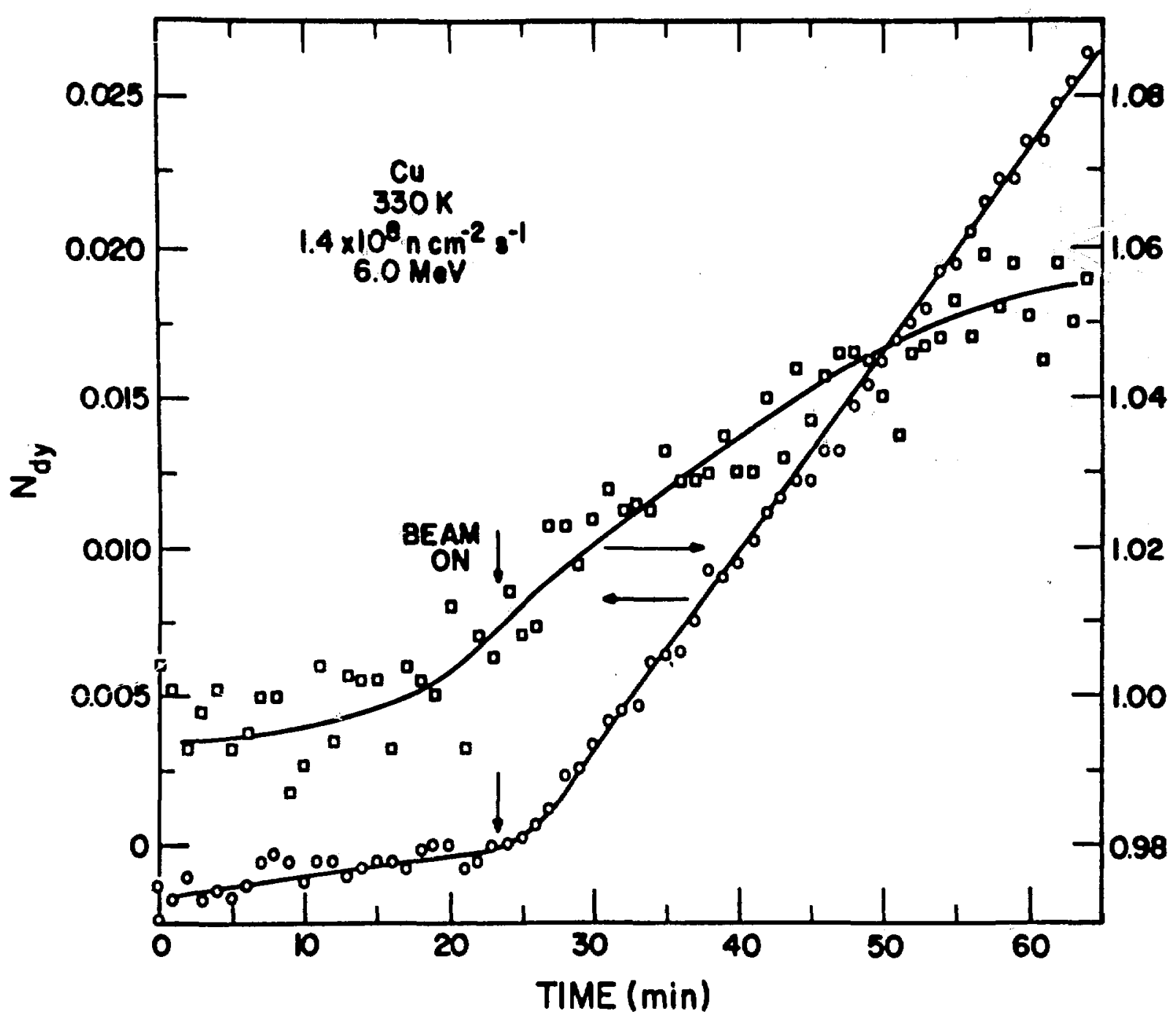

Figure 21: The reduced data from points recorded in

Figure 20 by using equations II-33 and II-43. 


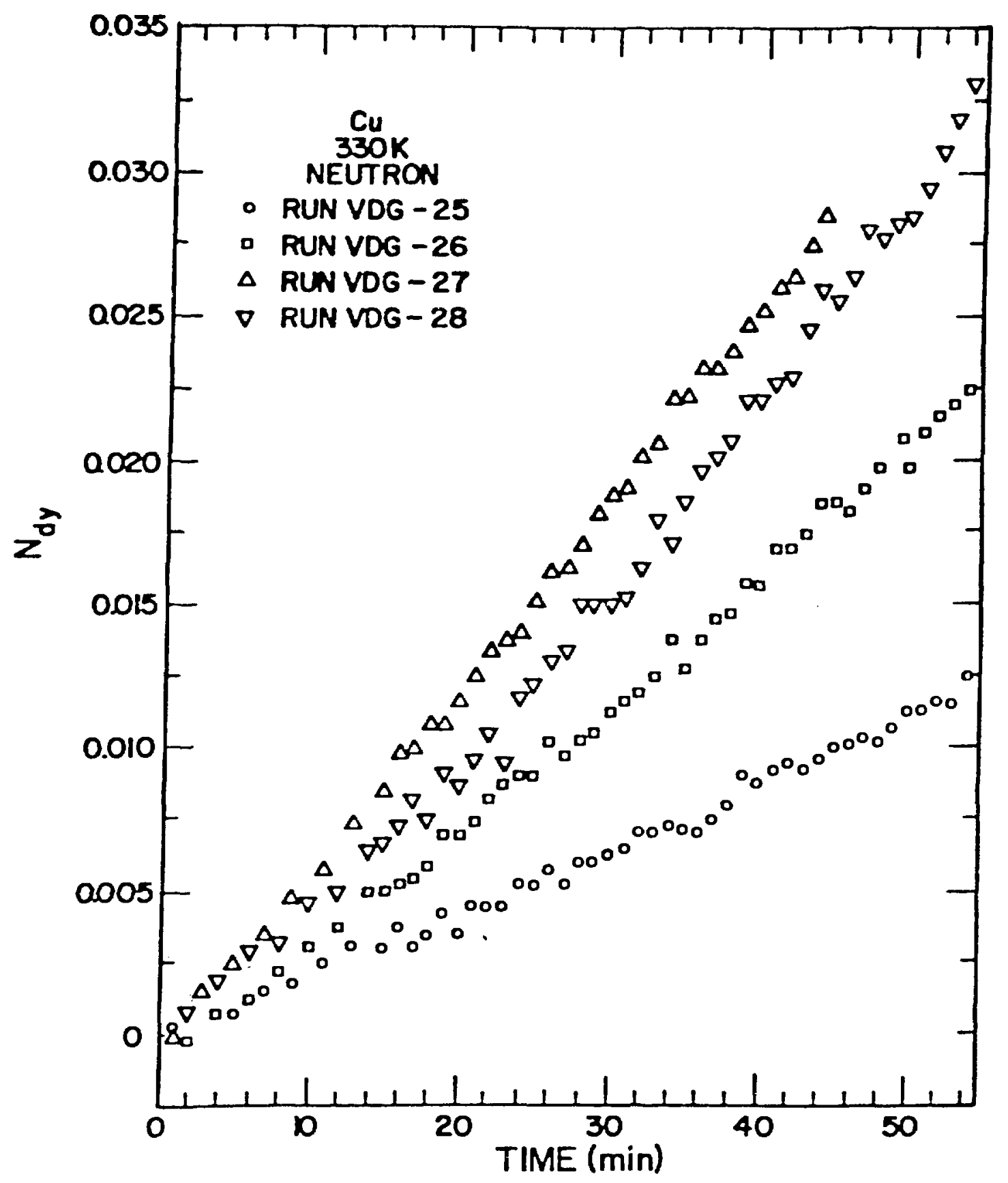

Figure 22: $\mathrm{N}_{\mathrm{dy}}$ versus time for the neutron energy dependence data on copper. Zero time is the onset of irradiation. Runs cannot be directly compared because of the different flux values used. Numbers refer to runs listed in Table 5. 
TABLE 6

PINNING POINTS PER NEUTRON IN COPPER ${ }^{1}$

\begin{tabular}{|c|c|c|c|c|c|}
\hline Run No. & $\begin{array}{c}\text { Neutron } \\
\text { Energy } \\
(\mathrm{MeV}) \\
\end{array}$ & $\left.\mathrm{dN}_{(\mathrm{dy} / \mathrm{dt}}-1\right)$ & $\left(c m^{-2} s^{-1}\right)$ & $\begin{array}{c}\mathrm{dN}_{\mathrm{dy}} / \mathrm{d} \Phi \\
\left(\mathrm{cm}^{2}\right)\end{array}$ & $\begin{array}{l}\text { Relative } \\
\text { PInning }\end{array}$ \\
\hline$V D G-26$ & $1.9 \pm 0.1$ & $2.0 \times 10^{-5}$ & $4.4 \pm 0.5 \times 10^{8}$ & $4.5 \pm 0.5 \times 10^{-14}$ & $0.45 \pm 0.05$ \\
\hline VDG-27 & $3.9 \pm 0.1$ & $2.0 \times 10^{-5}$ & $3.4 \pm 0.5 \times 10^{8}$ & $5.8 \pm 0.8 \times 10^{-14}$ & $0.58 \pm 0.08$ \\
\hline$\sqrt{2} C-28$ & $5.9 \pm 0.1$ & $2.0 \times 10^{-5}$ & $2.9 \pm 0.3 \times 10^{8}$ & $6.9 \pm 0.8 \times 10^{-14}$ & $0.69 \pm 0.08$ \\
\hline$P-17$ & $14.1 \pm 0.1$ & $2.0 \times 10^{-5}$ & $2.0 \pm 0.4 \times 10^{7}$ & $1.0 \pm 0.2 \times 10^{-13}$ & $1.0 \pm 0.2$ \\
\hline VDG -25 & $23.4 \pm 0.1$ & $2.0 \times 10^{-5}$ & $1.5 \pm 0.2 \times 10^{7}$ & $1.3 \pm 0.2 \times 10^{-13}$ & $1.3 \pm 0.2$ \\
\hline
\end{tabular}




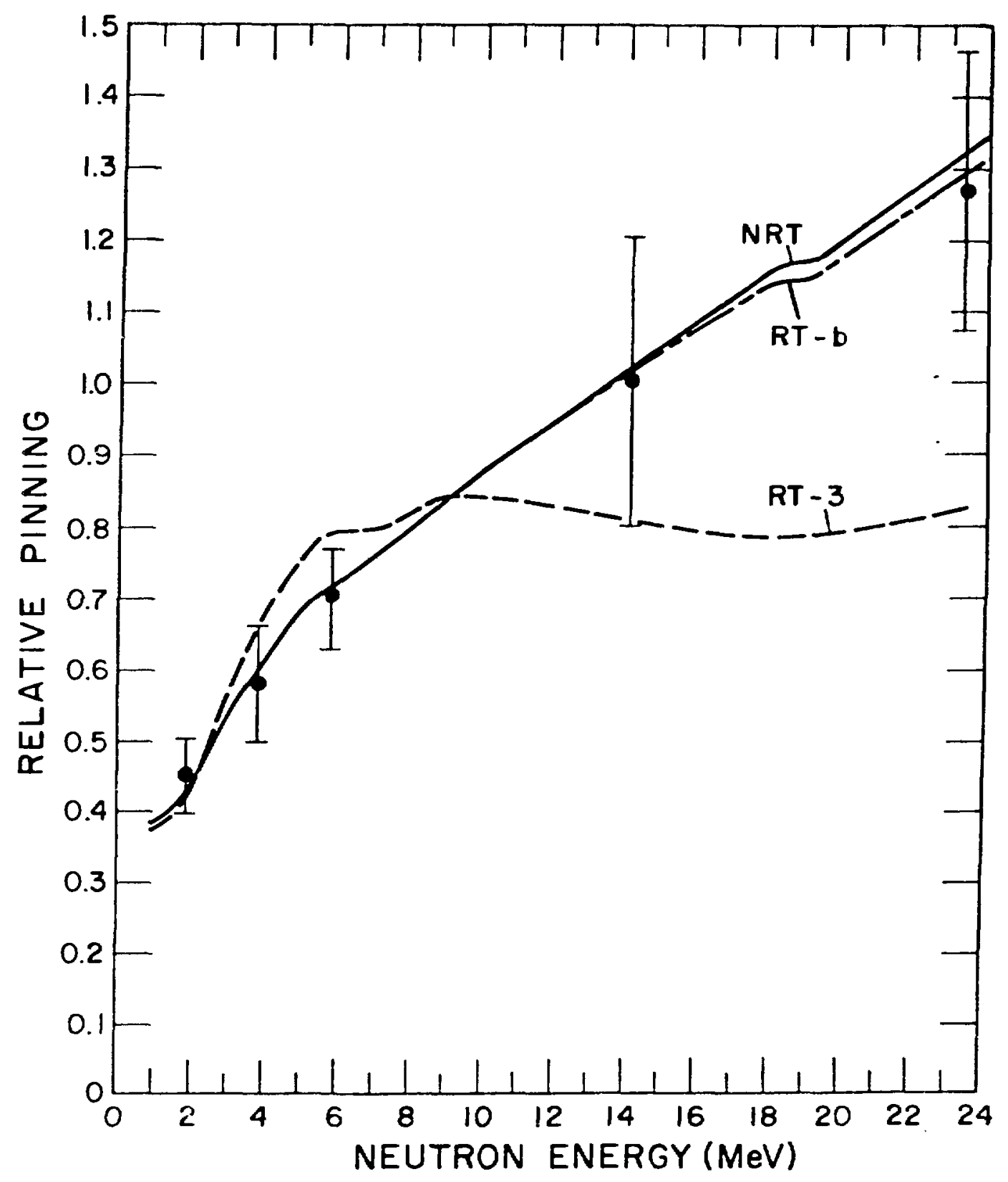

Figure 23: The relative pinning (see Table 6) versus neutron energy for $2,4,6,14$, and $23.5 \mathrm{MeV}$ neutrons. The t'iee curves refer to the displacement models given by equations $I-6(N R T), I-7(R T-b)$, and $I-8(R T-3)$. 
In Table 6 has been plotted, relative to the $14 \mathrm{MeV}$ datum point. Included on Figure 23 are the relative values of the three models: NRT, RT-b, and RT-3. The values of the theoretical curves have been adjusted so that the "best fit" for each model was obtained. It can be seen that agreemert with the NRT or RT-b formalism is not unreasonable, but the $K T-3$ energy dependence does not describe tine data trend at all. It should be pointed out again that the extension of $\mathrm{RT}-\mathrm{b}$ and $\mathrm{RT}-3$ models to these high energies may be incorrect beyond the energy range used in the computer simulations, but useful.

\section{E. Gold Data}

A less extensive study was conducted on the gold sample. Originally, it was hoped by examintng a gold sample in the same manner as was done for the copper sample, some information on the validity of the atomic number and mass dependence of LSS theory would be obtained. However, this study could not be completed due to the low pinning rates in gold by high energy neutrons. At the flux levels available at Pajarito Site or the Van de Graaff, no change in the pinning rate was observed at the onset of irradiation. The only available source with sufficient neutron intensity produced essentially monoenergetic neutrons at $14.3 \mathrm{MeV}$. Thus the possibility of energy or flux dependence studies was eliminated. Table 7 lists the electron and neutron experiments performed 


\section{TABLE 7}

ELECTRON AND NEUTRON IRRADIATIONS ON GOLD

$\begin{array}{llllll}\text { Run No} & \begin{array}{c}\text { Irradiation } \\ \text { Energy } \\ (\mathrm{MeV})\end{array} & \begin{array}{c}\text { Temperature } \\ (\mathrm{K})\end{array} & \begin{array}{c}\text { Flux } \\ \left(\mathrm{cm}^{-2} \mathrm{~s}^{-1}\right)\end{array} & \begin{array}{c}\text { Irradiation } \\ \text { Time } \\ (\mathrm{min})\end{array} & \frac{\mathrm{dN}_{\mathrm{dy}} / \mathrm{dt}}{(\mathrm{s}-1)} \\ 13 & 2.0 \mathrm{e}^{-} & 330 & 4.00 \times 10^{10} & 13 & 1.116 \pm 0.006 \times 10^{-3} \\ 14 & 2.0 \mathrm{e}^{-} & 330 & 4.03 \times 10^{10} & 20 & 1.218 \pm 0.003 \times 10^{-3} \\ 15 & 2.0 \mathrm{e}^{-} & 330 & 1.19 \times 10^{10} & 22 & 3.93 \pm 0.01 \times 10^{-4} \\ M-B & 14.3 \mathrm{n} & 330 & 1.7 \pm 0.3 \times 10^{9} & 40 & 4.5 \pm 0.2 \times 10^{-5} \\ M-D & 14.3 \mathrm{n} & 330 & 1.8 \pm 0.4 \times 10^{9} & 31 & 4.2 \pm 0.2 \times 10^{-5}\end{array}$


on the gold sample held at $330 \mathrm{~K}$. In Figures 24 and 25 , the raw and reduced dita for a $2.0 \mathrm{MeV}$ electron irradiation (run 14) is shown. The apparent increase in the decrement at the beginning of the Irradiation was only seen at this flux level and not in the lower flux experiment. Two possibilities arise: one is that the Initial part of the 1rradlation caused the sample to heat up momentarily, while the other is the possibility of some interference with the electronfcs when the electron accelerator was turned on. Figures 26 and 27 show the raw and reduced data for run $M-D$ with $14.3 \mathrm{MeV}$ neutrons. There was no observed increase in the decrement in this run. A notable difference between the copper and gold samples was the pre-irradiation pinning. In copper this was a small effect compared to the irradiation induced pinning. In gold, during electron irradiations, the pre-irradiation pinning also appeared small (see Figure 25). For neutron irradiations with pinning rates an order of magnitude less than electron irradiations, the pre-irradiation pinning was very significant and had to be carefully assessed. This effect was presumably due to small concentrations of vacancies quenched-in during cooling. The initial pinning due to radiation induced defects was still calculated by the change in slope at the initiation of irradiation. 


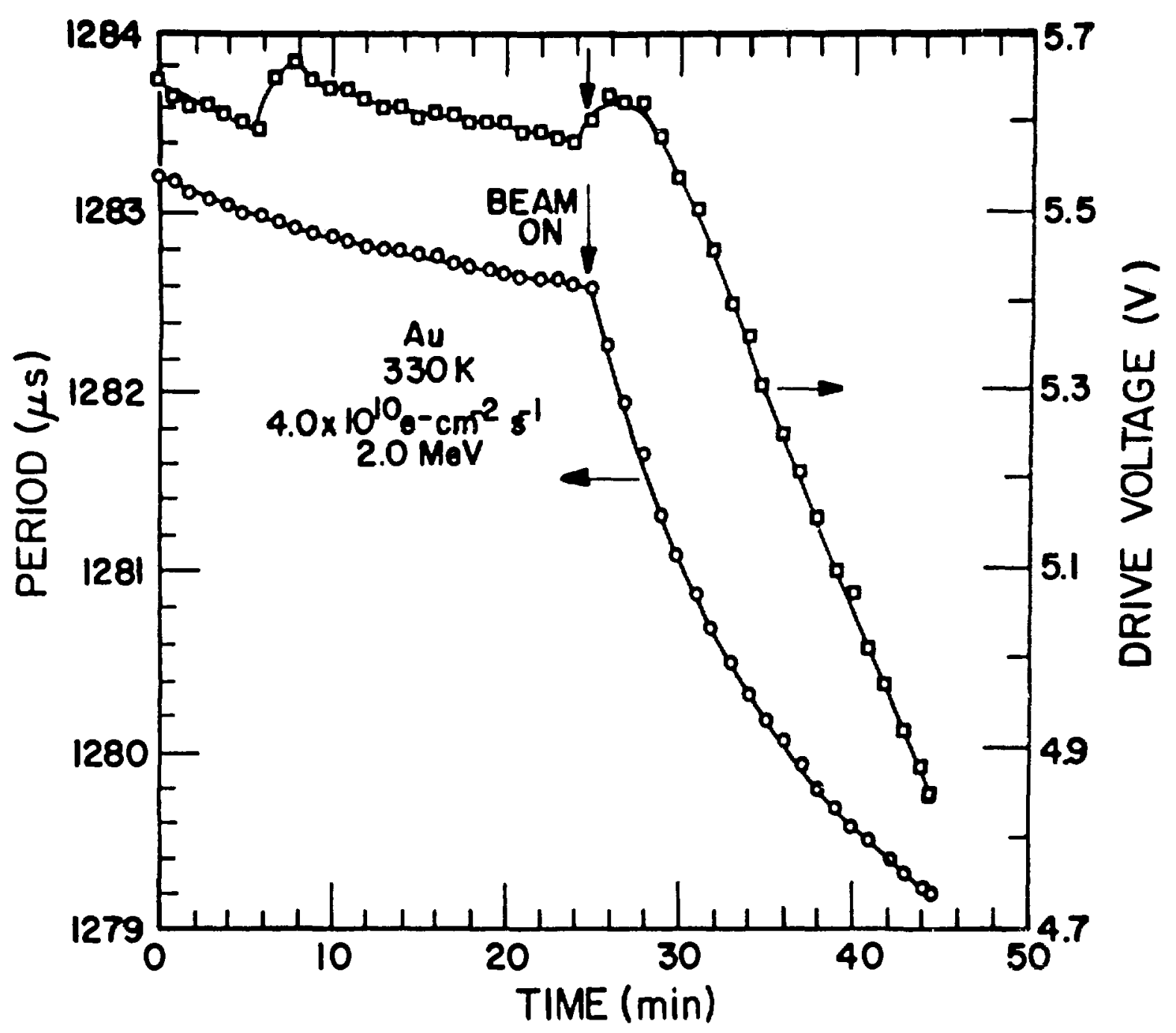

Figure 24: The raw data versus time for a $2.0 \mathrm{MeV}$ electron irradiation of gold held at $330 \mathrm{~K}$. The irradiation was begun at the beam-on pointer. 


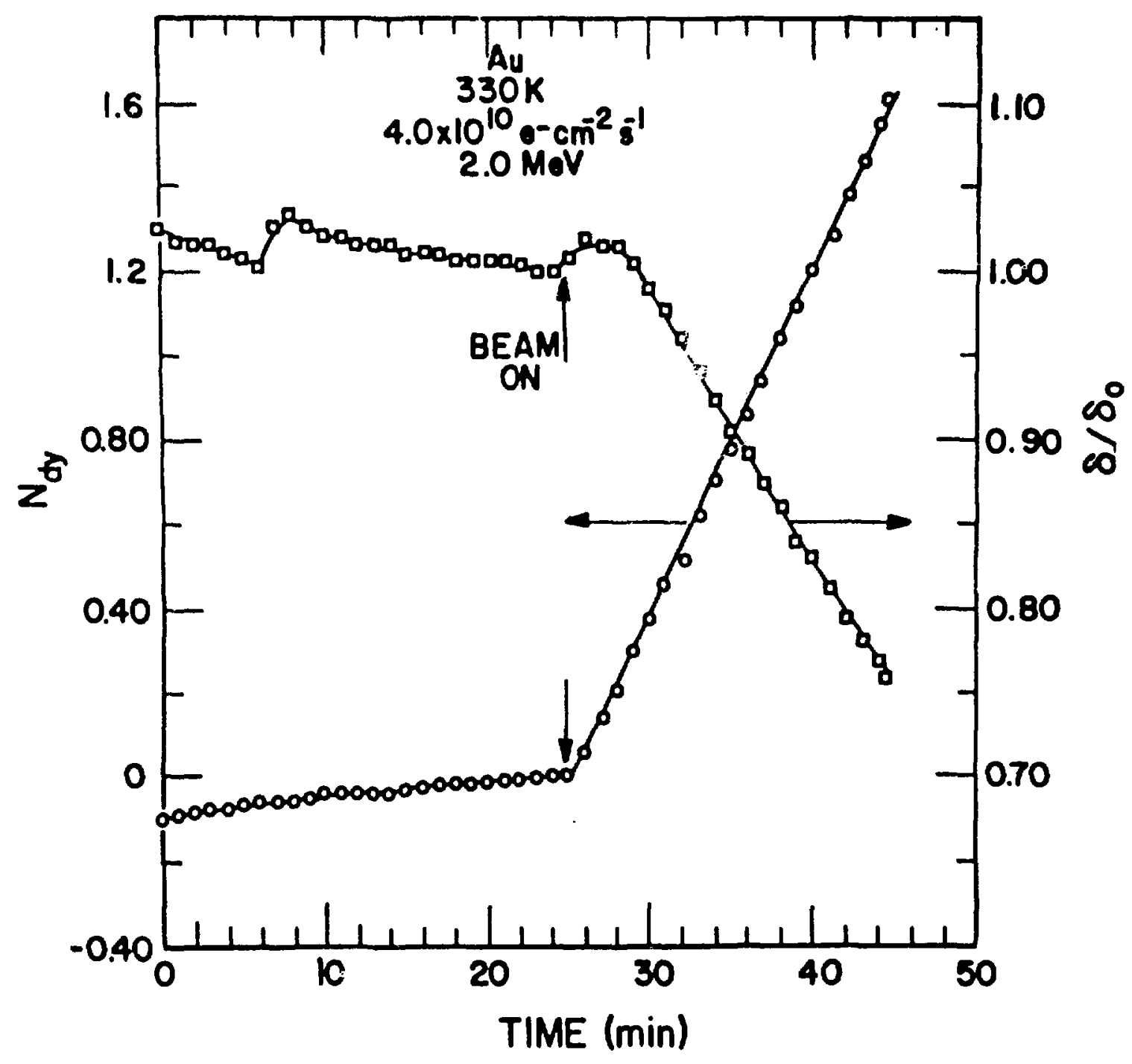

Figure 25: The reduced data from the points recorded in Figure 24 by using equations II-33 and II-43. The increase in the decrement when the beam comes on may be illusory. 


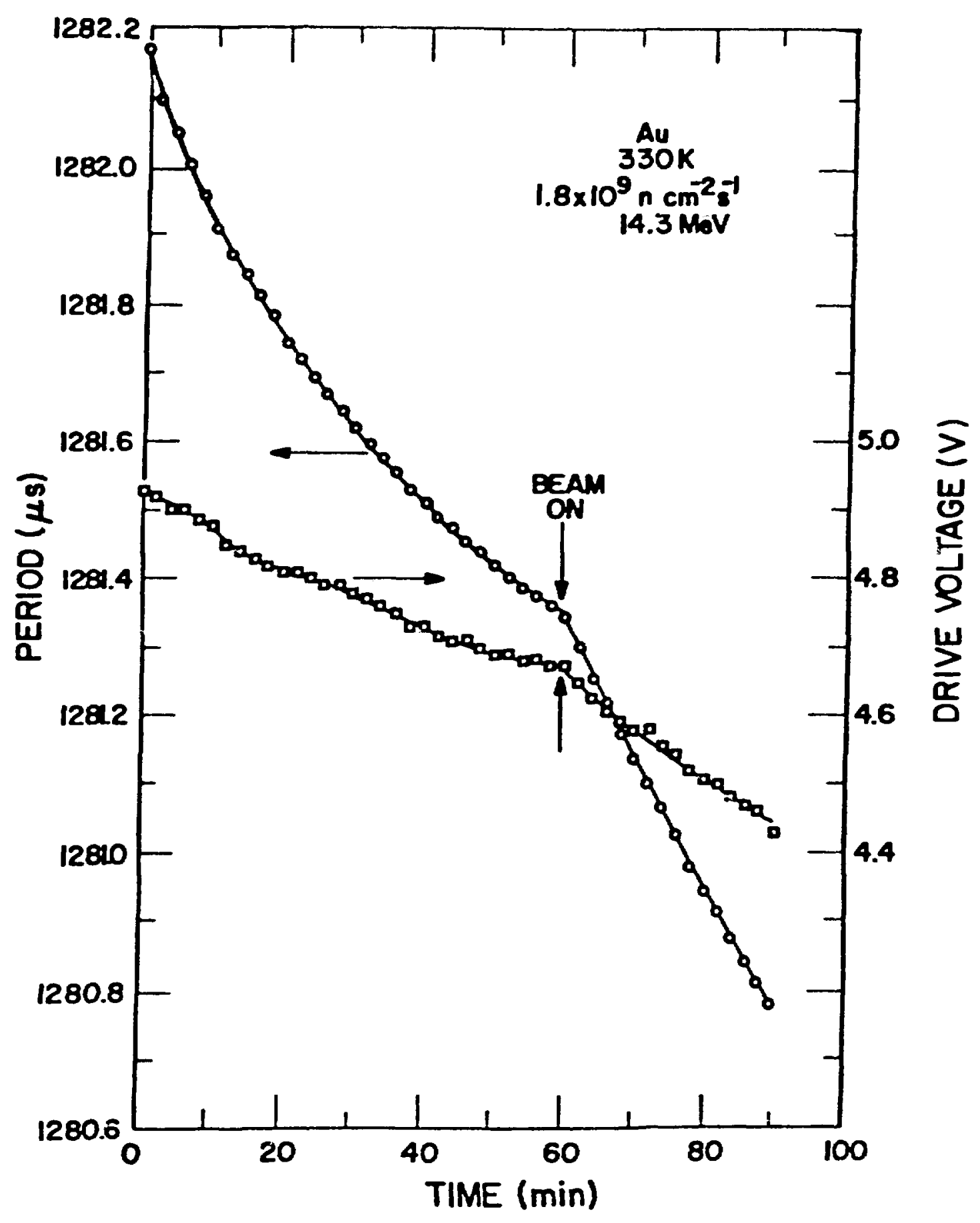

Figure 26: Raw data from run $M-D$ versus time for 14.3

MeV neutrons on gold at a flux of $1.8 \times 10^{9} \mathrm{~cm}^{-2} \mathrm{~s}^{-1}$. 


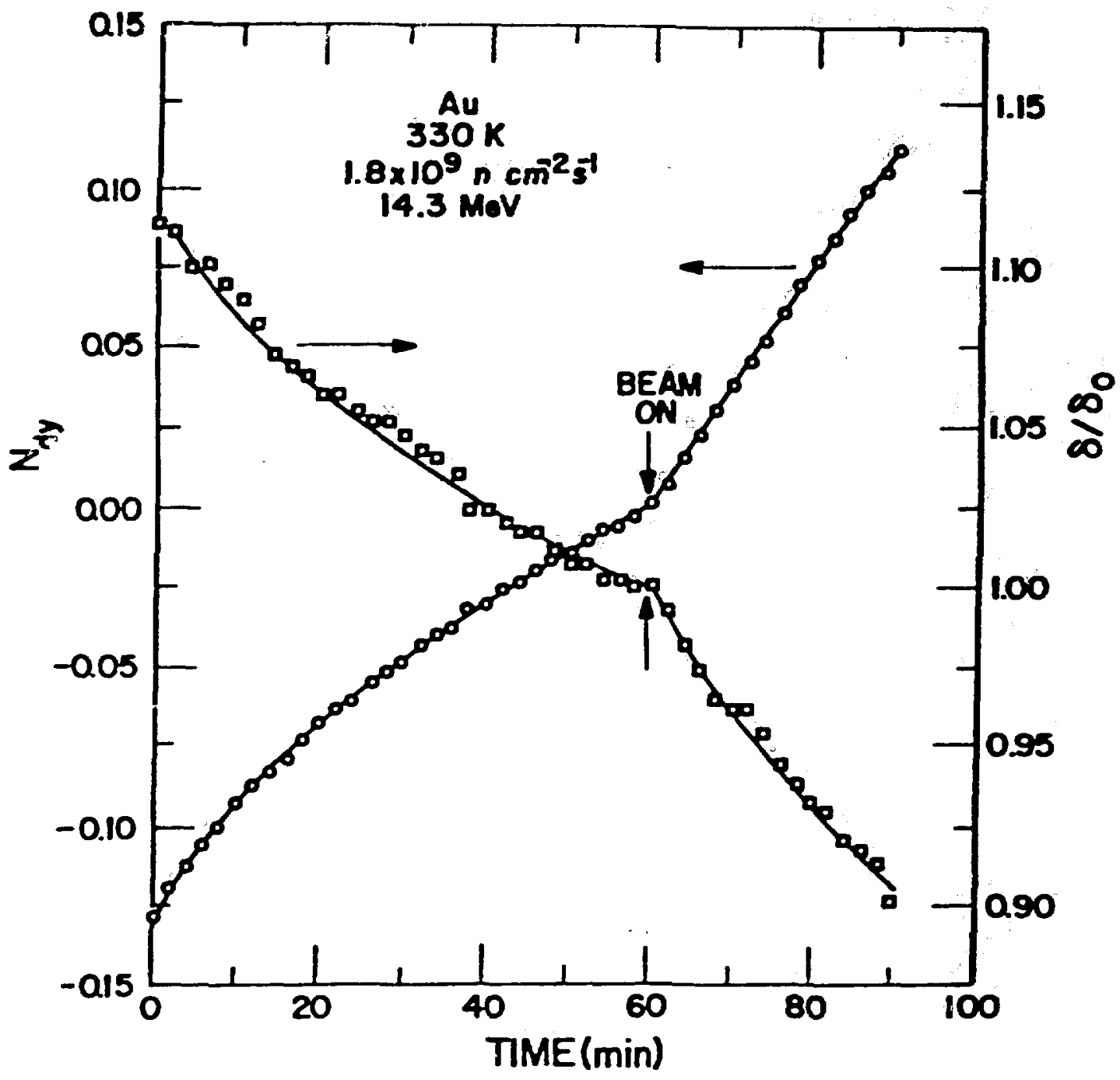

Figure 27: Reduced data from the points recorded in

Figure 26. Note that no increase in the decrement is observed. 


\section{Discussion}

\section{A. Flux Dependence In Copper}

The flux dependence of the Initial pinning rate as bhow In Figure 16 ylelds two 1mportant facts: (1) the same power law applies to electrons and neutrons and (2) the dependence 16 nong Iinear. The imporsance of the first discovery is basic to the entire experiment. In electron irradiations, interstitials produced individually and either recombine with theit associlated or another vacancy or escape annihilation to be free. Then the free interstitial undergoes random diffusion, possibly stressassisted, through the lattice to dislocations and other sinks (e.g., grain boundaries). In a neutron cascade, the local density of defects may be very high" (Si67) and interstitial interactions with depleted zones will occur. However, those interstitials" which escape the cascade and diffuse in the lattice behave in the same manner as electron-produced interstitials. Thus once the interstitial is classified as a free interstitial it loses all identity associated with the method of production. The implications of this conclusion are quite important. First, there should be no energy (neutron or electron) dependence of the flux dependence power law. Thus this data can be used to scale any other pinning rate data at a temperature of $330 \mathrm{k}$. Secondly, a direct comparison of the number of free interstitials produced during electron and neutron irradiations can be made. Since the 
free interstitial diffuses simflarly in both cases the fraction of these which reach dislocations will be identical (1.e., depleted zones do not constitute a significant competing sink).

The non-linear dependence of the initial pinning rate versus flux over three orders of magritude has not been pullished before. Thompson et al. (Th67) using gamma irradiation also reportel a non̈-linear dependence of the number of pinners on dislocations but gave no" functional relation. Brown and Simpson (Br77) using electron irradiation reported a flux dependence to the 0.85 power (no error given) at $330 \mathrm{~K}$ in good agreement with the value of $0.87 \pm 0.02$ reported here. Thus the exponent is not sample dependent. For high dose or high flux experiments on resistivity samples, another non-linear dependence was explained by a concentration shift of competing sinks, usually taken to be excess vacancies, in the lattice. For our irradiations, however, this explanation does not apply due to the low flux levels used. In the section on defect production, the number of free Frenkel pairs produced for a flux of $10^{10}$ electrons $\mathrm{cm}^{-2} \mathrm{~s}^{-1}$ is given as $1.3 \times 10^{9} \mathrm{~cm}^{-3} \mathrm{~s}^{-1}$. For copper, this is a concentration of . $1.5 \times 10^{-14}$ af $\mathrm{s}^{-1}$. For a typical irradiation time of 30 minutes (1800 seconds), the total defect concentration in the lattice, assuming no annealing, is $2.7 \times 10^{-11}$ af. For a dislocation density of $10^{9} \mathrm{~cm}^{-2}$, the atomic fraction of dislocated sites is approximately $10^{-6}$ af or five orders of magnitude larger than the 
vacancy cuncentration. Therefore, the vacancles do not offer a competing sink for interstitials during 1rradiation. Thus there appears to be no reason to refect the kinetlc equations developed In section III-B. Assuming that these equations are correct, the only conclusion left is that our understanding of dislocations is Incomplete. By fitting the data of $\mathrm{N}_{\mathrm{d} y}$ versus time (or fluence) to a quadratic polynominal, the assumption that pinning polnts are placed randomly on the dislocations must be made. Then by using the quadratic fit in time, the pinning rate at the onset of irradiation is linearly proportional to the flux. It is possible that plnning points are not randomly distributed, but it is more likely that the dislocation models which relate dislocation line length or defect drag coefficients to the modulus defect do not adequately represent the physical reality at frequencies around $1 \mathrm{kHz}$. The importance of this study lies both in the experimental value obtained for the flux dependence and in the fact that present models do not produce a correct description of this dependence.

B. Temperature Dependence in Copper

The temperature dependence of the pinning rate gives the difference between the lattice migration enthalpy and the diffusion enthalpy along a dislocation. This value is found to be $0.17 \pm 0.02 \mathrm{eV}$ from 310 to $390 \mathrm{~K}$ in agreement with previous studies. Simpson et al.(Si73, Si72a) also reported values of 
$\mathrm{H}_{\mathrm{m}}-\mathrm{H}_{\mathrm{d}}$ of $0.17 \mathrm{eV}$ for both pulsed and continuous 1rradiations. These experiments cannot, unfortunately, separate the two quant1tles $\mathrm{H}_{\mathrm{d}}$ and $\mathrm{H}_{\mathrm{m}}$. However, there exists an inftial transient or delay in the recording any detectable change in the period of vibration (see figure 20). This delay is related to the value of $H_{m}$, 1.e., to the time it takes to put sufficient numbers of pinners on the dislocation lines such that the sensitivity level of the measurement is obtalned. The steady state pinning rate then gives a value for $\mathrm{H}_{\mathrm{d}}$. Thompson et al. (Th67a) using gamma irradiation conducted an experiment with a much lower pinning rate and measured the lattjce migration enthalpy to be $0.64 \mathrm{eV}$, and the dislocation migration enthalpy to range from 0.36 to $0.51 \mathrm{eV}$. Using the average $\mathrm{H}_{\mathrm{d}}=0.44 \mathrm{eV}$, the difference $\mathrm{H}_{\mathrm{m}}-\mathrm{H}_{\mathrm{d}}=0.20$ is in reasonable agreement with our measured value of $0.17 \mathrm{ev}$.

The assumption used in calculating $\mathrm{H}_{\mathrm{m}}-\mathrm{H}_{\mathrm{d}}$ is that the flux dependence of the pinning rate is temperature independent. Thus, for experimental runs when the flux is not exactly the same as the majority of the data runs, the pinning rate is scaled to the most widely used flux value. No comprehensive study of flux dependence over this temperature range has been performed. A study was conducted by Brown and Simpson ( $\mathrm{E}-77$ ) at 330 and $573 \mathrm{k}$. As stated earlier, the exponent in the power law at $330 \mathrm{k}$ was found to be 0.85 , but the exponent at $573 k$ was 0.93 . The variation between these two extremes is not known. If one 
assumes a linear dependence on Lemperature or alternatively an exponential dependence on inverse temperature of the exponent, the value at $390 \mathrm{~K}$ would st11l be whtin tinc incertainty in the present experiment of the exponent at $330 \mathrm{~K}$. Thus at most $1 \mathrm{t}$ would introduce a $2 \%$ error in the scaled value at $390 \mathrm{~K}$. Th1s change is not likely to affect the actual value of $\mathrm{H}_{\mathrm{m}}-\mathrm{H}_{\mathrm{d}}$ but may increase the uncertainty somewhat. In conjunction with the flux deperdence measurements and the observation of the peaking effect, the discovery of the same value for the slope of the initial pinning rate versus temperature for both electrons and neutrons confirms that the same defect is responsible for dislocation pinning in both types of irradiation.

C. Free Interstitial Production Cross Secticn

Comparisons of electron and neutron irradiations yield estimates of the free interstitial production cross section. The initial slope of the curve of $\mathrm{N} d y$ versus time is preportional to the number of defects that are produced and available to pin dislocations:

$$
\frac{d N_{d y}}{d t}=\sigma_{d} \phi f p
$$

where $o_{d}$ is the displacement cross section, $\dot{\phi}$ is the flux, $f$ is the survival fraction ( 0.25 for electrons $(\mathrm{Sc} 70))$, and $\mathrm{p}$ is the fraction of free interstitials which pin dislocations. As discussed earlier, for electrons, $\sigma_{d}$ can be obtained from the electron 
energy dependence of the change in residual resistivity. From plots of $\Delta \rho / \Delta \phi$ versus electron energy, and using equation I-13, of 1 s given by:

$$
\frac{\Delta \rho}{\Delta \phi}=\sigma_{d} \rho_{F}
$$

and $\rho_{F}$ is assumed to be $1.7 \times 10^{-4} \Omega-c m$ for copper (Ju73) and $2.2 \times 10^{-4} \Omega-\cos$ for gold (Lu75). For $0.5 \mathrm{MeV}$ electrons, $\sigma_{d}=5.9$ barns for copper and for $2.0 \mathrm{MeV}$ electrons, $\sigma_{d}=42$ barns for sold. The flux in equation IV-51 is that electron flux which reproduces the same pinning rate as a given neutron flux. For convenience, the same pinning rate $\left(2 \times 10^{-5} \mathrm{~s}^{-1}\right)$ as used in computing Table 6 is chosen.

As shown in the previous two sections, the flux and temperature dependences of electron and neutron irradiations indicate that once an interstitial begins random difiusion it loses any memory of the radiation which produced it. Thus it is expected that the fraction $p$ is equal for electrons and neutrons. Now, given the same $\mathrm{dN} d \mathrm{~d} / \mathrm{dt}$ for electrons and neutrons, we have:

$$
\sigma_{n} f_{n} p_{n}^{\phi}=\sigma_{e} e^{p} e^{\phi} e
$$

where the subscripts $n$ and e refer to neutrons and electrons, respectively, and the subscript d on the displacement cross section has been dropped. Now:

$$
\sigma_{n} F_{n}=\sigma_{e} e^{\phi} \epsilon^{/ \phi} n
$$

where it has been assumed that $p_{e}=p_{n}$. Iur example, comparing 
$14.1 \mathrm{MeV}$ neutrons and $0.5 \mathrm{MeV}$ electrons, the rat $10 \phi_{\mathrm{e}} / \phi_{\mathrm{n}}$ is $21.7 \pm 0.7$ from the scaling of the flux dependence data (see. section $(11-B)$. Thus:

$$
\begin{aligned}
\sigma_{n} f & =\left(5.9 \times 10^{-24} \mathrm{~cm}^{2}\right)(0.25)(21.7 \pm 0.7) \\
& =3.2 \pm 0.3 \times 10^{-23} \mathrm{~cm}^{2} \\
& =32 \pm 3 \text { barns }
\end{aligned}
$$

assuming roughly a $10 \%$ error in the displacement cross section for electrons. By using the calculated NRT cross section of 4402 barns, then:

$$
\begin{aligned}
f_{n} & =32 \pm 3 b / 4402 b \\
& =.007 \pm .001
\end{aligned}
$$

or less than $1 \%$ of the number of defects predicted by the NRT model survive as free interstitials. Table 8 gives a list of neut ron energy, $f_{n} c_{n}$, $\sigma_{d p a}(N R T)$, and $f_{n}$ for the irradiations done in this experiment. A sligint decrease in $f_{n}$ occurs from $2.0 \mathrm{MeV}$ to $23.5 \mathrm{MeV}$ in copper.

Since no flux dependence data was taken on the gold sample, we can assume either that the pinning rate is linear with flux or that the copper flux dependence is also valid for the gold sample. We also rely on the evidence (from this and previous (Si76) studies on copper) that free interstitials are responsible for pinning in both electron and neutron irradiations of gold. By calculating the electron flux to result in the same pinning 
TABLE 8

FREE INTERSTITIAL PRODUCTION CROSS SECTIONS

\begin{tabular}{|c|c|c|c|c|}
\hline Matertal & $\begin{array}{c}\text { Neutron } \\
\text { Energy } \\
(\mathrm{MeV}) \\
\end{array}$ & $\begin{array}{l}\mathrm{fo}_{\mathrm{dpa}}(\mathrm{exp}) \\
\text { (barns) } \\
\end{array}$ & $\begin{array}{l}O_{\mathrm{dpa}}(\mathrm{NRT}) \\
\text { (barns) }\end{array}$ & E (neutron) \\
\hline $\mathrm{Cu}$ & $1.9 \pm 0.1$ & $13 \pm 4$ & 1274 & $0.010 \pm 0.003$ \\
\hline $\mathrm{Cu}$ & $3.9 \pm 0.1$ & $17 \pm 6$ & 2147 & $0.008 \pm 0.003$ \\
\hline $\mathrm{Cu}$ & $5.9 \pm 0.1$ & $20 \pm 7$ & 2792 & $0.007 \pm 0.003$ \\
\hline $\mathrm{Cu}$ & $14.1 \pm 0.1$ & $32: 3$ & 4402 & $0.007 \pm 0.001$ \\
\hline $\mathrm{Cu}$ & $23.4 \pm 0.1$ & $38 \pm 13$ & 6051 & $0.006 \pm 0.002$ \\
\hline $\mathrm{Au}$ & $14.3 \pm 1$ & $.2^{1}$ & 2273 & $0.002 \pm 0.001$ \\
\hline $\mathrm{Au}$ & $14.3: 1$ & $8 \pm 2^{2}$ & 2273 & $0.004 \pm 0.001$ \\
\hline \multicolumn{5}{|c|}{${ }^{1}$ Calculated using the 0.87 power law } \\
\hline
\end{tabular}


rate as the neutron flux of $1.8 \times 10^{9} \mathrm{~cm}^{-2} \mathrm{~s}^{-1}$, we get

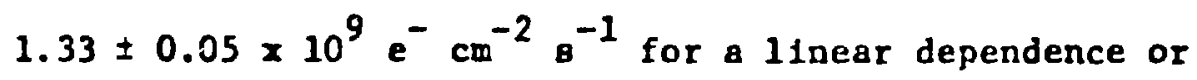
$8.8 \pm 0.4 \times 10^{8} \mathrm{e}^{-2} \mathrm{~cm}^{-2} \mathrm{~s}^{-1}$ using the 0.87 power 1aw. These values give an experimental free interstitial production cross section of $8 \pm 2$ barns or $5 \pm 2$ barns, respectively. When compared with the NRT cross section of 2300 barns, the survival fraction of free interstitials in gold is $0.004 \pm 0.001$ or $0.002 \pm 0.001$ for linear or $\phi^{.87}$ flux dependencies, respectively. These values are sumnarized in Table 8 . Agaln only a small fraction (< $1 \%$ ) of the NRT defect concentration survives as free interstitials. The number of free interstitials surviving at room temperature is a subset of the number of interstitials surviving athermal recombination, as discussed in section $C$ of the introduction. Add1tionally, the number of interstitials which annihilate in stage $I$ is also proportional to the number surviving athermal recombination. Thus the absence of stage I annealing in fast neutron irradiated gold (Ho73) indicates that only a small fraction of the produced interstitials survive athermal recombination. Therefore, it is expected that only a small fraction of the total number of izterstitials produced remain as free interstitials at room temperature. This is in agreement with our experimental observation of less than $0.5 \%$ survival. 
From experimental values of the free interstitial

production cross sections, the damage energy cross sections, and the total neutron scattering cross sections, an estimate of the number of free defects per primary knock-on atom (PRA) or per unit damage energy can be made. In Table 9, the number of free defects is listed for copper and gold versus the energy of the Incoming neutrons. Colums 6 and 7 of Table 9 are calculated from the following equations:

$$
\begin{aligned}
& \text { colurm 6: } \quad f \sigma_{\mathrm{dpa}} / \sigma_{\mathrm{T}} \\
& \text { columm 7: } \mathrm{f} \sigma_{\mathrm{dpa}} / \sigma_{\mathrm{T}} \overline{\mathrm{T}}_{\mathrm{dam}}
\end{aligned}
$$

where $\overline{\mathrm{T}}_{\mathrm{dam}}=\sigma_{\mathrm{DE}} / \sigma_{\mathrm{T}}$ is the average damage ener $\mathrm{gy}$ for the recoil spectrum. As seen in Table 9, the number of free interstitials per cascade or PKA increases with increasing neutron energy, but the number per unit damage energy decreases. lising the ofphs of Sigmund et al. (Si71a), the range of the damage increases from roughly $250 \AA$ at $2.0 \mathrm{MeV}$ to $1100 \AA$ at 20 MeV. Thus while the smaller cascade at $2 \mathrm{MeV}$ produces fewer interstitials, the smaller number per unit damage energy of the $20 \mathrm{MeV}$ cascade indicates the higher density of defects. Thus the damage effectiveness of the higher energy neutrons is less than that of lower energy neutrons.

The validity of the comparison of the experimental production cross sections to the calculated total defect production 
TABLE 9

FREE INTERSTITIALS PER CASCADE

\begin{tabular}{|c|c|c|c|c|c|c|}
\hline Material & $\begin{array}{c}\text { Neutron } \\
\text { Energy } \\
(\mathrm{MeV}) \\
\end{array}$ & $\begin{array}{r}\sigma(D E) \\
(b-k e V) \\
\end{array}$ & $\begin{array}{c}\sigma_{T} \\
\text { (barns) }\end{array}$ & $\mathrm{fo}_{\mathrm{dpg}}(\mathrm{b})$ & $\begin{array}{l}\text { Number of } \\
\text { free Inter- } \\
\text { 8t1tials/ } \\
\text { PKA } \\
\end{array}$ & $\begin{array}{c}\text { Number of free } \\
\text { Interst1t ala/ } \\
T_{\text {dam }} \\
\left(\mathrm{keV}^{-1}\right) \\
\end{array}$ \\
\hline $\mathrm{Cu}$ & $1.9 \pm 0.1$ & 82.7 & 3.04 & $13 \pm 4$ & $4 \pm 1$ & $0.16 \pm 0.05$ \\
\hline $\mathrm{Cu}$ & $3.9 \pm 0.1$ & 137 & 3.61 & $17 \pm 6$ & $5 \pm 2$ & $0.12 \pm 0.04$ \\
\hline $\mathrm{Cu}$ & $5.9 \pm 0.1$ & 177 & 3.83 & $20 \pm 7$ & $5 \pm 2$ & $0.11 \pm 0.04$ \\
\hline $\mathrm{Cu}$ & $14.1 \pm 0.1$ & 275 & 2.95 & $32 \pm 3$ & $11 \pm 1$ & $0.11 \pm 0.04$ \\
\hline $\mathrm{Cu}$ & $23.4 \pm 0.1$ & 379 & 2.51 & $38 \pm 13$ & $15 \pm 5$ & $0.10 \pm 0.04$ \\
\hline Au & $14.3 \pm 0.1$ & 227 & 5.70 & $5 \pm 2$ & $0.9 \pm 0.3$ & $0.02 \pm 0.01$ \\
\hline Au & $14.3 \pm 0.1$ & 227 & 5.70 & $8 \pm 2$ & $1.4 \pm 0.3$ & $0.04 \pm 0.01$ \\
\hline
\end{tabular}


cross fiection to obtain $f_{n}$ is based on the nature of the displecement cascades at high recoll energies. Robinson (Ro71) presented a qualitative discussion of the change in defect density In a displacement cascade as a function of increasing primary recoll energy. In the regime where electronic energy losses cannot be ignored, the fraction of total defects surviving in the cascade w11l be nearly constant with increasing primary recoll energy. Additionally, the defect density will be independent of the recoll energy. Thus the fraction of free Interstitials will also be nearly constant for these recoll energies. An estimate of the recoll energy at which this leveling off process occurs can be obtained frow the computer simulations of Doran et al. (Do75) which estimate the number of residual mobile vacancies as a function of recoil energy (see equation 1-9). For extrapolation of tinis model above recoil energies of $100 \mathrm{keV}$, the number of mobile vacancies is a linear function of the damage energy and is approximateiy a constant $6 \pi$ of the total number of defects predicted by the NRT model. This fraction increases slowly with decreasing primary recoil energy to $13 \%$ at $5 \mathrm{keV}$. Below $5 \mathrm{keV}$, a mere rapic increase in the mobile fraction of vacancies occurs, rising to $86 \%$ at $0.1 \mathrm{ker}$. Thus essentially, the large changes in cascade structure occur below $5 \mathrm{keV}$. Experimental evidence of constant defect density is the observation by Merkle (Me66) that no significant change in the size distribution of defects in 
self-ion irradiated copper occurred with Increasing irradiation energy. This indicates that the basic unit, the subcascade, of damage in copper at high recoll energies stays the same and interference among subcascades is negligible.

The comparison of the measured neutron energy dependent pinning rate (as shown in Figure 23 and Tables 8 and 9) and the NRT model supports the above discussion. For recoll spectra due to $1.9,14.1$, and $23.4 \mathrm{MeV}$ neutrons, the percentage of recolls above $5 \mathrm{keV}$ is $87.3 \%, 92.8 \%$, and $95.3 \%$, respectively. Therefore, large changes in the free interstitial survival fraction are not expected for the neutron energy range covered in this experiment. This is substantiated by the slow decrease in the survival fraction, $f_{n}$, from 2 to $24 \mathrm{MeV}$ as seen in Table 8 . Moreover, if low energy (below $5 \mathrm{keV}$ ) recoils are the dominant producers of free interstitials, the number of free interstitials per PKA would remain almost constant (possibly decrease slightly due to a smaller percentage of recoils below $5 \mathrm{keV}$ ) with increasing neutron energy. However, in Table 9 the number of free interstitials per PKA increases from 4 at $1.9 \mathrm{MeV}$ to 15 at $23.4 \mathrm{MeV}$. Thus, our experimental evidence supports the arguments in the previous paragraph and our comparison of the experimental data to the NRT model is valid. A model such as RT-3 does not agree with the discussion in the previous paragraph. If comparison of experimental free interstitial production cross sections with calculated 
RT-3 displacement cross sections is made, the survival fraction of interstitials increases from 0.016 at $5.9 \mathrm{MeV}$ to 0.030 at 23.4 MeV.

From the number of free interstitials produced, the number of surviving vacancies can be estimated. Various TEM results of defect type distributions were discussed in the introduction. The problem with using TEM results to estimate relative interstitial and vacancy clustering is the uncertainty in Identifying the type of defect cluster. Merkle (Me66) reported essentially no observation of interstitial clusters after fast neutron irradiations of copper and gold. Rühle (Ru70), on the other hand, reported that the concentration of interstitials in clusters was 0.8 times the concentration of vacancies in clusters. These irradiations were done with a spectrum of neutron energies and low energy neutrons may contribute considerable numbers of free defects. Beeler (Be66), through computer simulations, indicated that only $10 \%$ of the interstitials which escape recombination are associated with another defect. Using Merkle's and Beeler's results, the estimated total number of vacancies which do not recombine with an interstitial is 1.1 times the number of free interstitials measured in this experiment. This number includes free and clustered vacancies.

For gold using $14 \mathrm{MeV}$ neutrons, Merkle (Me74) reported only vacancy clusters in gold. Additionally, Merkle computed the 
cascade-cluster efficlency to be roughly $30 \%$ of the NRT value. Thus, we see that our supposition of no interstitial clustering 18 not consistent with TEM defect concentrations. Several sources of error may be proposed. The ability to resolve the nature of black-white contrast features in the TEM depends heavily on the abllity to measure depth in the sample. It is possible that the Identity of the defect clusters has been misinterpretated. Altemately, the problem may IIE in the use of thin folls for TEM irradiation samples. The interaction of interstitials with the surface may deplete their numbers while enhancing the vacancy concentration. Another TEM 11mitation may be the inability to resolve the interstitial clusters at low doses due to their small size. In addition, it may be that interstitials are in clusters large enough to be resolved, but the concentration of clusters is so small that the statistics are poor. All in all, the problem seems to lie with the inability to determine the magnitude of interstitial clustering.

A rough order of magnitude comparison with void experiments indicates whether or not our estimate of vacancy production is at all reasonable. The number of vacancies contained in voids will come from both free vacancies and the emission of vacancies by depleted zones. Our experiment measures the number of free interstitials at the end of short-term-annealing (STA) of a displacement cascade. From this number we estimated the number 
of vacancies at the end of STA to be 1.1 times the free

interstitial concentration. Vold growth occurs over a much longer span of time than STA, but the vacancies avallable for vold growth are those present at the end of STA. Obviously, some vacancles will rema1n in other sinks, so the concentration in volds is a lower $11 \mathrm{mit}$ on the number produced. Brimhall and Mastel (Br69) performed neutron 1rradiations on copper at $533 \mathrm{~K}$ $\left(0.39 \mathrm{~T}_{\mathrm{m}}\right)$ and found for a dose of $1.2 \times 10^{20}$ neutrons $\mathrm{cm}^{-2}$ $(E>1 \mathrm{MeV})$, a vold density, $\mathrm{N}_{\mathrm{v}}$, of $3.4 \times 10^{14} \mathrm{~cm}^{-3}$ and an average size of $230 \AA$. This gives a total vacancy number stored in voids (assumed to be octahedral) of $2 \times 10^{20}$. At this point we ignore the difference in short-term annealing due to the temperature at $533 \mathrm{~K}$ as opposed to $330 \mathrm{~K}$ used in this experiment. Using the number of free interstitials per cascade at $2 \mathrm{MeV}$ as a lower estimate on the total number of vacancies created, at least $4.8 \times 10^{20}$ vacancies are produced by the given dose. So less than half the predicted vacancy concentration is stored in voids. The fact that the predicted number of vacancies is larger than, and the same order of magnitude as, the number of vacancies in voids gives some credibility to the number of vacancies predicted by this experiment.

Another experiment which attempted to measure the number of free defects in a fission neutron irradiation environment was conducted by Blewitt et al.(B171). A comparison of the amount of 
order Induced during Irradiation of a disordered $\mathrm{Cu}_{3} \mathrm{Au}$ sample by thermal and f1saton neutrons was done using electrical resistivity measurements. These experiments were done at $423 \mathrm{~K}$ with an average fission neutron energy of $0.65 \mathrm{MeV}$, giving an average damage energy of $25 \mathrm{keV}$ and a displacement number of 400 . By assuming that the number of free vacancies produced in the thermal neutron case was known, the number liberated in the fission irradiation could be established. This number was estimated to be 3 to 7 vacancies per cascade, or less than 27 of the NRT value. This is a good agreement with the present experiment wilch yields a 17 escape number for interstitials at $2 \mathrm{MeV}$. For lower energy neutrons this percentage shoula rise as shown by the trend in Table 8. Both experiments show that a small fraction of defects escape the cascade and freely migrate.

\section{Comparison to Computer Models}

In Figure 23, the number of pinning points per neutren at constant damage rate is plotted against neutron energy. The three curves represent the various models of defect production as a function of neutron energ:. The $\operatorname{Ni} T$ and $R T-b$ models reasonably represent the trenc of the cate, winjle the F.I-3 mocel does not agree with the data trend at ali. Uncertainties in the mode? calculations are due to the limited validity of the models(computer simulations only cover the range of $I_{\text {dam }}$ to a fes tens of keV'), 
the poorly defined parameters, and the accuracy of tabulated cross sectlons. One problem basic to all computer simulations of damage 15 the use of the LSS electronlc energy loss theory (see equation I-5). The basic assumptions of the LSS theory break down at higher energies, and the value of $\mathrm{T}_{\mathrm{dam}}$ wjil tend to be an overestimate. Robinson (Ro75) noted that ISS theory tends to lose 1ts validity at energies greater than $(0.27 \mathrm{keV}) \mathrm{Az}^{1.4}$. For copper and gold, the values of this energy are $180 \mathrm{keV}$ and $2.1 \mathrm{MeV}$, respectively. Thus for copper, at neutron energies above a few MeV, the three models may actually overpredict the displacement cross section. As seen in Table 1, 77.5\% of the damage energy at $14 \mathrm{MeV} 1 \mathrm{~s}$ attributable to recoils above $200 \mathrm{keV}$, so errors in displacement cross section may be significant. The problem of parameter definition lies in the choice of $E_{d}$ or the recombination radius, as varying these quantities can alter the density of the cascade and thereby influence the number of defects surviving recombination. Since neutron cross sections are not particularly well-known above 15 to $20 \mathrm{MeV}$, an evaporation model (Pa74) is used in which the high energy cross section is assumed to be energy independent. All of the above models generate a displacement cross section for the total number of stable defects surviving athermal recombination. However, at $330 \mathrm{~K}$ some additional annealing occurs since interstitials are reasonably mobile. The extension of computer simulations through the short-term annealing stage 
( $10^{-6}$ seconds) has been carrled out by Doran (Do70), Doran Et al. (Do75, Do76a), and Beeler (Be75, Be76). Unfortunately, these simulations suffer some of the same problems as the cascade simulations on which they rely. These models rarely extend to PKA energies above $20 \mathrm{keV}$, although a few calculations have been done at higher energles (100 keV, Do75, and $5 \mathrm{MeV}, \mathrm{Be} 75$ ). Some differences in the predictions of the number of free defects at these low PKA energies (20 keV or less) distinguish one cascade simulation model from another. The Beeler results predict dense cascades with vacancy and interstitial clustering and a small percentage of free defects. The RT-3 cascades show less interstitial clustering, but more vacancy clustering, and a larger number of free defects. Additionally, the RT-3 model predicts a significant decrease in damage efficiency at higher PKA energies. For example at a PKA energy of $20 \mathrm{keV}$ in $\gamma$-iron, the Beeler cascades predict half the number of residual defects predicted by the RT-3 cascades (Od76). Only $18 \%$ of initial defects survive the annealing of a Beeler cascade, while $65 \%$ survive the RT-3 cascade (note that different pre-anneal defect numbers are predicted by these two models). Given the relatively slow decrease in the damage efficiency as given in Table 9 and the few number of free interstitials, this experiment's results would tend to confirm a denser cascade model such as Beeler's over the more spread out cascade results of the RT-3 models. 


\section{v. Conclusions}

\section{A. Summary of Results}

The data presented here have shown that the technique of Internal friction measurements is a viable way to study the inftial damage rates in low flux neutron irradiations. This is possible because of the large response of the modulus defect to the addition of a few pinning points to the dislocation lines. The flux dependence of the initfal pinning rate in copper was shown to be a non-linear function in contrary to the predictions of simple kinetic and dislocation models. The data were fit to a power law dependence on the flux with the exponent being $0.87 \pm$ 0.02 for both neutron and electron irradiations (see Figure 16). The dependence of the pinning rate on temperature was also examined. The activation enthalpy for both neutron and electron data was found to be $0.17 \pm 0.02 \mathrm{eV}$ over the range from 310 to $390 \mathrm{~K}$ (see Figure 19). The combination of these results indicated that the same defect (self-interstitial) was responsible for dislocation pinning in both types of irradiation. Furthermore, since the interstitial was free in electron irradiations, the interstitial observed in neutron irradiations was also free and lost any jdentity which associated it with a neutron displacement cascade.

A study of the neutron energy dependence of the initial 
pinning rate in copper was also conducted. When projected to hıgh energy neutron recolls, the NRT and RT-b damage models agreed with the trend of the data better than the RT-3 model (see Figure 23). By comparison of equal pinning rates during electron and neutron irradiations, estimates of the free interstitial production cross section were made (see Table 8). Th1s cross section was found to be $1 \%$ or less of the total displacement cross section of the NRT model. Moreover, the number of interstitials escaping a cascade was calculated (see Table 9) to increase with increasing neutron energy; but the damage effectiveness of the higher energy neutrons was less than that of the lower energy neutrons (see Table 9). The total number of vacancies produced by a cascade was estimated, by assuming little interstitial clustering, to be 1.1 times the number of free interstitials. The small fraction of residual defects was found to be in disagree ent with short-term annealing simulations of Doran which predict $6 \%$ of the NRT number of free vacancies. Furthermore, TEM results indicated that a higher fraction of defects were left in the lattice. Thus it was suggested that TEM interpretation of the type of defect cluster was in error or numerous small interstitial clusters existed. It was also suggested that due to the small fraction of free interstitials produced, a theory which favored dense cascade structure would be more appropriate than one which predicted a less dense cascade structure. 


\section{B. Extensions of the Present Experiment}

As this technique has been shown to be rellable and applicable, the possibllity of exploring several radiation damage problems exists. Low energy neutron experiments could be carried out with $2.0 \mathrm{MeV}$ to $0.2 \mathrm{MeV}$ neutrons using a $\mathrm{ll}(p, n)$ source. These experiments could explore the applicabllity of the dafect models at lower energles. Since the maximum recoll energy in copper from a $0.2 \mathrm{MeV}$ neutron is $12 \mathrm{keV}$, thj; experiment would also be in the range of computer simulations of cascades. Obviously, the same data reduction techniques could be applied to this data and the free 1nterstitial production studied to 0.2 MeV. With this Information in hand, one could use various neutron spectra to study the integrating properties of the damage spectrum. Experiments done at facilities such as a $B e(d, n)$, fission, reactor, or LAMPF beato stop sources would study a wide range of neutron spectra. A comparison with the displacement cross section predicted by different models could then be made by integrating equation I-10 over the neutron energy spectrum. Another experiment of interest in radiation damage which could be performed by this technique would be the study of the pinning rate due to $10-20 \mathrm{MeV}$ protons. It has been suggested that $14 \mathrm{MeV}$ neutron damage, which is important in fusion energy applications could be simulated by $16 \mathrm{MeV}$ protons (Mi73, Lo73, 0m77). The PKA recoil energy spectrum of $16 \mathrm{MeV}$ protons closely 
reproduces that from $14 \mathrm{MeV}$ neutrons, hence the TEM cxperiments (M173) show good agreement in size and density of defect clusters. However, proton PKAs have a higher fraction of low energy transfer collisions than $14 \mathrm{MeV}$ neutrons, thus higher parcentages of free defects would be expected. Electrical resistivity measurements (Th7j) show a simflarity to fast neutron irradiations of Horak and Blewtt (Ho73). If the relative number of simple defects is higher for proton irradiations than neutren frradiations, the highly sensitive dislocation pinning measurement would be the best way to study this problem. Then, a conclusion on the validity of this simulation technique could be made.

Several other interesting studies could also be performed using this technique. Higher neutron fluxes could be obtained at RTNS-II (Lawrence Livermore Laboratory) or WNR(LASL) to extend the neutron flux to $10^{11} \mathrm{~cm}^{-2} \mathrm{~s}^{-1}$. This information would confirm the high end of the flux dependence measurements and approach proton flux levels to help examine simuiation possibilities. A study of the isochronal annealing properties of proton and neutron irradiated copper would yield information on the cluster break-up and diffusion properties of the free defects. Again some information on the validity of simulaticn techniques would be obtained. A more thorough examination of the flux dependence of the pinning rate could be done at lower flux levels to study the delay before an observable change in the modulus defect occurs. It is possible 
that information on the defect-dislocation interaction may be obtained. By combining internal friction, electrical resistivity, and vold density measurements the range of dose levels from $10^{10}$ to $10^{22}$ neutrons $\mathrm{cm}^{-2}$ could be spanned in the same materlal. A correlation between low dose and high dose experiments may be found. Obviously, all of the above experiments and the experiment presented in this thesis could be extended to other materials such as platinum, niobium, molybdenum, aluminum, and silver. Then the mass dependence of neutron damage can be explored. 
Acknowledgnents

I wish to acknowledge the aid given me by several individuals and organizations during the course of this work. First, I thank my two advisors, A. A. Coland of Jrookhaven National Laboratory and an adjunct profissor of the State Lniversity of New York at Stony Brook, and 1). M. Parkin of Los Alamos Sirjentific Laboratory, who sughested the topic of the thesis and guided the course of the work. Second, I thank H. Mitche]1 Simpson of Wright State University who designed and fabricated the phase-locked-loop and temperature control systems, fave valuable assistance during electron and neutron irradiations, and provided useful discussions of the data. Also, I thank R. K. Smith for his assistance in doing experiments at the LASL Van de fraaff facility. B. Bayhurst, R. Prestwood, and J. Gilmore, of the LASL Radiochemistry Group, gave much needed assistance with the neutron dosimetry and flux calculations. Finally, I thank all members of the LASI, Physical Chemistry and Metallurgy Group who provided useful discussions and advice throughout the course of this study. Financial support was furnished by Los Alamos Scientific Laboratory through W. Green of the Physical Chemistry and Metallurgy Group, and later, by a Fellowship granted by Associated Western Universities, Inc . 


\section{References}

[Al61] G. A. Alers and D. O. Thompson, J. Appl. Phys. 32, 283 (1961).

[Ba64] W. Bauer and A. Sosin, Phys. Rev. A 135, 521 (1964).

[Ba68] T. O. Baldwin, F. A. Sher111, and F. W. Young, Jr., J. Appl. Phys. 39, 1541 (1968).

[Be64] M. J. Berger and S. M. Seltzer, "Tables of Energy Losses and Ranges of Electrons and Positrons," National Aeronautics and Space Agency Report NASA SP-3012 (1964).

[Be66] J. R. Beeler, Jr., Phys. Rev. 150, 470 (1966).

[Be75] J. R. Beeler, Jr. and M. F. Beeler, Proc. Intern. Conf. Fundamental Aspects of Radiation Damage in Metals, Gatlinburg, 1975, v. I, p. 28.

[Be76] J. R. Beeler, Jr, and M. F. Beeler, Proc. Intern. Conf. Computer Simulation for Materials Applications, Gatlinburg, 1976, p. 51.

[B171] T. H. Blewitt, A. C. Klank, T. Scott, and W. Weber, Prcc. Intern. Conf. Radiation-Induced Voids in Metals, Albany, 1971 , p. 757.

[Bo67] A. Bourret and D. Dautreppe, Phys. Status Solidi 24, K173 (1967).

[Br69] J. Brimhall and B. Mastel, J. Nucl. Mater. 29, 123 (1969).

[Br77] G. J. Brown and H. M. Simpson, Wright State University, unpublished data, (1977).

[Bu64] G. Burger, H. Meissner, and $W$. Schilling, Phys. Status Solidi 4,281 (1964).

[Co62] R. R. Coltman, Jr., C. E. Klabunde, D. L. McDonald, and J. K. Redman, J. Appl. Phys. 33, 3509 (1962) .

[Co67] R. R. Coltman, C. E. Klabunde, and J. K. Redman, Phys. Rev. 156, 715 (1967).

[De73] P. H. Dederichs, J. Phys. F 3, 471 (1973).

[Do70] D. G. Doran, Radiat. Eff. 2, 249 (1970). 
[Do75] D. G. Doran, R. L. Simons, and W. M. McElroy, in Properties of Reactor Structural Alloys after Neutron or Particle Irrad1at1on (ASTM, Philadelph1a 1975), P. 290.

[Do76] D. G. Doran and N. J. Graves, Hanford Engineering Development Laboratory Report HEDL-TME 76-70, (1976).

[Do76a] D. G. Doran and J. 0. Schiffgens, Proc. of the Workshop on Correlation of Neutron and Charged Particle Damage, Oak Ridge, 1976 , p. 3.

[Ea69] J. A. Eades, Ph1los. Mag. 19, 47 (1969).

[Fu76] C. Y. Fu and F. G. Perey, J. Nucl. Mater. 61, 153 (1976).

[Ga76] T. A. Gabriel, J. D. Amburgey, and N. M. Greene, Nucl. Sc1. Eng. 61, 21 (1976).

[Gr56] A. Granato and K. Lücke, J. Appl. Phys. 27, 583, 789 (1956).

[Gr70] E. E. Gruber, J. A. Tesk, T. H. Blewitt, and R. E. Black, Phys. Rev. B 2, 2849 (1970).

[Gr74] E. E. Gruber, T. H. Blewitt, and T. O. Baldwin, J. Appl. Phys. 45, 542 (1974).

[Gu77] M. W. Guinan and C. E. Violet, Symposium on Neutron CrossSections from 10 to $40 \mathrm{MeV}$, Upton, 1977, p. 361.

[He67] C. R. Heiple and H. K. Birnbaum, J. Appl. Phys. 38, 3294 (1967).

[Ho72] J. A. Horak and T. H. Blewitt, Phys. Status Solidi A 9 , 721 (1972).

[Ho73] J. A. Horak and T. H. Blewitt, J. Nucl. Mater. 49, 161 (1973).

\{Ho75\} J. A. Horak and T. H. Blewitt, Nucl. Techn. 27, 416 (1975).

[Ip70] M. Ipohorski and L. M. Brown, Philos. Mag. 22, 931 (1970).

[Ju73] P. Jung, R. L. Chapin, H. J. Fenzl, K. Reichelt, and P. Wombaugh, Phys. Rev. B 8,553 (1973).

[Ke65] D. Keefer, J. C. Robinson, and A. Sosin, Acta Metal1. 13, 1135 (1965). 
[K155] G. H. Kinch1n and R. S. Pease, Rep. Prog. Phys. 18, no. 1 (1955).

[Ko52] J. S. Koehler, Imperfections In Nearly Perfect Crystals (J. Wiley and Sons, New York, 1952) p. 197

[La71] B. C. Larson and F. W. Young, Jr., Phys. Rev. B 4, 1709 (1971).

[La75] B. C. Larson, J. Appl. Crystallogr. 8, 150 (1975).

[La77] B. C. Larson and F. W. Young, Jr., J. Appl. Phys. 48, 880 (1977).

[L163] J. Lindhard, M. Scharff, and H. E. Schidtt, Mat. Fys. Medd. Dan. V1d. Selsk. 33, no. 14 (1963).

[Lo73] C. M. Logan, J. D. Anderson, and A. K. Mukherjee, J. Nucl. Mater. 48, 223(1973).

[Lu75] P. Lucasson, Proc. Intern. Conf. Fundamental Aspects of Radiation Damage in Metals, Gatlinburg, 1975, v.I, p. 42.

[Ma61] M. J. Makin, A. I. Whapham, and F. J. Minter, Philos. Mag. 6, 465 (1961).

[Ma62] M. J. Makin, A. D. Whapham, and F. J. Minter, Philos. Mag. ?, 285 (1962).

[Ma63] M. J. Makin and S. A. Manthorpe, Philos. Mag. 3, 1725 (1963).

[Mc48] W. A. Mckinley and H. Feshbach, Phys. Rev. 74, 1759 (1948).

[Mc67] K. G. McIntyre, Philos. Mag. 15, 205 (1967).

[Me65] K. L. Merkle, in Radiation Effects (Gordon and Breach, New York, 1965), p. 173.

[Me66] K. L. Merkle, Phys. Status Solidi 18, 173 (1966).

[Me74] K. L. Merkle, Nuc1. Techn. 22, 66 (1974).

[Me76] K. L. Merkle, in Radiation Damage in Metals (ASM, Metals Park, 1976), p. 58 .

[Mi73] J. B. Mitche11, C. M. Logan, and C. J. Echer, J. Nucl. Mater. 48, 139 (1973). 
[Mo48] P. M. Morse, Vibration and Sound (McGraw-ll11.2 Book Co., New York, 1948).

[No69] D. I. R. Norr15, Philos. Mag. 19, 527 (1969).

[No72] A. S. Nowlck and B. S. Berry, Anelastic Relaxation in Crystalline Sollds (Academic Press, New York, 1972).

[No75] M. J. Norgett, M. T. Rob1nson, and I. M. Torrens, Nuc1. Eng. Des. 33, 50 (1975).

[0d76] G. R. Odette and D. R. Dorion, Nucl. Techn, 29, 346 (1976).

[Oen73] 0. S. Oen, "Cross Sections for Atomlc Displacements in Sol1ds by Fast Electrons," U.S. AEC Report ORNL -4897 (1973).

[Om77] A. M. Omar, J. E. Robinson, and D. A. Thompson, J. Nuc1. Mater. 64, 121 (1977).

[0z73] O. Ozer and D. Garber, ENDF/B Sumary Documentation, Brookhaven National Laboratory Report BNL-17541 (1973).

[Pa62] V. K. Paré and D. O. Thompson, Acta Metal1. 10, 382 (1962).

[Pa73] V. K. Paré and 11. D. Guberman, J. App1. Phys. 44, 32 (1973).

[Pa74] D. M. Parkin and A. N. Goland, Brookhaven Nat1onal Laboratory Report BN-50434 (1974).

[Pa74a] V. K. Paré, H. D. Guberman, and P. B. McNee, J. App I. Phys. 45, 1615 (1974).

[Pa76] D. M. Parkin and A. N. Goland, Radiat. Eff. 28, 31 (1976).

[Ro70] M. T. Robinson, in Nuclear Fusion Reactors (British Nuclear Energy Society, London, 1970), p. 364 .

[Ro71] M. I. Robinson, Froc. Intern. Conf. Radiation Induced Voids in Metals, Albany, 1971, p. 397.

[Ro74] N. T. Robinson and I. M. Torrens, Phys. Rev. B 9, 5008 (1974).

[Ro75] M. T. Robinson, Proc. Intern. Conf. Fundamental Aspects of Radiation Damage in Metals, Gatlinburg, 1975, v. I. p. 1. 
[Ro75a] J. E. Roberto, J. Narayen, end M. J. Saltmarsh, Proc. Intern. Conf. Rad1ation Effects and Trit1um Technolagy for Fusion Reactore, Gatlinburg, 1975, v. II, P. 159.

[Ro76] J. B. Roberto and M. T. Robinson, J. Nucl. Mater. 61, 149 (1976).

[Ro77] J. B. Roberto, C. E. Klabunde, J. M. W1111ams, R. R. Coltman, Jr., M. J. Saltmarsh, and C. B. Fulmer, Appl. Phys. Lett. 30, 509 (1977).

[Ru67] M. Rühle and M. Wilkens, Philos. Mag. 15, 1075 (1967).

[Ru70] M. Rühle, F. Haüserman, and M. Rapp, Phys. Status Solid1 39, 609 (1970).

[Ru70a] M. Rühle, and J. C. Crump III, Fàys. Status Solid1 A 2 , 257 (1970).

[Sc69] W. Schilling, G. Burger, K. J sebeck, and H. Wenzl, in Vacancies and Interstitials in Metals (North-Holland, Amsterdam, 1969), p. 255.

[Sc70] W. Schilling, K. Schroeder, and H. Wollenberg, Phys. Status Solidi 38, 245 (1970).

[Si59] J. S1lccx and P. B. Hirsch, Philos. Mag. 4, 1356 (1959).

[Si67] P. Sigmund, G. P. Scheidler, and G. Roth, Proc. Intern. Conf. Solid State Physics Research with Accelerators, Upton, 1967, p. 374.

[Si71] H. M. Simpson, A. Sosin, G. R. Edwards, and S. L. Selffert, Phys. Rev. Lett. 26, 897 (1971).

[Si7la] P. Sigmund, M. T. Matthies, and D. L. Phillips, Radiat. Eff. 11, 39 (1971).

[Si72] E. x. Simpson and A. Sosin, Phys. Rev. B $\underline{5}, 1382$ (1972).

[Si72a] E. N. Simpson, A. Scsin, and D. F. Johnson, Phys. Rev. B 5, $1393(1972)$.

[Si73] H. M. Simpson, A. Sosin, and D. F. Johnson, J. App1. Phys. 44. 1435 (1973). 
[S174] H. M. Simpson and S. J. Kerkhoff, Phys. Rev. Lett. 33, 155 (1974).

[S176] H. M. Simpson and S. J. Kerkhoff, Radiat. Eff. 27, 191, 197 (1976).

[S177] H. M. Simpson and A. Sosin, Rev. Sc1. Instrum. -48, 1392 (1977).

[S178] H. M. Simpson, H. M. Hanson, and J. W. DeFord, Wright State Iniversity, unpublished, 1978.

[So68] A. Sosin and D. W. Keefer, in Microplasticity (J. Wiley and Sons, New York, 1968), p. 159 .

[Ta69] S. Takamura, H. Maeta, and S. Okuda, J. Phys. Soc. Jap. 26, 1120 (1969).

[Th56] D. O. Thompson and D. K. Holmes, J. App1. Phys. 27, 713 (1956).

[Th60] D. O. Thompson and V. K. Paré, J. Appl. Phys. 31, 528 (1960).

[Th67] D. O. Thompson, O. Buck, R. S. Barnes, and H. B. Huntington, J. Appl. Phys. 38, 3051 (1967).

[Th67a] D. O. Thompson, O. Buck, H. B. Huntington, and R. S. Barnes, J. Appl. Phys. 38, 3057 (1967).

[Th69] L. E. Thomas, T. Schober, and R. W. Balluffi, Radiat. Eff. 1, 257 (1969).

[Th69a] L. E. Thomas, T. Schober, and R. W. Balluffi, Radiat. Eff. 1, 269 (1969).

[Th71] J. E. Tnomas, T. O. Baldwin, and P. A. Dederichs, Phys. Rev. B 4,1167 (1971).

[Th75] D. A. Thompson, J. E. Robinson, R. S. Walker, A. M. Omar, and A. B. Campbel1, Proc. Intern. Conf. Radiation Effects and Tritium Technology for Fusion Reactors, Gatlinburg, 1975 , v. I., p. 382 .

[Va74] R. A. Van Konynenburg, Lawrence Livermore Laboratory Report UCRL-51393, Rev. 1 (1974). 


\section{APPENDIX}

\section{Calculation of the Neutron Flux}

Dosimetry folls were used to give a value of the integrated neutron flux for the experiments at the Tandem Van de Graaff. However, since the neutron source is not a point source (see Figure 28;, a simple $1 / \mathrm{r}^{2}$ law cannot be applied to compute the flux at the sample from the value measured at the foll. The following aspects of the calculation have to be considered:

(1) The incoming particle loses energy during its transit through the target. Thus the reaction cross section and outgoing neutron energy change along the length of the target.

(2) The angular distribution of the energy and intensity of neutrons from the reaction needs to be known at each position along the target.

(3) The beam is not infinitely narrow, so its width must be considered. Furthermore, the angular width of the dosimetry foil (or sample) is important. The foil and sample are placed coaxially with the beam axis.

(4) The neutron energy distribution at the foil differs slightly from that at the sample, due to geometrical effects.

To demonstrate the method of flux calculations, this procedure is outlined for run VDG-26 with $2 \mathrm{MeV}$ neutrons produced from the $H(t, n) H e^{3}$ reaction. The tritons for this reaction are accelerared with a machine energy of $5.01 \pm 0.01 \mathrm{MeV}$ down the 


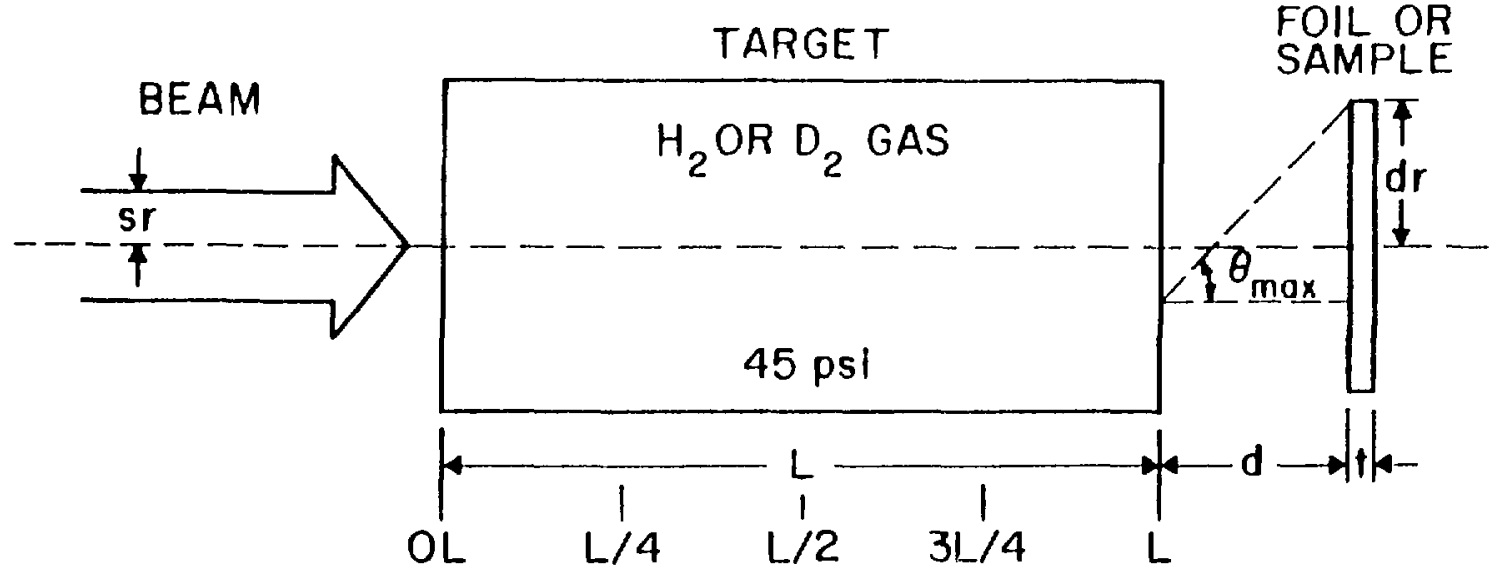

Figure 28: A schematic diagram of the geometrical parameters necessary for the calculation of neutron energy and flux at the sample position.

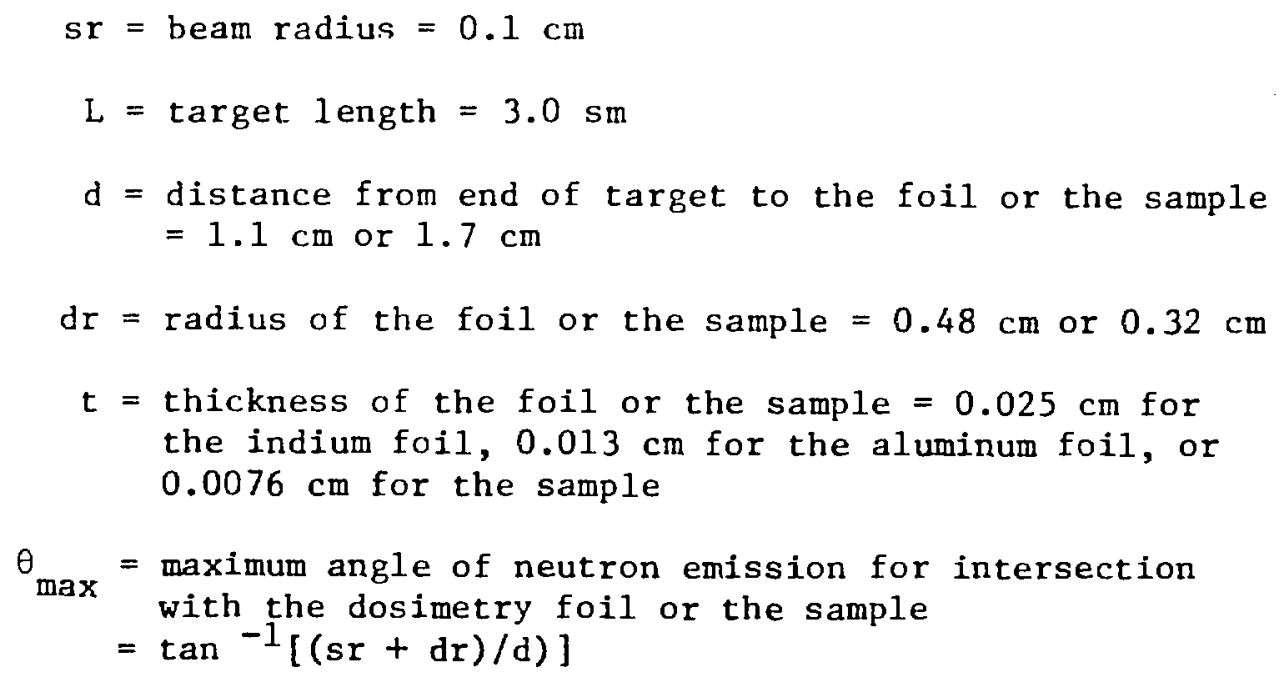


beam tube toward the target. A molybdenum foll separates the vacuum in the beam ine from the hydrogen gas in the target cell. The energy 1038 of the tritons in passing through the $5.3 \mathrm{mg} / \mathrm{cm}^{2}$ molybdenum foll is $0.42 \pm 0.04 \mathrm{MeV}$, giving an initial energy of the tritons in the gas cell of $4.59 \pm 0.04 \mathrm{MeV}$. Thus the variat1on in the machine energy and the energy lose in the Mo foll contributes a 17 error in the inftial energy of the tritons in the gas. At this point, the errors will be dropped; a discussion of all error sources will be given at the end. For hydrogen gas held at a pressure of $45 \mathrm{ps} 1$, the energy loss to excitation of the gas is $0.12 \mathrm{MeV} / \mathrm{cm}$ calculated using the Bethe stopping power formula. A program is available at the Van de Graaf to do this calculation. The slight energy dependence of the energy loss is ignored since little error is introduced by so doing in this particular example. The energies of the tritons at the beginning of the target (OL) and the positions $1 / 4,1 . / 2,3 \mathrm{~L} / 4$, and $L$ (the gold stop at the end of the gas cel1) are $4.59,4.50,4.41,4.32$, and 4.23 MeV, respectively.

Now we have the energy values of the tritons at five positions along the gas target. To calculate the flux and neutron energy spectrum, we need the reaction cross section and neutron energy for each triton energy. The energy of the outgoing neutron can be calculated from standard, relativistic kinematics. A set of tables has been compiled by Lisken and Paulsen (L173) which 
gives the cross sections for several neutron producing reactions. However, values for the Inverse reactions, $T(p, n) \mathrm{He}^{3}$, are 11 sted. To convert the inverse reaction cross sections to those for the forward reaction, the center-of-mass energies need to be computed for the same outgoing neutron energy. The following steps generate a table of $0^{\circ}$ differential cross sections, do/d $\left(0^{\circ}\right)$, and Legendre coefficients, $A_{1}$, necessary to calculate values of $d \sigma / d w\left(\theta^{\circ}\right)$ for the forward reaction.

(1) Find the proton energy which corresponds to each triton energy by equating the center-of-mass energies of the forward and inverse reactions. One finds that the proton energy is 0.334 times the triton energy, so for a triton of $4.59 \mathrm{MeV}$, the proton energy is $1.53 \mathrm{MeV}$.

(2) In the tables of Lisken and Paulsen find the values of $\mathrm{d} \sigma / \mathrm{d} \omega\left(0^{\circ}\right)(21.2 \mathrm{mb} / \mathrm{sr})$ and the Legendre coefficients (1.164, $-0.463,0.298$ ) for this proton energy. Interpolation may be necessary in some cases.

(3) The values of $\mathrm{d} \sigma / \mathrm{d} \omega\left(0^{\circ}\right)$ for the forward reaction is equal to $d \sigma / d \omega\left(180^{\circ}\right)$ for the inverse reaction:

$$
\begin{aligned}
\left.\frac{d \sigma}{d \omega}\left(0^{\circ}\right)\right|_{\text {for }} & =\left.\frac{d \sigma}{d \omega}\left(180^{\circ}\right)\right|_{\text {inv }}=\left.\frac{d \sigma}{d \omega}\left(0^{\circ}\right)\right|_{\text {inv }} \sum_{i=0}^{n} A_{i} P_{1}\left(180^{\circ}\right) \\
& =40.8 \mathrm{mb} / \mathrm{sr}
\end{aligned}
$$

where the $P_{i}$ are the Legendre polynomials and are equal to $(-1)^{i}$ for all $i$ at $\theta=180^{\circ}$. 
(4) Find the Legendre coefficients, $A_{1}^{\prime}$, for the forward reaction by:

$$
A_{1}^{\prime}=A_{1} / \sum_{1=0}^{n} A_{1} P_{1}
$$

so that $\sum_{1=0}^{n} A_{1}^{\prime}=1$. The individual values are $0.605,0.240$, and 0.155 for the triton energy of $4.59 \mathrm{MeV}$. For a reaction listed in the tables, the values of $\mathrm{dc} / \mathrm{d} \omega\left(0^{\circ}\right)$ and the Legendre coefficients can be directly read or interpolated. Now a complete set of neutron production cross sections and energies can be calculated for the $H(t, n)$ reaction.

A program called EDIT given by Lisken and Paulsen has been modified to compute the cross sections and energies at every degree to some maximum angle and punch output compatible with input to TIPI. The inputs to EDIT are rest masses of the reaction particles $\left(2808.44,938.79,939.58\right.$, and $2808.45 \mathrm{MeV} / \mathrm{c}^{2}$ for the triton, the hydrogen atom, the neutron and the helium atom, respectively), the Q-value of the reaction $(-0.764 \mathrm{MeV})$, the triton energies, and $d \sigma / d \omega\left(0^{\circ}\right)$ and the Legendre coefficients for each triton energy. The output of EDIT consists of five sets (one for each of the five triton energies) of angle and neutron energy pairs, the relative intensity of neutrons at $4^{\circ}$ intervals normalized to zero degrees, and the total reaction cross sections. For example, for the triton energy of $4.59 \mathrm{MeV}$, the neutron energies at $0^{\circ}$ and $30^{\circ}$ are 2.15 and $1.15 \mathrm{MeV}$, the anisotropy 
factor vartes from 1 at $0^{\circ}$ to 0.629 at $28^{\circ}$, and the total cross section $16310.6 \mathrm{mb}$.

TIPI uses these kinematics sets in conjunction with the geometrlc parameters describing the target and foll or sample (see Figure 28). The additional geometric parameters are the beam radius $(\mathrm{sr}=0.1 \mathrm{~cm})$, the target length $(\mathrm{L}=3 \mathrm{~cm})$, the foll or sample radius $(d r=0.48$ or $d r=0.32 \mathrm{~cm})$, the foll or sample thickness ( $t=0.025 \mathrm{~cm}$ for the indium foil or $t=0.0076 \mathrm{~cm}$ for the sample), and the distance from the end of the target to the foll or sample $(d=1.1$ or $d=1.7 \mathrm{~cm})$. A Monte Carlo technique is used to calculate the neutron energy spectra and the number of neutron hits. The program conserves time by limiting the possible neutron emission angle to the value given by the arctangent of the sum of the beam and foil radii divided by the target to foil distance $\left(\theta_{\max }=30^{\circ}\right)$. Neutrons emitted at angles greater than $30^{\circ}$ could not possibly hit the foil or sample. The number of hits for $10^{5}$ neutrons created is output along with a histogram of neutron energy versus relative intensity and the average neutron energy and its variance. This program is run for both the dosimetry foil and sample cases. The relative flux is found by dividing the number of hits $\mathrm{cm}^{-2}$ on the sample by the number of hits $\mathrm{cm}^{-2}$ on the foil. The number of hits, for this example, is 6630 and 22495 for the sample and foil respectively, so the relative flux at the sample is 0.663 . The average neutron energy at the foil is $1.8 \pm 0.2 \mathrm{MeV}$ and at the sample is $1.9 \pm 0.1 \mathrm{MeV}$. 
As staled in the text, the gamma rays from the decay of In ${ }^{115 m}$ are counted by the Radlochemistry Group. The raw counts are converted to a flux by using the mass and dlameter of the dosimetry foll, the half-11fe of decay $(4.5 \mathrm{hrs})$, the $\operatorname{In}\left(n, n^{\prime}\right)$ In $^{115 \mathrm{~m}}$ cross section at $1.8 \mathrm{MeV}(244 \mathrm{mb})$, the effictency of the garma detector, the time of counting after 1rradiation, the length of count time (40 or $60 \mathrm{~min}$ ), and the total irradiation time $(60 \mathrm{~min})$. Corrections are made for decay during irradiation and gamma counting. Using the relative flux found from TIPI (0.663), the flux computed at the dosimetry foil positton $\left(1.8 \times 10^{8} \mathrm{~cm}^{-2} \mathrm{~s}^{-1}\right)$ is converted to the flux at the sample position $\left(1.2 \times 10^{8} \mathrm{~cm}^{-2} \mathrm{~s}^{-1}\right)$.

The uncertainties in the distances of the foil or sample from the end of the target cell introduce the largest error into these calculations. Some idea of the magnitude of this exror can be estimated through the use of the TIPI program by altering the distances of the foil by $\pm 0.05 \mathrm{~cm}$ and the sample by $\pm 0.05 \mathrm{~cm}$ independently. Running TIPI for the various spacings, the change in the ratio of hits on the foil to hits on the sample can be computed. The error in the flux at the sample is roughly $9 \%$ due to the distance uncertainties. Additional uncertainties that contribute to the total flux error are the scattered neutrons striking the sample (a $2 \%$ effect), the angular span of the sample (2\%), the possibility of error in triton energy (1\%), and the 
error in the In $\left(n, n^{\prime}\right)$ In cross section $(3 \%)$. Inis leads to a total error of $10 \%$ in the sample flux.

At Pajarito Site, the Radiochemistry Group had calibrated the associated alpha partlcle counter by using aiuminum dosimetry folls. The source strength can be calculated using the total number of alpha particles counted, a conversion factor $\left(4.7 \times 10^{6}\right.$ $n / a)$, and the total irradiation time. Since the source is almost a point source from the view of the sample and is isutropic, a $1 / r^{2}$ rule can be used. The value of the flux calculated by this method compared to the value found by dosimetry foil techniques is in error by less than 3\%. Coupled with the distance uncertainty at lajarito site (9\%), again the flux error is $10 \%$ at the sample. The Mound Facility neutron genrrator has a 1.6 cro beatr spot and the sample was off-axis. Fortunately, by use of four copper pelicts as dosimeters, the position of the pellets and the sample relative to the beam axis couic be determined. By using the separate flux values for each pellet and the formula given by Van Konynenberg (Va74) for the flux at a point located off-axis, the flux at the sample could be computed. However, the reliability of the $f(u x$ is much less for these irradiations due to all the uncertainties in the distances measured and computed. The total error in this case is around $22 \%$.

\section{References}

[L173] H. Lisken and A. Paulsen, Nucl. Data Tables 11, 569 (1973).

[Va74] R. A. Van Konynenberg, Lawrence LIvermore Laboratory Report UCRL-51393, Rev. 1 (1974). 